\title{
Highly Stable Zr(IV)-Based Metal-Organic Frameworks with Chiral Phosphoric Acids for Catalytic Asymmetric Tandem Reactions
}

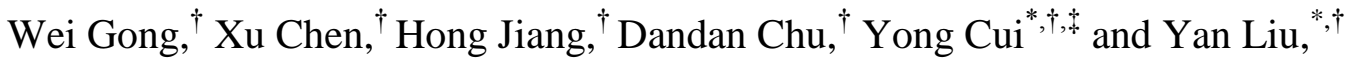 \\ ${ }^{\dagger}$ School of Chemistry and Chemical Engineering and State Key Laboratory of Metal Matrix \\ Composites, Shanghai Jiao Tong University \\ ${ }^{\ddagger}$ Collaborative Innovation Center of Chemical Science and Engineering, Tianjin 300072, China \\ Email: yongcui@sjtu.edu.cn; liuy@sjtu.edu.cn.
}

\section{Table of Content}

1. Materials and general procedures

2. Synthesis

3. General procedure for catalysis

4. Table S1. Crystal data and structure refinement

5. Table S2. Selected bond lengths $[\AA]$ and angles $\left[{ }^{\circ}\right]$ for Spiro-1

6. Table S3. Selected bond lengths $[\AA]$ and angles $\left[^{\circ}\right]$ for Spiro-2

7. Figures S1-S3. Additional X-ray crystallographic structures

8. Figure S4. CD spectra

9. Figure S5. TGA curves

10. Figure S6. IR spectra

11. Figure S7. Single crystal diffraction study of Spiro-2 after different treatment

12. Figure S8. Molecular mechanics simulation of large substrate

13. Table S4. Dye inclusion experiments

14. Figures S9-S12 and Tables S5-S11. Additional catalytic results

15. ${ }^{31} \mathrm{P}$ NMR spectra of the mixture of $\mathrm{ZrOCl}_{2}$ and $\mathrm{Me}_{4} \mathbf{L}_{\mathbf{1}}$

16. Table S12. Comparison of $\mathrm{S}_{\mathrm{BET}}$ and total solvent-accessible volume

17. Table S13. Hammett indicators and acidity measured by Hammett indicator tests

18. Figure S13. Kinetic curve of acetalization reaction

19. Figure S14. Imine intermediate detected in heterogeneous acetalization reaction

20. HPLC and NMR

21. References 


\section{Materials and General Procedures.}

All the chemicals are commercial available, and used without further purification. Elemental analyses of $\mathrm{C}$ and $\mathrm{H}$ were performed with an EA1110 CHNS-0 CE elemental analyzer. The IR (KBr pellet) spectra were recorded $\left(400-4000 \mathrm{~cm}^{-1}\right.$ region) on a Nicolet Magna 750 FT-IR spectrometer. The CD spectra were recorded on a J-800 spectropolarimeter (Jasco, Japan). Thermogravimetric analyses (TGA) were carried out in an $\mathrm{N}_{2}$ atmosphere with a heating rate of $10{ }^{\circ} \mathrm{C} / \mathrm{min}$ on a STA449C integration thermal analyzer. Powder X-ray diffraction (PXRD) data were collected on a Bruker D8 Advance diffractometer using $\mathrm{Cu} \mathrm{Ka}$ radiation. The calculated PXRD patterns were produced using the SHELXTL-XPOW program and single crystal reflection data. ${ }^{1} \mathrm{H}$ NMR,${ }^{13} \mathrm{C}$ NMR and ${ }^{31} \mathrm{P}$ NMR experiments were carried out on a MERCURY plus 400 spectrometer operating at resonance frequencies of $400 \mathrm{MHz}$. ESI-MS was recorded on a Finnigan LCQ mass spectrometer using dichloromethane-methanol as mobile phase. Analytical high performance liquid chromatography (HPLC) was performed on a Shimadzu 2010A with UV detection. Analytical CHIRALCEL OD-H and AD-H columns $(4.6 \mathrm{~mm} \times 25 \mathrm{~cm})$ from Daicel were used. $\mathrm{N}_{2}$ and $\mathrm{CO}_{2}$ adsorption isotherms were measured using a Micrometritics ASAP 2020 surface area analyzer at $77 \mathrm{~K}$ and $273 \mathrm{~K}$, respectively. Before the adsorption measurement, all the samples were exchanged with acetone for 3 times ( $6 \mathrm{~h}$ for each time) and then degassed on ASAP 2020 for $10 \mathrm{~h}$ at $100{ }^{\circ} \mathrm{C}$.

X-ray Crystallography. Single-crystal XRD data for Spiro-1 and Spiro-2 were collected on a Bruker SMART Apex II CCD-based X-ray diffractometer with $\mathrm{Cu}-\mathrm{K} \alpha$ radiation $(\lambda=1.54178 \AA)$ in the Instrumental Analysis Center of Shanghai Jiao Tong University, China and Beamline BL17B of National Facility for Protein Science at Shanghai Synchrotron Radiation Facility (SSRF) at $173 \mathrm{~K}$, respectively. The empirical absorption correction was applied by using the SADABS program (G. M. Sheldrick, SADABS, program for empirical absorption correction of area detector data; University of Götingen, Götingen, Germany, 1996). The structures were solved using direct method, and refined by full-matrix least-squares on $F^{2}$ by the SHELXTL-2014 software package. All non-H atoms were refined anisotropically except O28 in Spiro-1. DFIX, SADI, FLAT, DANG, EADP and SIMU restrains were used to obtain reasonable parameters due to the poor quality of crystal data. The solvent molecules were highly disordered, and attempts to locate and refine the solvent peaks were unsuccessful. Contributions to scattering due to these solvent molecules were removed using the SQUEEZE routine of PLATON, structures were then refined again using the data generated under OLEX2-1.2 (Dolomanov, O. V.; Bourhis, L. J.; Gildea, R. J.; Howard, J. A. K.; Puschmann, H. J. Appl.Crystallogr. 2009, 42, 339-341), The contents of the solvent region are not represented in the unit cell contents in the crystal data. Crystal data and details of the data collection are given in Table S1, while the selected bond distances and angles are presented in Table S2 and Table S3. CCDC 1871750 (Spiro-1) and 1871749 (Spiro-2) contain the supplementary crystallographic data for this paper. The data can be obtained free of charge from The Cambridge Crystallographic Data Centre via www.ccdc.cam.ac.uk/data_request/cif. 


\section{Synthesis}

\subsection{Synthesis of $(S)-\mathrm{H}_{4} \mathrm{~L}_{1}$}

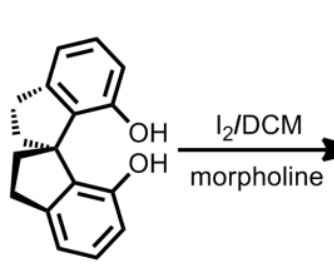

$(S)-1$

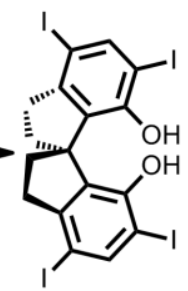

$(S)-2$

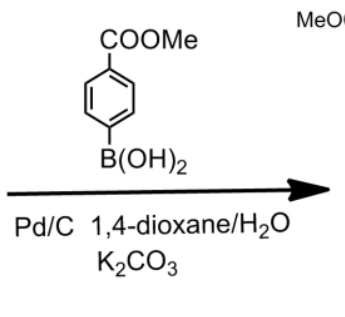

$\mathrm{MeOOC}$

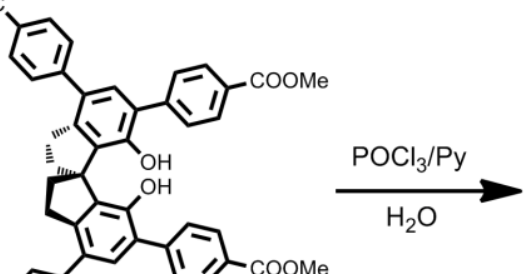

$(S)-3$

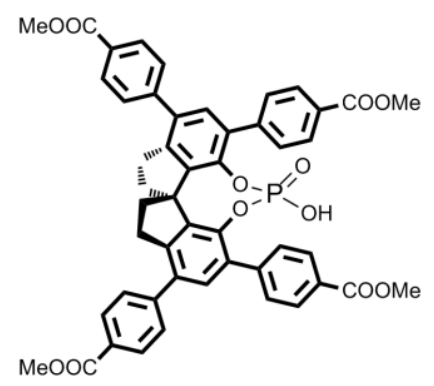

$(S)-\mathrm{Me}_{4} \mathrm{~L}_{1}$

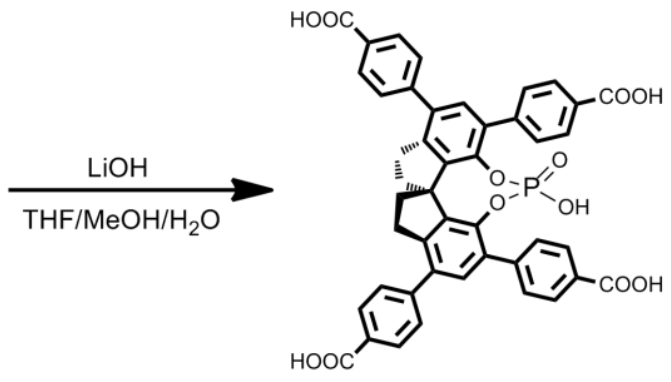

(S)- $\mathrm{H}_{4} \mathrm{~L}_{1}$

Synthesis of $(\boldsymbol{S})$-2. $(S)$-1 was synthesized according to the reported literature. ${ }^{[1,2]}$ To a solution of $(S)-1(2 \mathrm{~g}, 7.93 \mathrm{mmol})$ in DCM $(250 \mathrm{~mL})$ was added morpholine $(8.98 \mathrm{~g}, 103 \mathrm{mmol})$ and $\mathrm{I}_{2}(14.1$ $\mathrm{g}, 55.51 \mathrm{mmol})$. The mixture was stirred at room temperature for $12 \mathrm{~h}$. After that, $250 \mathrm{~mL}$ water was added. The organic phase was washed with diluent $\mathrm{HCl}$ and saturated $\mathrm{Na}_{2} \mathrm{~S}_{2} \mathrm{O}_{3}$, dried over anhydrous $\mathrm{Na}_{2} \mathrm{SO}_{4}$, and concentrated under reduced pressure. The resulted crude product was purified by column chromatography on silica gel (1:5, DCM-PE, v/v) to yield $(S)-2$ as a white solid (5.4 g, 90\%). ${ }^{1} \mathrm{H}$ NMR (400 MHz, $\left.\mathrm{CDCl}_{3}\right) \delta 7.85(\mathrm{~s}, 2 \mathrm{H}), 5.08(\mathrm{~s}, 2 \mathrm{H}), 3.03-2.88(\mathrm{~m}, 4 \mathrm{H}), 2.32$ $(\mathrm{dt}, J=12.8,9.2 \mathrm{~Hz}, 2 \mathrm{H}), 2.26-2.14(\mathrm{~m}, 2 \mathrm{H}) .{ }^{13} \mathrm{C} \mathrm{NMR}\left(101 \mathrm{MHz}, \mathrm{CDCl}_{3}\right) \delta: 151.43,150.53$, $144.41,133.75,84.82,83.49,63.69,37.07,36.71$.

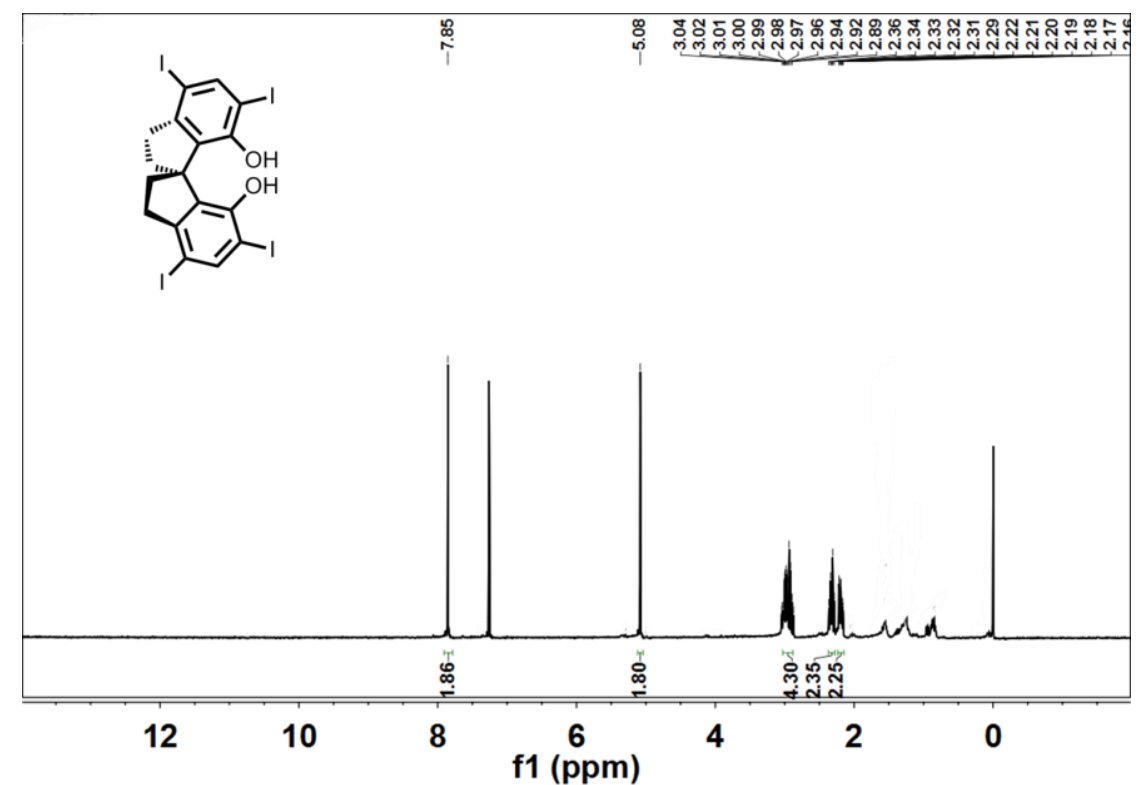




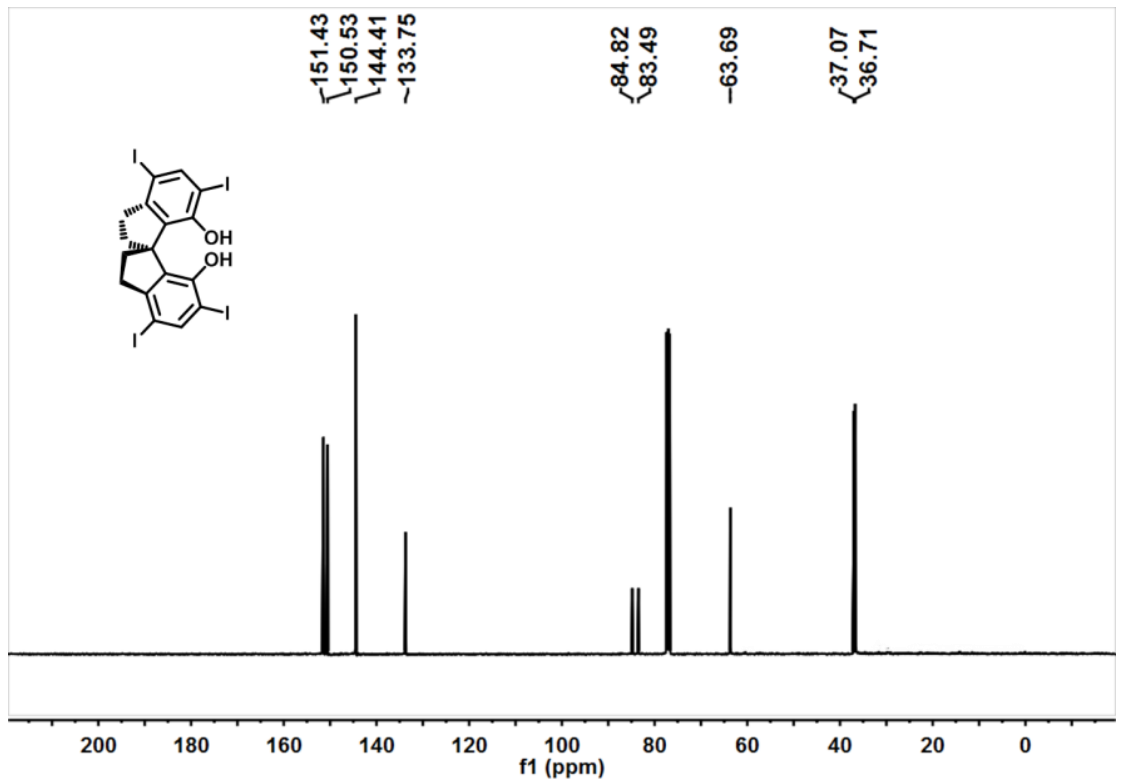

Synthesis of $(S)$-3. $(S)-2(2.5 \mathrm{~g}, 3.3 \mathrm{mmol}), 4$-(methoxycarbonyl)benzeneboronic acid (4.75 g, $26.4 \mathrm{mmol}), \mathrm{K}_{2} \mathrm{CO}_{3}(4.56 \mathrm{~g}, 33 \mathrm{mmol})$ and $10 \% \mathrm{Pd} / \mathrm{C}(0.7 \mathrm{~g}, 0.66 \mathrm{mmol})$ were added into a 250 $\mathrm{mL}$ flame-dried round-bottom flask. The flask was evacuated under vacuum and refilled with $\mathrm{N}_{2}$ for three times. Degassed dioxane $(40 \mathrm{~mL})$ and water $(40 \mathrm{~mL})$ were added, and the mixture was heated at $85{ }^{\circ} \mathrm{C}$ for $4 \mathrm{~h}$. Then the reaction mixture was cooled to room temperature and concentrated. The residue was extracted with DCM. The organic layer was collected, dried over anhydrous $\mathrm{Na}_{2} \mathrm{SO}_{4}$, and concentrated under reduced pressure. The crude product was purified by column chromatography on silica gel (3:1, PE-EA, v/v) to yield $(S)-3$ as a white solid (1.88 g, 75\%). ${ }^{1} \mathrm{H}$ NMR (400 MHz, $\left.\mathrm{CDCl}_{3}\right) \delta 8.09(\mathrm{dd}, J=12.6,8.1 \mathrm{~Hz}, 4 \mathrm{H}), 7.57(\mathrm{dd}, J=20.5,8.1 \mathrm{~Hz}$, $4 \mathrm{H}), 7.31(\mathrm{~s}, 1 \mathrm{H}), 5.18(\mathrm{~s}, 1 \mathrm{H}), 3.94(\mathrm{~s}, 3 \mathrm{H}), 3.91(\mathrm{~s}, 3 \mathrm{H}), 3.30-3.19(\mathrm{~m}, 1 \mathrm{H}), 3.10(\mathrm{dd}, J=16.5$, $7.9 \mathrm{~Hz}, 1 \mathrm{H}), 2.45(\mathrm{ddd}, J=32.1,17.8,10.0 \mathrm{~Hz}, 2 \mathrm{H}) .{ }^{13} \mathrm{C} \mathrm{NMR}(101 \mathrm{MHz}, \mathrm{CDCl} 3) \delta: 166.99$, $166.81,149.44,144.96,143.59,141.75,132.27,131.27,130.83,129.89,129.73,129.28,129.09$, $128.55,128.47,126.98,58.72,52.19,37.41,31.18$.

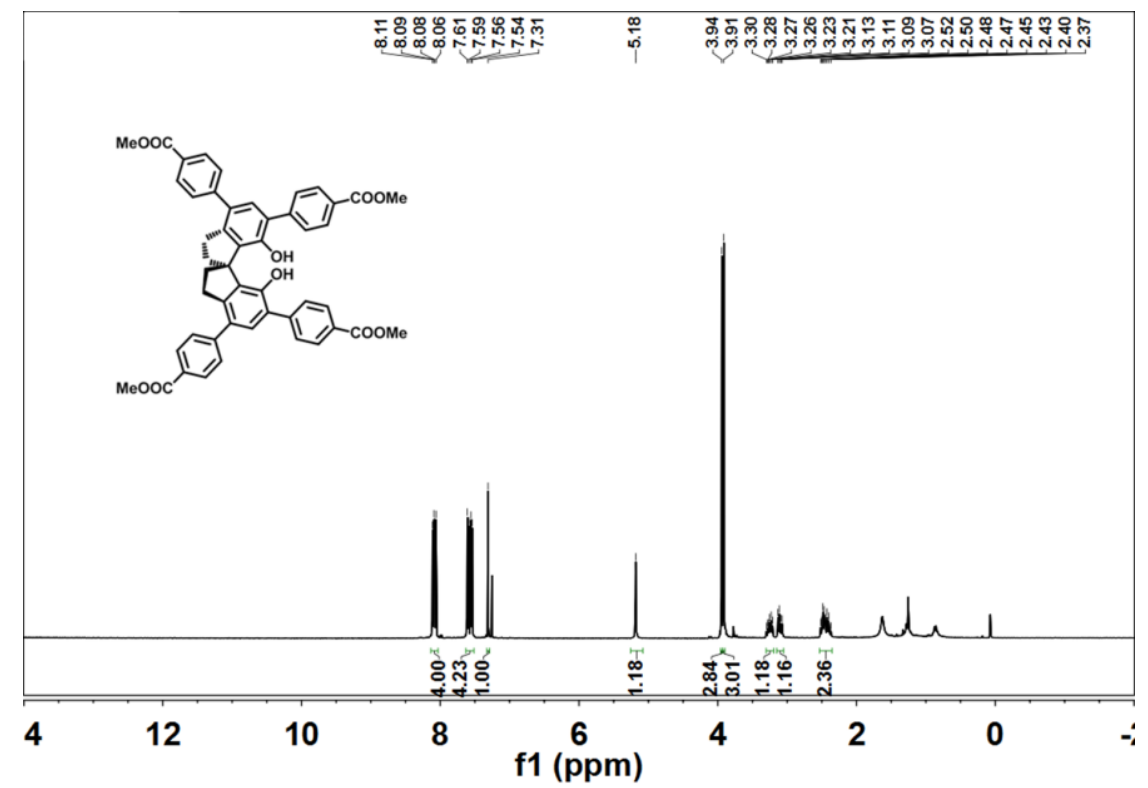




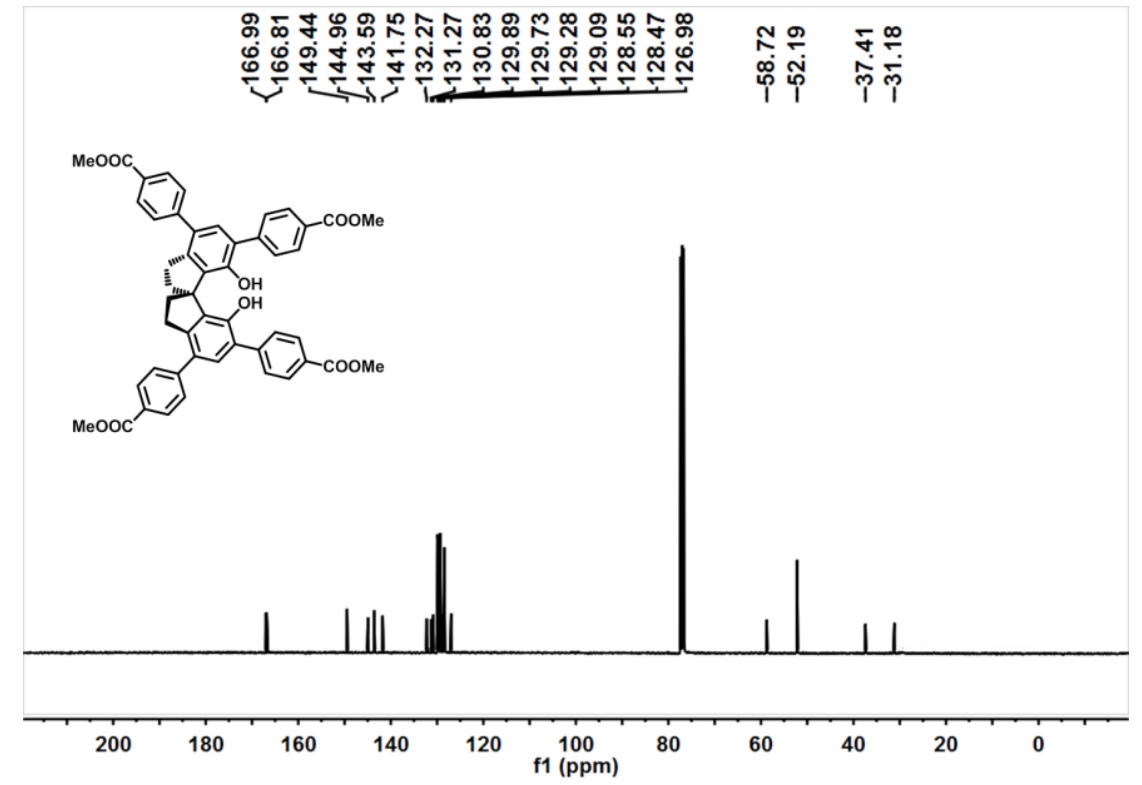

Synthesis of $(\boldsymbol{S})-\mathbf{M e}_{4} \mathbf{L}_{\mathbf{1}}$. To a solution of $(S)-3(1.88 \mathrm{~g}, 2.38 \mathrm{mmol})$ in anhydrous pyridine $(20 \mathrm{~mL})$ was added $\mathrm{POCl}_{3}(730 \mathrm{mg}, 4.76 \mathrm{mmol})$ slowly at $0{ }^{\circ} \mathrm{C}$. After stirring at $70{ }^{\circ} \mathrm{C}$ for $6 \mathrm{~h}$, the reaction mixture was quenched by addition of distilled water $(5 \mathrm{~mL})$ slowly at $0{ }^{\circ} \mathrm{C}$ and then stirred at $110{ }^{\circ} \mathrm{C}$ for $12 \mathrm{~h}$. After cooling to room temperature, the mixture was acidified to $\mathrm{pH}=5$ with $6 \mathrm{M}$ $\mathrm{HCl}$ and then extracted with DCM. The organic layer was washed with brine, dried over $\mathrm{Na}_{2} \mathrm{SO}_{4}$, and concentrated. The crude product was purified by column chromatography on silica gel $(1: 10$, MeOH-DCM, v/v) to yield (S)-Me $\mathrm{Me}_{4} \mathbf{L}_{\mathbf{1}}$ as a white solid (1.83 g, 90\%). ${ }^{1} \mathrm{H}$ NMR (400 MHz, $\mathrm{CDCl}_{3}$ ) $\delta: 8.12(\mathrm{~d}, \mathrm{~J}=6.8 \mathrm{~Hz}, 4 \mathrm{H}), 7.88(\mathrm{~d}, \mathrm{~J}=7.8 \mathrm{~Hz}, 4 \mathrm{H}), 7.56(\mathrm{~d}, \mathrm{~J}=5.7 \mathrm{~Hz}, 9 \mathrm{H}), 7.34(\mathrm{~s}, 2 \mathrm{H}), 3.96(\mathrm{~s}$, 6H), 3.75 (s, 6H), 3.24 (dd, J = 14.6, $9.5 \mathrm{~Hz}, 2 \mathrm{H}), 2.91(\mathrm{dd}, \mathrm{J}=14.6,6.8 \mathrm{~Hz}, 2 \mathrm{H}), 2.46$ (dd, J = $10.9,4.0 \mathrm{~Hz}, 2 \mathrm{H}), 2.34-2.24(\mathrm{~m}, 2 \mathrm{H}) .{ }^{13} \mathrm{C} \mathrm{NMR}\left(101 \mathrm{MHz}, \mathrm{CDCl}_{3}\right) \delta: 167.35,166.92,144.42$, $143.87,143.59,142.52,142.25,141.35,141.07,135.57,134.47,130.52,129.70,129.54,129.02$, 128.57, 60.39, 52.26, 38.44, 30.26. ${ }^{31} \mathrm{P}$ NMR (162 MHz, $\left.\mathrm{CDCl}_{3}\right) \delta:-9.80$.

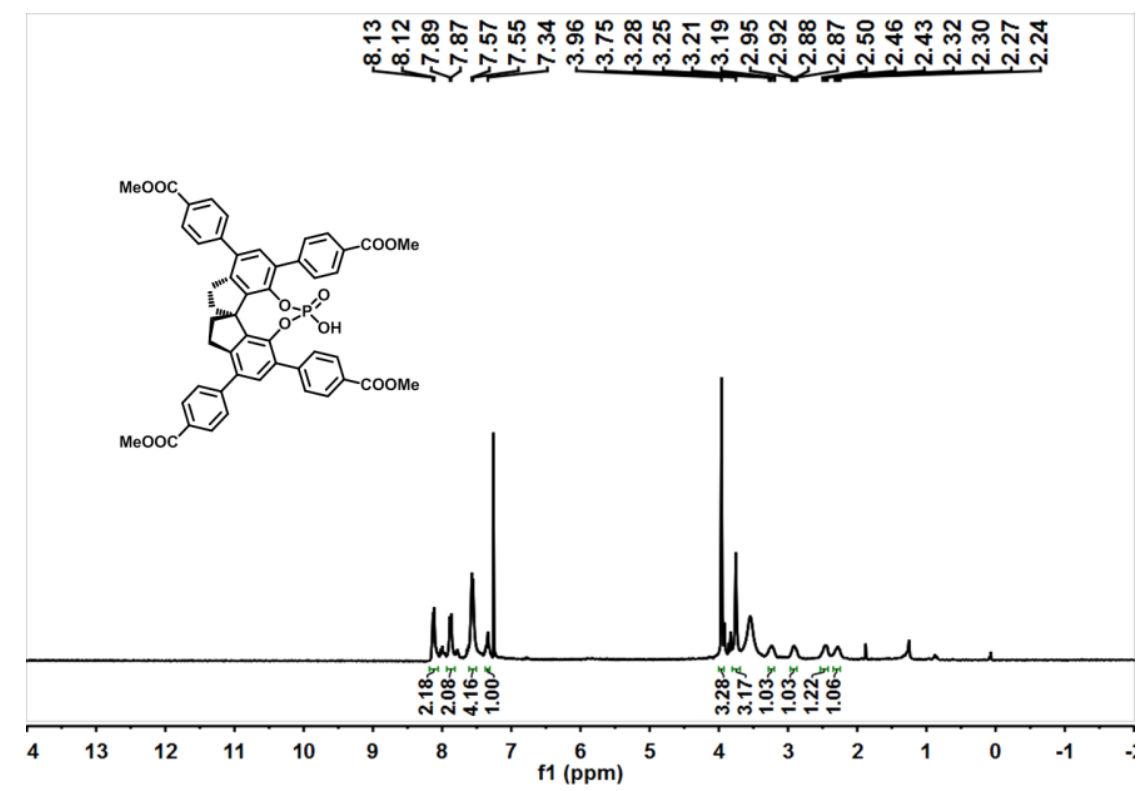



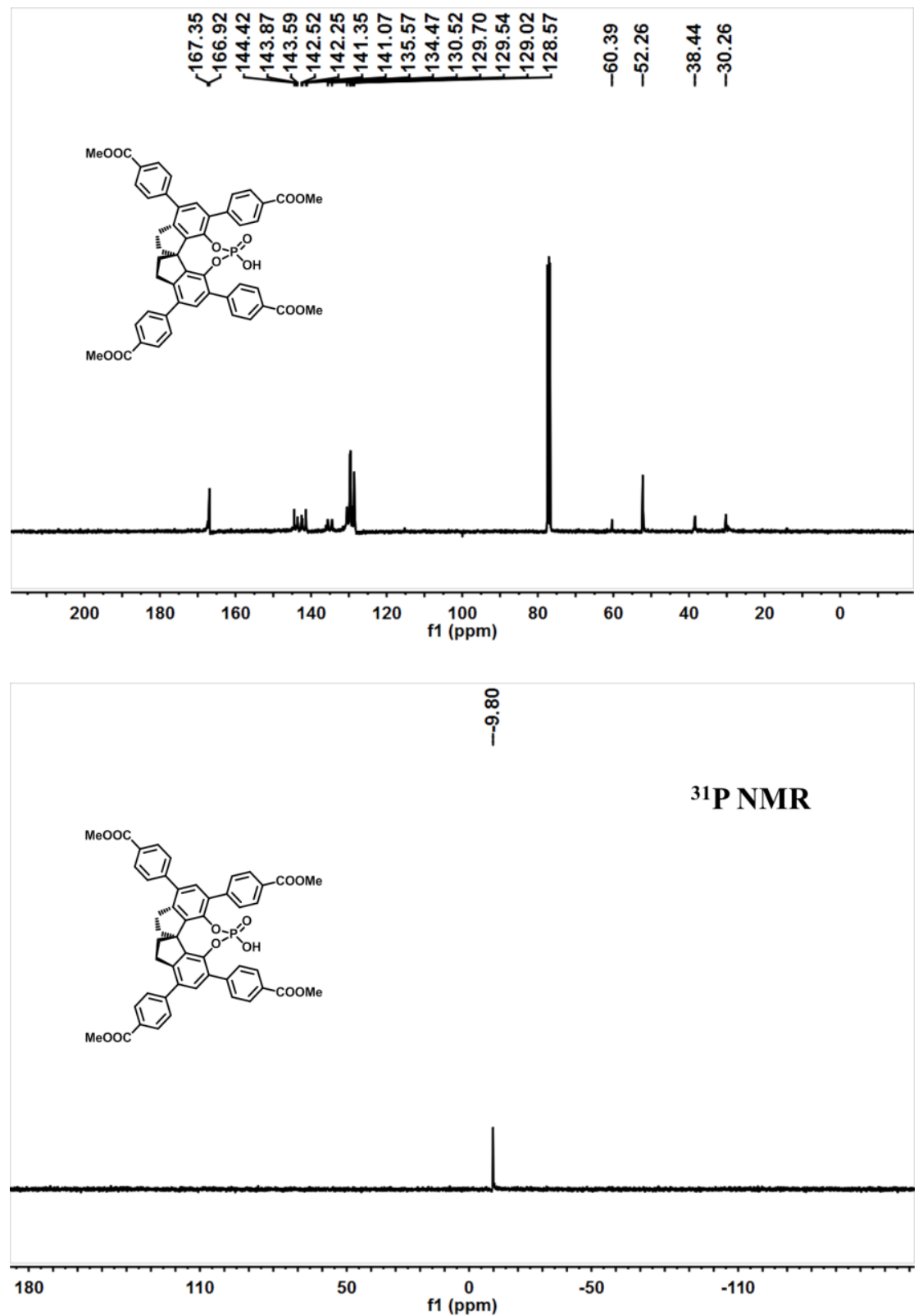

Synthesis of $(\boldsymbol{S})-\mathbf{H}_{\mathbf{4}} \mathbf{L}_{\mathbf{1}}$. A solution of $(S)-\mathrm{Me}_{4} \mathbf{L}_{\mathbf{1}}(1.83 \mathrm{~g}, 2.15 \mathrm{mmol})$ and $\mathrm{LiOH} \cdot \mathrm{H}_{2} \mathrm{O}(3.62 \mathrm{~g}, 86$ mmol) in THF $(20 \mathrm{~mL}), \mathrm{MeOH}(60 \mathrm{~mL})$ and $\mathrm{H}_{2} \mathrm{O}(60 \mathrm{~mL})$ was heated at $70{ }^{\circ} \mathrm{C}$ for $12 \mathrm{~h}$. After cooling to room temperature, the organic solvents were removed in vacuo and the water phase was acidified to $\mathrm{pH}=2$ with $3 \mathrm{M} \mathrm{HCl}$. The precipitate was filtrated and dried under vacuum at $60^{\circ} \mathrm{C}$ overnight. ( $S)-\mathrm{H}_{4} \mathbf{L}_{1}$ was obtained as a white solid $(1.63 \mathrm{~g}, 95 \%) .{ }^{1} \mathrm{H}$ NMR $\left(400 \mathrm{MHz}\right.$, DMSO-d $\left.\mathrm{d}_{6}\right)$ $\delta: 12.88(\mathrm{~s}, 4 \mathrm{H}), 8.02(\mathrm{~d}, \mathrm{~J}=8.2 \mathrm{~Hz}, 4 \mathrm{H}), 7.85(\mathrm{~d}, \mathrm{~J}=8.2 \mathrm{~Hz}, 4 \mathrm{H}), 7.72(\mathrm{t}, \mathrm{J}=7.0 \mathrm{~Hz}, 8 \mathrm{H}), 7.28(\mathrm{~s}$, 2H), $2.77(\mathrm{dd}, \mathrm{J}=16.2,8.2 \mathrm{~Hz}, 4 \mathrm{H}), 2.41(\mathrm{dd}, \mathrm{J}=10.9,5.9 \mathrm{~Hz}, 2 \mathrm{H}), 2.02(\mathrm{dd}, \mathrm{J}=20.0,9.9 \mathrm{~Hz}$, 2H). ${ }^{13} \mathrm{C}$ NMR (101 MHz, DMSO-d 6 ) $\delta: 167.94,167.69,145.80,145.71,144.77,143.73,142.94$, $134.75,133.09,129.99,129.75,129.30,129.11,99.98,60.02,30.27 .{ }^{31} \mathrm{P}$ NMR $(162 \mathrm{MHz}$, DMSO-d $\left.{ }_{6}\right) \delta:-11.73$. ESI-MS: m/z $\left.792.8235\left(\text { Calcd m/z } 793.1475 \text { for }\left[\mathrm{H}_{4} \mathbf{L} 1-\mathrm{H}\right]\right]^{-}\right)$. FTIR $(\mathrm{KBr}$, $\mathrm{cm}^{-1}$ ): 3386 (s), 1697 (s), 1607 (s), 1396 (m), 1244 (s), 1179 (m), 1094 (m), 1021 (m), 979 (w), $856(\mathrm{~m}), 781(\mathrm{w}), 719(\mathrm{w}), 546(\mathrm{w})$. 

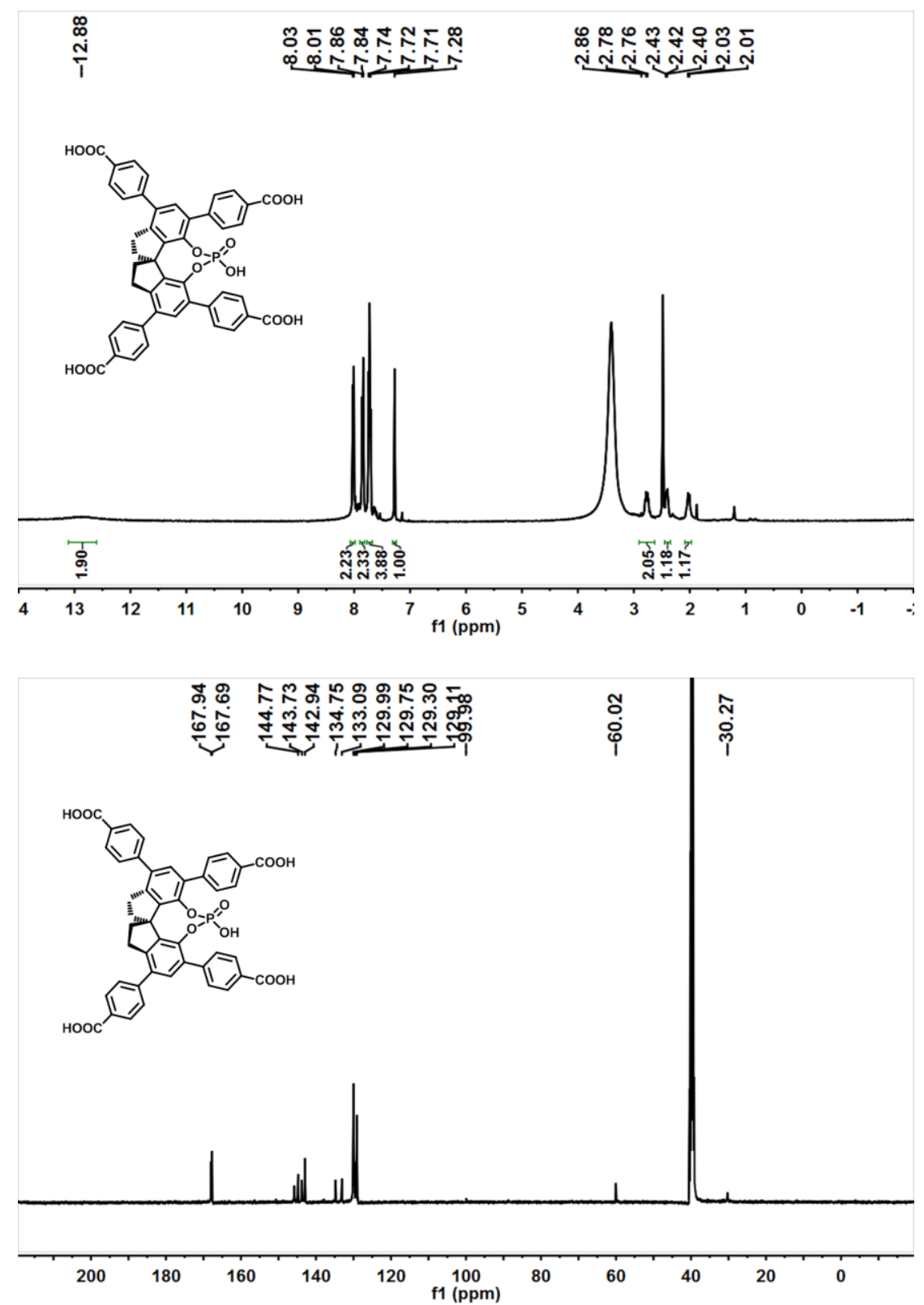


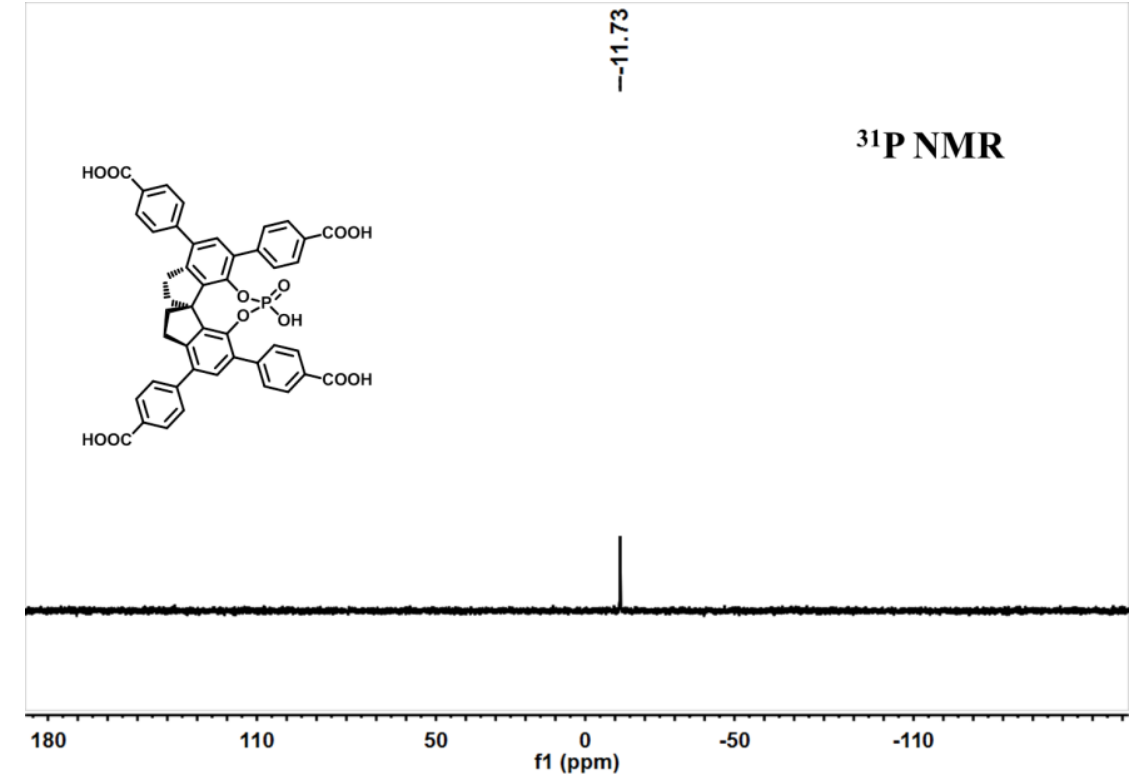

ESI-MS of $(S)-\mathrm{H}_{4} \mathrm{~L}_{1}$

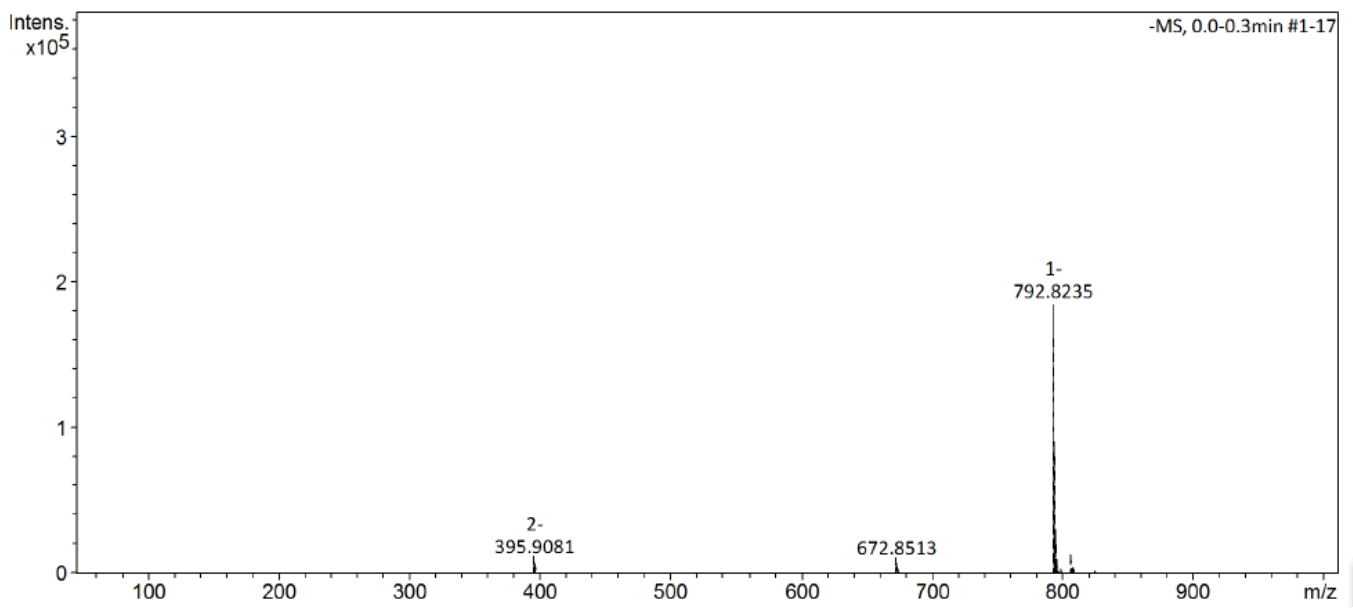




\subsection{Synthesis of $(S)-\mathrm{H}_{4} \mathrm{~L}_{2}$}

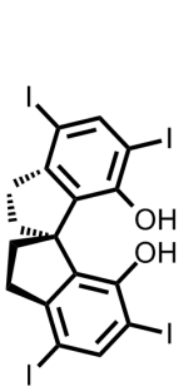

(S)-2
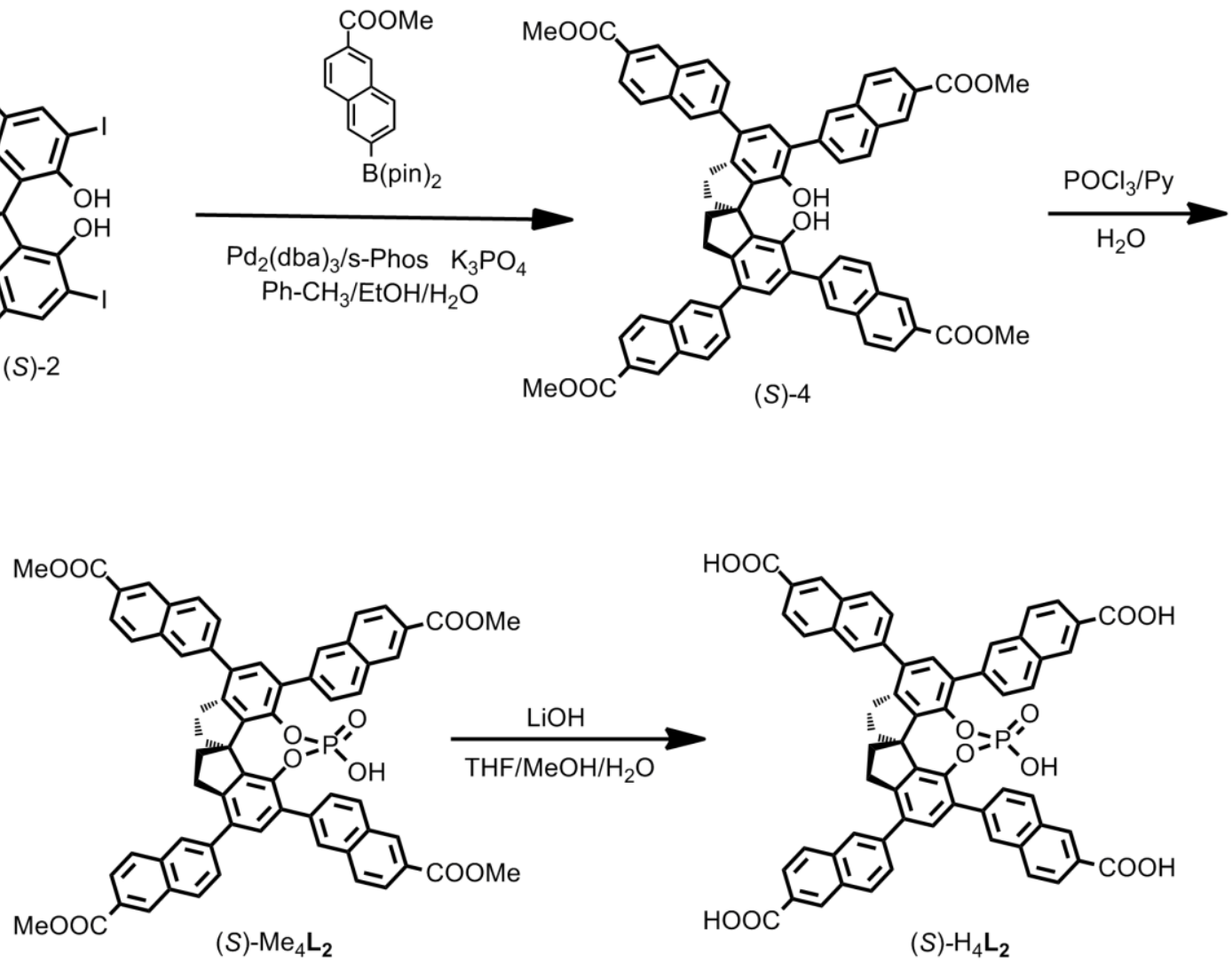

Synthesis of $(\boldsymbol{S})$-4. $(S)-2(3.0 \mathrm{~g}, 4.0 \mathrm{mmol}), 2$-(methoxycarbonyl)naphthalene-6-boronic acid pinacol ester $(7.5 \mathrm{~g}, 24 \mathrm{mmol}), \mathrm{K}_{3} \mathrm{PO}_{4}(6.8 \mathrm{~g}, 32 \mathrm{mmol}), \mathrm{Pd}_{2}(\mathrm{dba})_{3}(366 \mathrm{mg}, 0.4 \mathrm{mmol})$ and S-Phos ( $329 \mathrm{mg}, 0.8 \mathrm{mmol}$ ) were added into a $250 \mathrm{~mL}$ flame-dried round-bottom flask. The flask was evacuated under vacuum and refilled with $\mathrm{N}_{2}$ for three times. Degassed toluene $(60 \mathrm{~mL})$, ethanol $(20 \mathrm{~mL})$ and water $(20 \mathrm{~mL})$ were added, and the mixture was heated at $95{ }^{\circ} \mathrm{C}$ for $24 \mathrm{~h}$. Then the reaction mixture was cooled to room temperature and concentrated. The residue was extracted with DCM. The organic layer was collected, dried over anhydrous $\mathrm{Na}_{2} \mathrm{SO}_{4}$, and concentrated under reduced pressure. The crude product was purified by column chromatography on silica gel (2:1, PE-EA, v/v) to yield $(S)-4$ as a white solid (3.36 g, 85\%). ${ }^{1} \mathrm{H}$ NMR (400 MHz, $\left.\mathrm{CDCl}_{3}\right) \delta 8.64(\mathrm{~d}, J=18.9 \mathrm{~Hz}, 2 \mathrm{H}), 8.15-7.98(\mathrm{~m}, 6 \mathrm{H}), 7.96-7.85(\mathrm{~m}, 2 \mathrm{H}), 7.78$ (ddd, $J=16.2$, $8.5,1.5 \mathrm{~Hz}, 2 \mathrm{H}), 7.54(\mathrm{~s}, 1 \mathrm{H}), 5.50(\mathrm{~s}, 1 \mathrm{H}), 4.00(\mathrm{~d}, J=10.9 \mathrm{~Hz}, 6 \mathrm{H}), 3.48-3.18(\mathrm{~m}, 2 \mathrm{H}), 2.66-$ $2.52(\mathrm{~m}, 2 \mathrm{H}) .{ }^{13} \mathrm{C}$ NMR $\left(101 \mathrm{MHz}, \mathrm{CDCl}_{3}\right) \delta 167.24,167.16,149.51,143.57,140.43,137.17$, 135.59, 132.46, 131.79, 131.61, 131.39, 131.33, 130.86, 130.81, 129.66, 129.41, 128.36, 128.21, $127.86,127.82,127.63,127.47,127.39,127.00,125.79,125.73,75.04,58.93,53.41,52.26,24.85$, $22.69,14.11$. 

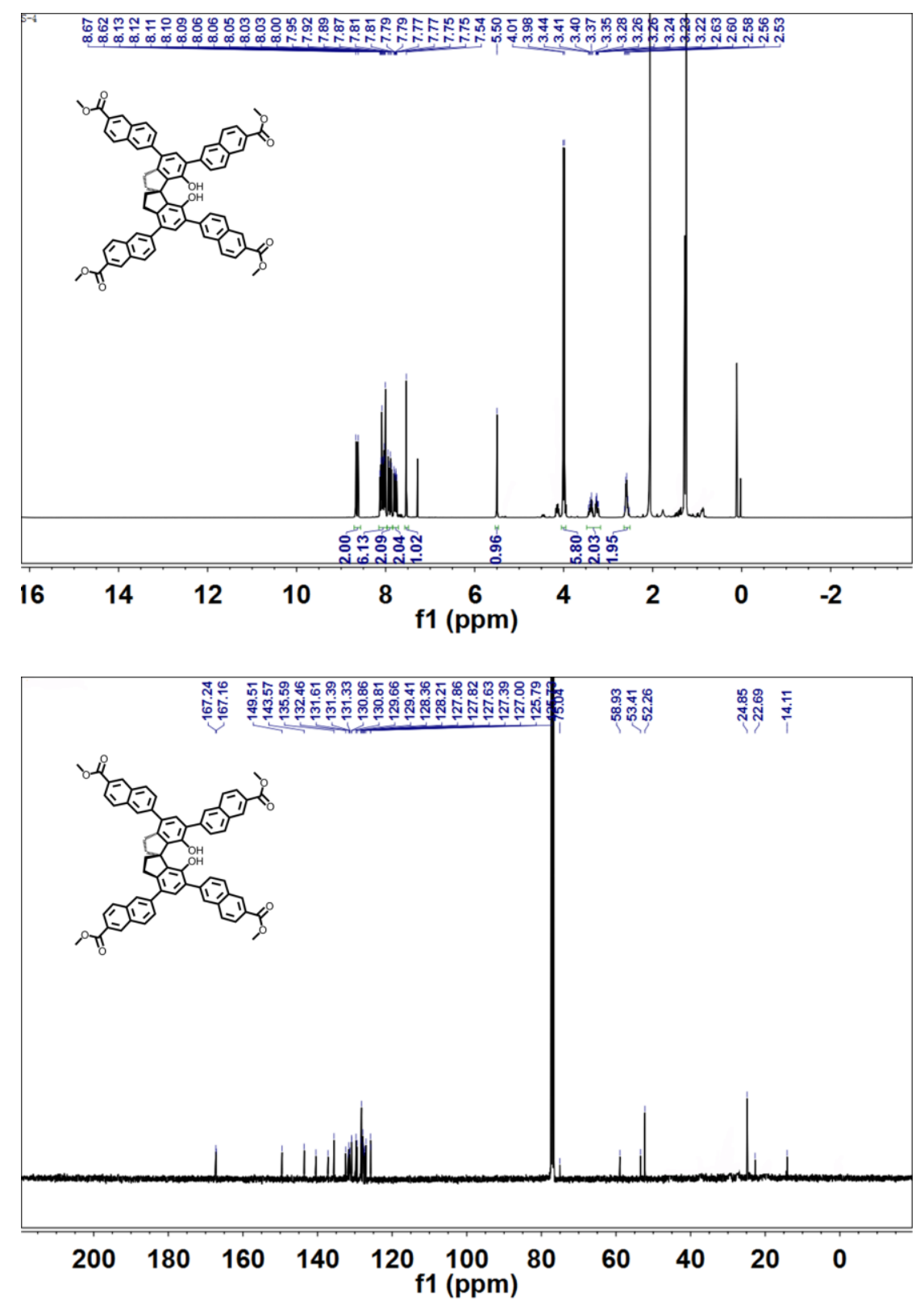

Synthesis of $(\boldsymbol{S})-\mathbf{M e}_{4} \mathbf{L}_{2}$. To a solution of $(S)-4(3.36 \mathrm{~g}, 3.4 \mathrm{mmol})$ in anhydrous pyridine (30 mL) was added $\mathrm{POCl}_{3}(1.1 \mathrm{~g}, 6.8 \mathrm{mmol})$ slowly at $0{ }^{\circ} \mathrm{C}$. After stirring at $80{ }^{\circ} \mathrm{C}$ for $10 \mathrm{~h}, 15 \mathrm{~mL}$ dioxane was added and then the reaction mixture was quenched by addition of distilled water $(8 \mathrm{~mL})$ slowly at $0{ }^{\circ} \mathrm{C}$ and then stirred at $110{ }^{\circ} \mathrm{C}$ for another $12 \mathrm{~h}$. After cooling to room temperature, the mixture was acidified to $\mathrm{pH}=5$ with $6 \mathrm{M} \mathrm{HCl}$ and then extracted with DCM. The organic layer was washed with brine, dried over $\mathrm{Na}_{2} \mathrm{SO}_{4}$, and concentrated. The crude product was purified by column chromatography on silica gel (1:10, MeOH-DCM, v/v) to yield (S)- $\mathrm{Me}_{4} \mathbf{L}_{\mathbf{2}}$ as a white solid (3.25 g, 91\%). ${ }^{1} \mathrm{H}$ NMR (400 MHz, $\left.\mathrm{CDCl}_{3}\right) \delta 9.10-7.31(\mathrm{~m}, 13 \mathrm{H}), 4.00(\mathrm{~s}, 3 \mathrm{H}), 3.69(\mathrm{~s}, 3 \mathrm{H})$, $3.30(\mathrm{~s}, 1 \mathrm{H}), 2.95(\mathrm{~s}, 1 \mathrm{H}), 2.50(\mathrm{~d}, J=42.6 \mathrm{~Hz}, 2 \mathrm{H}) .{ }^{13} \mathrm{C} \mathrm{NMR}\left(101 \mathrm{MHz}, \mathrm{CDCl}_{3}\right) \delta 167.11(\mathrm{~s})$, $145.30-142.07(\mathrm{~m}), 142.22(\mathrm{~s}), 141.14(\mathrm{~d}, J=216.2 \mathrm{~Hz}), 139.20(\mathrm{~s}), 139.20-137.53(\mathrm{~m})$, 135.43 (s), 135.19 (d, $J=22.8 \mathrm{~Hz}), 131.19$ (d, $J=14.2 \mathrm{~Hz}), 130.75$ (d, $J=19.5 \mathrm{~Hz}), 132.01$ $129.14(\mathrm{~m}), 130.42(\mathrm{~s}), 130.32-129.81(\mathrm{~m}), 128.81(\mathrm{~s}), 128.14$ (s), 127.59 (s), 126.67 (dd, $J=$ 95.8, $81.1 \mathrm{~Hz}), 125.16$ (s), 125.14 (s), 60.58 (s), 52.20 (d, $J=14.5 \mathrm{~Hz}), 38.94$ (s), 30.06 (d, $J=$ $57.3 \mathrm{~Hz}), 29.67-29.58(\mathrm{~m}), 29.32$ (s), 22.69 (s), 14.08 (s). ${ }^{31} \mathrm{P}$ NMR $\left(162 \mathrm{MHz}, \mathrm{CDCl}_{3}\right) \delta$ : 
$-10.54$
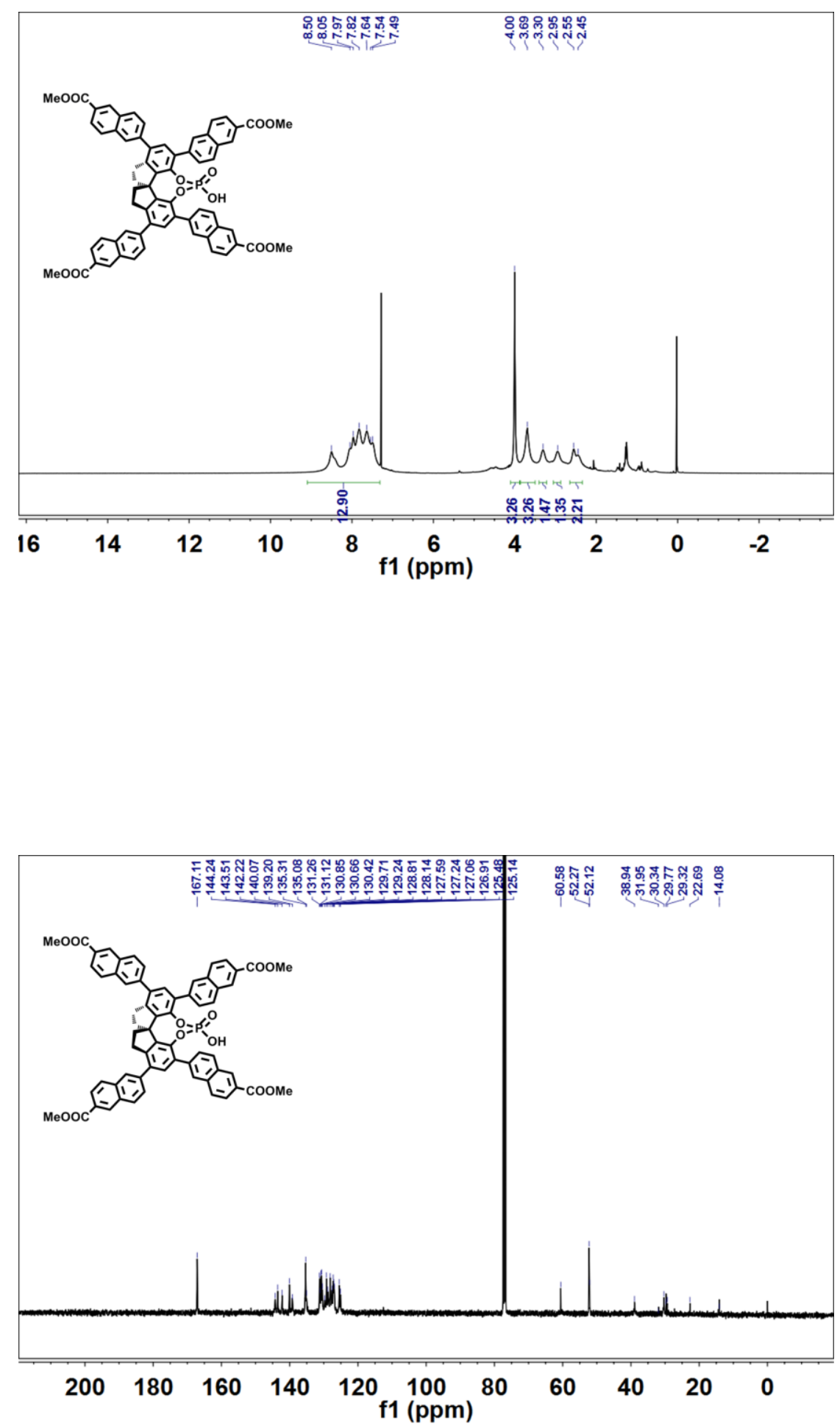


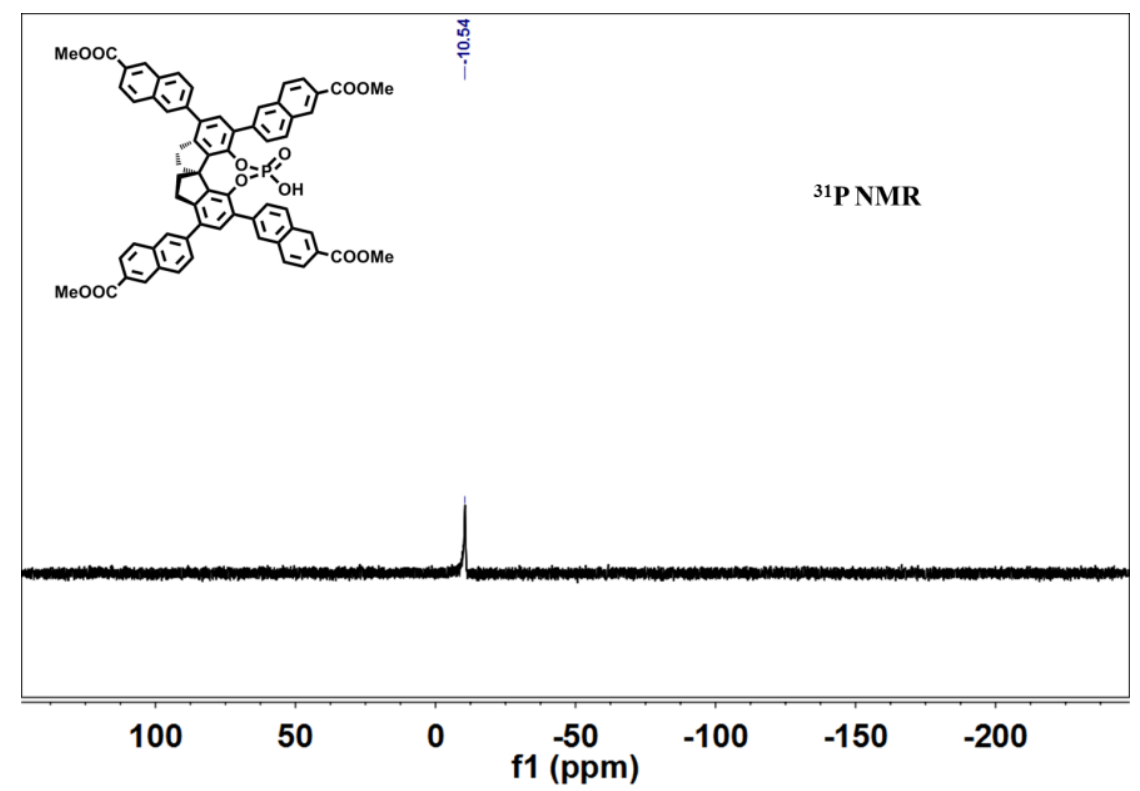

Synthesis of $(S)-\mathbf{H}_{4} \mathbf{L}_{2}$. A solution of $(S)-\mathrm{Me}_{4} \mathbf{L}_{2}(3.25 \mathrm{~g}, 3.09 \mathrm{mmol})$ and $\mathrm{LiOH} \cdot \mathrm{H}_{2} \mathrm{O}(4.95 \mathrm{~g}$, $123.6 \mathrm{mmol})$ in THF $(25 \mathrm{~mL}), \mathrm{MeOH}(75 \mathrm{~mL})$ and $\mathrm{H}_{2} \mathrm{O}(75 \mathrm{~mL})$ was heated at $70{ }^{\circ} \mathrm{C}$ for $12 \mathrm{~h}$. After cooling to room temperature, the organic solvents were removed in vacuo and the water phase was acidified to $\mathrm{pH}=2$ with $3 \mathrm{M} \mathrm{HCl}$. The precipitate was filtrated and dried under vacuum at $60^{\circ} \mathrm{C}$ overnight. (S) $-\mathrm{H}_{4} \mathbf{L}_{1}$ was obtained as a white solid $(2.92 \mathrm{~g}, 95 \%) .{ }^{1} \mathrm{H}$ NMR $(400 \mathrm{MHz}$, DMSO-d $\left.\mathrm{d}_{6}\right) \delta 8.63(\mathrm{~d}, J=24.9 \mathrm{~Hz}, 1 \mathrm{H}), 8.48(\mathrm{~d}, J=18.3 \mathrm{~Hz}, 1 \mathrm{H}), 8.43-8.16(\mathrm{~m}, 3 \mathrm{H}), 8.10(\mathrm{t}, J=$ $9.2 \mathrm{~Hz}, 1 \mathrm{H}), 8.08-7.87(\mathrm{~m}, 5 \mathrm{H}), 7.51(\mathrm{~s}, 1 \mathrm{H}), 3.50(\mathrm{~s}, 2 \mathrm{H}), 3.02-2.84(\mathrm{~m}, 1 \mathrm{H}), 2.17(\mathrm{~d}, J=8.4$ $\mathrm{Hz}, 1 \mathrm{H}) .{ }^{13} \mathrm{C}$ NMR $\left(101 \mathrm{MHz}, \mathrm{DMSO}-\mathrm{d}_{6}\right) \delta 172.51,168.26,146.40,146.32,143.10,143.08$, $142.70,140.40,139.63,135.47,135.31,135.21,133.29,131.53,131.34,130.57,130.39,129.79$, $129.69,129.37,128.72,128.63,128.58,128.25,128.21,127.39,126.17,125.45,60.13,30.29$, 21.61. ${ }^{31} \mathrm{P}$ NMR (162 MHz, DMSO) $\delta$-12.26. ESI-MS: m/z 1039.03 (Calcd m/z 1039.22 for $\left.\left[\mathrm{H}_{4} \mathbf{L 2}+2 \mathrm{Na}-\mathrm{H}\right]^{+}\right)$. FTIR $\left(\mathrm{KBr}, \mathrm{cm}^{-1}\right): 3401(\mathrm{~s}), 1696(\mathrm{~s}), 1628(\mathrm{~s}), 1459(\mathrm{~m}), 1396(\mathrm{~m}), 1219$ (s), 1090 (s), $1024(\mathrm{~m}), 998(\mathrm{w}), 914(\mathrm{~m}), 887(\mathrm{w}), 849(\mathrm{w}), 816(\mathrm{~m}), 546(\mathrm{w})$.

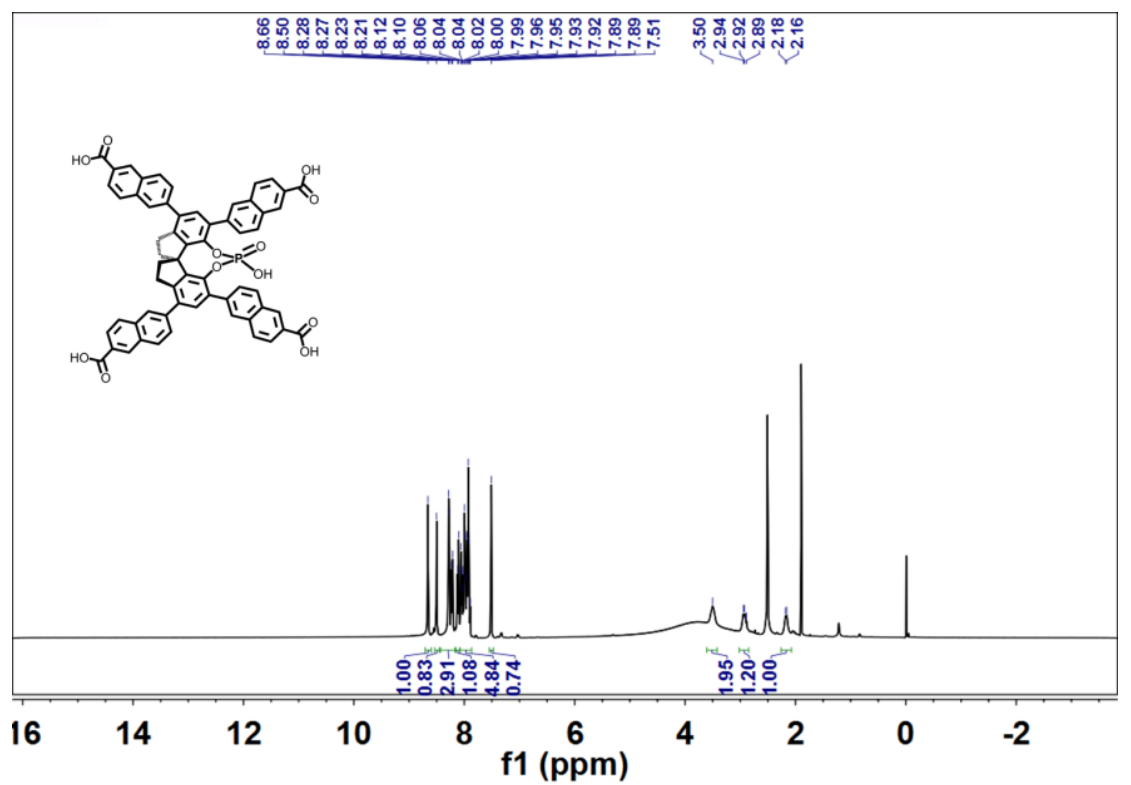



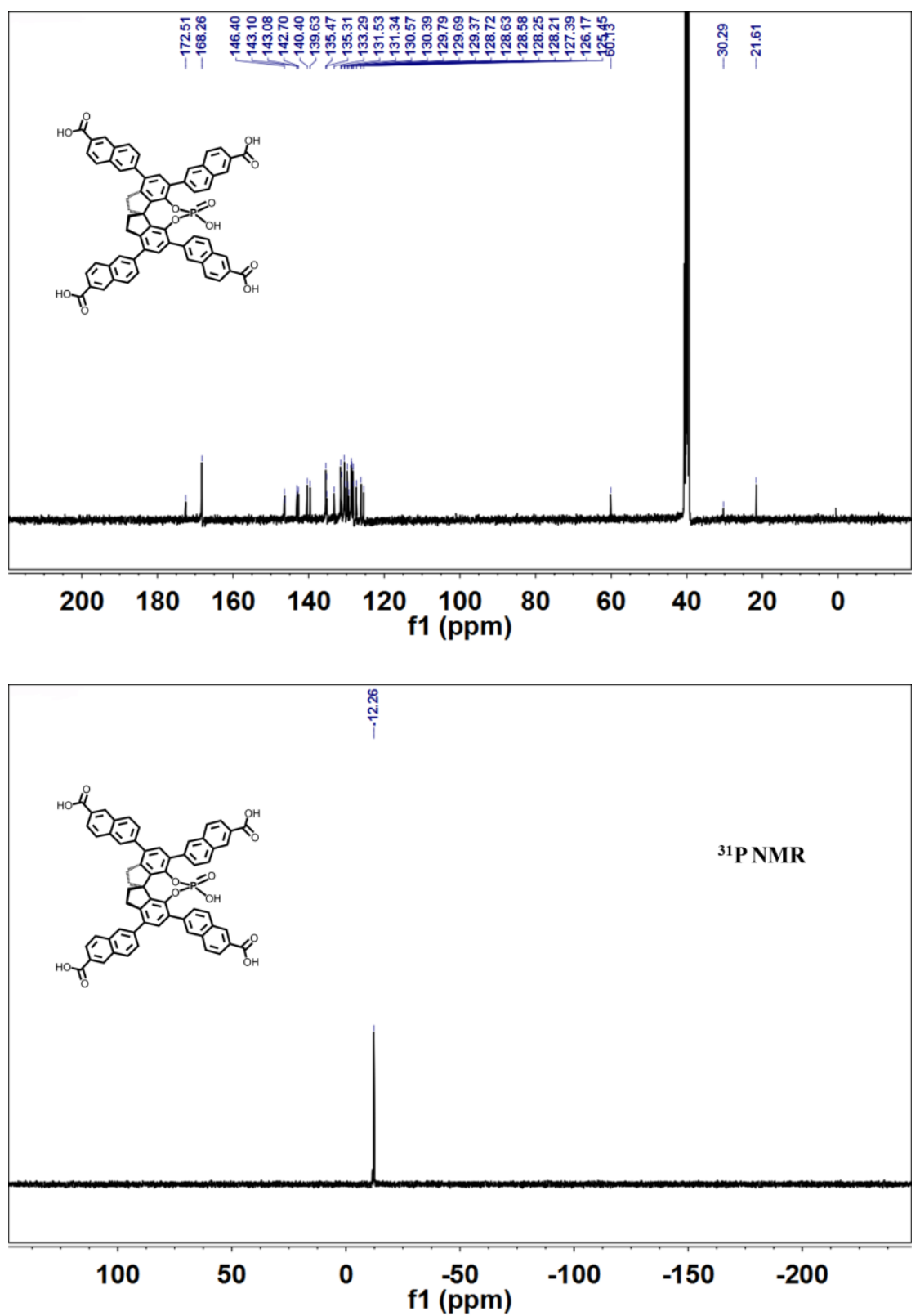

ESI-MS of $(S)-\mathrm{H}_{4} \mathrm{~L}_{2}$

CY20180509SAMPLE5 \#50 RT: 0.14 AV: 1 NL: $1.37 E 5$

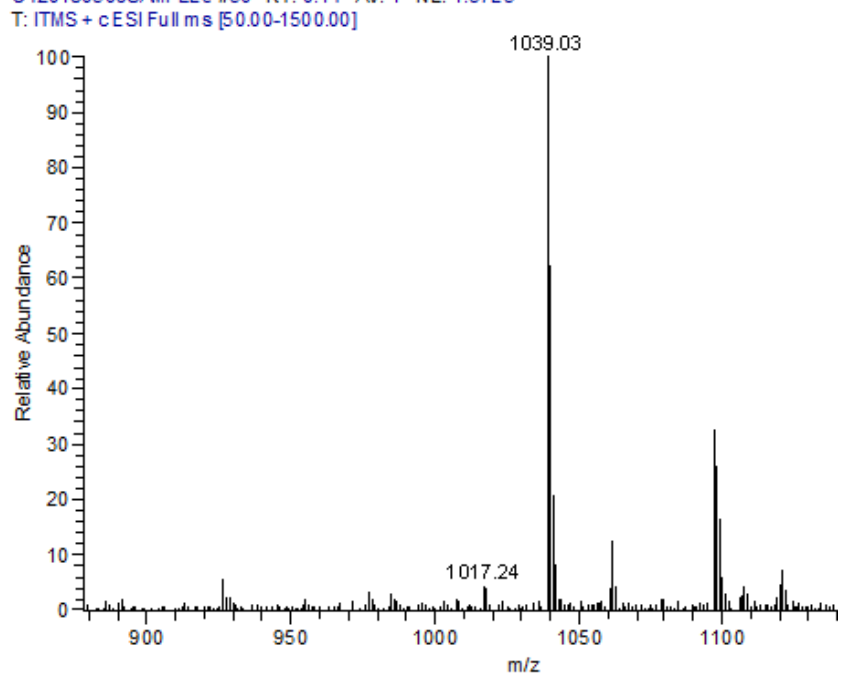




\subsection{Synthesis of Spiro-1 and Spiro-2}

Spiro-1. A mixture of $\mathrm{ZrCl}_{4}(52 \mathrm{mg}, 0.22 \mathrm{mmol})$ and $\mathrm{H}_{4} \mathbf{L}_{\mathbf{1}}(60 \mathrm{mg}, 0.075 \mathrm{mmol})$ was ultrasonic dissolved in DMF (12 mL). $5 \mathrm{~mL}$ anhydrous formic acid was added and the mixture was heated at $120{ }^{\circ} \mathrm{C}$ for 3 days. Colorless block crystals $(53 \mathrm{mg}, 48 \%$ ) were collected, washed with DMF and acetone, and dried in air. FTIR (KBr, cm ${ }^{-1}$ ): 3401 (m), 2925 (w), 1657 (s), 1605 (s), 1497 (s), 1414 (s), 1258 (w), 1193 (w), 1097 (m), 1023 (w), 836 (w), 789 (w), 658 (w), 556 (w), 495 (w). Elemental Analysis: Calcd for $\mathrm{C}_{135} \mathrm{H}_{78} \mathrm{Zr}_{9} \mathrm{O}_{60} \mathrm{P}_{3}$ : C, 45.33; H, 2.18; Found: C, 46.22; H, 1.98 .

Spiro-2. A mixture of $\mathrm{ZrCl}_{4}(28 \mathrm{mg}, 0.12 \mathrm{mmol})$ and $\mathrm{H}_{4} \mathbf{L}_{2}(30 \mathrm{mg}, 0.03 \mathrm{mmol})$ was ultrasonic dissolved in DMF (4 mL). $1.2 \mathrm{~mL}$ anhydrous formic acid was added and the mixture was heated at $120{ }^{\circ} \mathrm{C}$ for 3 days. Colorless block crystals ( $32 \mathrm{mg}, 62 \%$ ) were collected, washed with DMF and EtOH, and dried in air. FTIR (KBr, cm $\left.{ }^{-1}\right): 3396$ (m), 2935 (w), 1654 (s), 1597 (s), 1383 (s), 1259 (w), $1186(\mathrm{w}), 1095$ (m), 819 (m), 753 (w), 659 (s), 543 (w). Elemental Analysis: Calcd for $\mathrm{C}_{183}$ $\mathrm{H}_{102} \mathrm{Zr}_{9} \mathrm{O}_{60} \mathrm{P}_{3}: \mathrm{C}, 52.59$; H, 2.44; Found: C, 53.11; H, 2.23 .

\section{General procedure for catalysis}

Explanation: mol\% loading catalyst means catalyst loading based on the the substrate with lower molar equivalents. For example: when we use $0.1 \mathrm{mmol}$ substrate $\mathrm{A}$ and $0.15 \mathrm{mmol}$ substrate B for reaction, $1.4 \mathrm{~mol} \%$ catalyst means $1.4 \times 0.01 \times 0.1 \mathrm{mmol}$ (substrate A) $=1.4 \mu \mathrm{mol}$ $\mathrm{MOF}=1.4 \mu \mathrm{mol} * 2=2.8 \mu \mathrm{mol}$ active phosphoric acid sites. One mol of catalyst means one mol of the MOF or $2 \mathrm{~mol}$ of active phosphoric acid sites.

3.1 Asymmetric condensation/amine addition cascade sequence of 2-aminobenzamide with arylaldehydes

The catalysts Spiro-1 and Spiro-2 were exchanged with acetone thoroughly and then activated at $60{ }^{\circ} \mathrm{C}$ to remove the guest solvents.

To a flame-dried Schlenk Pressure Tube was added activated Spiro-1 (5 mg, $0.0014 \mathrm{mmol}$ ), 2-aminobenzamide $(0.1 \mathrm{mmol})$, anhydrous $\mathrm{MgSO}_{4}(100 \mathrm{mg})$ and $0.5 \mathrm{~mL}$ anhydrous $\mathrm{CHCl}_{3}$. The resulting mixture was stirred at $60{ }^{\circ} \mathrm{C}$ for $30 \mathrm{~min}$ and aldehydes $(0.15 \mathrm{mmol})$ in $0.5 \mathrm{~mL} \mathrm{CHCl}_{3}$ was added via syringe. The mixture was stirred at $60{ }^{\circ} \mathrm{C}$ until the reaction completed. Chromatography on silica gel $(1: 2$, EA-PE, v/v) afforded the desired products.

\subsection{Deacetalization/acetalization tandem reaction of 2 -aminobenzamide with aldehyde dimethylacetals}

The catalysts Spiro-1 and Spiro-2 were exchanged with acetone thoroughly and then activated at $220{ }^{\circ} \mathrm{C}$ to generate the Lewis acidic $\mathrm{Zr}(\mathrm{IV})$ sites.

To a flame-dried Schlenk Pressure Tube was added activated Spiro-1 (5 mg, $0.0014 \mathrm{mmol}$ ), 2-aminobenzamide $(0.1 \mathrm{mmol})$, anhydrous $\mathrm{MgSO}_{4}(100 \mathrm{mg})$ and aldehyde dimethylacetals $(0.15$ mmol) and $1 \mathrm{~mL}$ fresh $\mathrm{CHCl}_{3}$ (not anhydrous, trace water is needed). The resulting mixture was stirred at $60{ }^{\circ} \mathrm{C}$ until the reaction completed. Chromatography on silica gel $(1: 2$, EA-PE, v/v) afforded the desired products.

\subsection{Deacetalization/acetalization tandem reaction of 2-aminobenzamide with aldehyde cyclic acetals}

The catalysts Spiro-1 and Spiro-2 were exchanged with acetone thoroughly and then activated at $220{ }^{\circ} \mathrm{C}$ to generate the Lewis acidic $\mathrm{Zr}(\mathrm{IV})$ sites.

To a flame-dried Schlenk Pressure Tube was added activated Spiro-1 (5 mg, 0.0014 mmol), 
2-aminobenzamide $(0.1 \mathrm{mmol})$, anhydrous $\mathrm{MgSO}_{4}(100 \mathrm{mg})$ and aldehyde cyclic acetals $(0.15$ mmol) and $1 \mathrm{~mL}$ fresh $\mathrm{CHCl}_{3}$ (not anhydrous, trace water is needed). The resulting mixture was stirred at $60{ }^{\circ} \mathrm{C}$ until the reaction completed. Chromatography on silica gel (1:2, EA-PE, v/v) afforded the desired products.

\subsection{Asymmetric Friedel-Crafts reaction of $\mathrm{N}$-sulfonyl aldimines with indole}

The catalysts Spiro-1 and Spiro-2 were exchanged with acetone thoroughly and then activated at $60{ }^{\circ} \mathrm{C}$ to remove the guest solvents. All N-Ts aryl aldimines were prepared according to reported procedures. ${ }^{[3]}$

To a flame-dried Schlenk Pressure Tube was added Spiro-1 (5 mg, $0.0014 \mathrm{mmol}$ ), N-sulfonyl aldimines $(0.1 \mathrm{mmol})$ and $0.5 \mathrm{~mL}$ anhydrous 1,2-dichloroethane. The mixture was stirred at $40{ }^{\circ} \mathrm{C}$ under $\mathrm{N}_{2}$ atmosphere for $30 \mathrm{~min}$. indole $(0.3 \mathrm{mmol})$ in $0.5 \mathrm{~mL}$ anhydrous 1,2-dichloroethane was then added via syringe. The mixture was stirred at $40{ }^{\circ} \mathrm{C}$ until the reaction completed. Chromatography on silica gel (1:3, EA-PE, v/v) afforded the desired products.

\subsection{Asymmetric three-component tandem Friedel-Crafts reaction of Indole with Aldehydes and $p$-Toluenesulfonamide}

The catalysts Spiro-1 and Spiro-2 were exchanged with acetone thoroughly and then activated at $220{ }^{\circ} \mathrm{C}$ to generate the Lewis acidic $\mathrm{Zr}(\mathrm{IV})$ sites .

To a flame-dried Schlenk Pressure Tube was added Spiro-1 (5 mg, $0.0014 \mathrm{mmol}$ ), $p$-Toluenesulfonamide $(0.11 \mathrm{mmol})$, aldehydes $(0.1 \mathrm{mmol})$, indole $(0.3 \mathrm{mmol})$ and $1 \mathrm{~mL}$ anhydrous 1,2-dichloroethane. The mixture was stirred at $80{ }^{\circ} \mathrm{C}$ until the reaction completed. Chromatography on silica gel (1:3, EA-PE, v/v) afforded the desired products.

3.6 Asymmetric iso-Pictet-Spengler reaction of $o$-aminobenzylindole with trifluoromethyl ketones

The catalysts Spiro-1 and Spiro-2 were exchanged with acetone thoroughly and then activated at $60{ }^{\circ} \mathrm{C}$ to remove the guest solvents. $o$-aminobenzylindole was prepared according to reported procedures. ${ }^{[4]}$

To a flame-dried Schlenk Pressure Tube was added Spiro-1 (8 $\mathrm{mg}, 0.0022 \mathrm{mmol}$ ), $o$-aminobenzylindole $(0.1 \mathrm{mmol})$, anhydrous $\mathrm{MgSO}_{4}(100 \mathrm{mg})$ and $0.5 \mathrm{~mL}$ anhydrous $\mathrm{CHCl}_{3}$. The reaction mixture was stirred at $60{ }^{\circ} \mathrm{C}$ for $30 \mathrm{~min}$ and trifluoromethyl ketones $(0.15 \mathrm{mmol})$ in 0.5 $\mathrm{mL} \mathrm{CHCl}_{3}$ was added via syringe. The mixture was stirred at $60{ }^{\circ} \mathrm{C}$ until the reaction completed. Chromatography on silica gel (1:10, EA-PE, v/v) afforded the desired products.

\subsection{General procedure for recycling experiments}

3.7.1 Asymmetric condensation/amine addition cascade sequence of 2 -aminobenzamide with 4-bromobenzaldehyde

To a flame-dried Schlenk Pressure Tube was added Spiro-1 (40 mg, $0.0112 \mathrm{mmol}$ ), 2-aminobenzamide $(0.8 \mathrm{mmol})$, anhydrous $\mathrm{MgSO}_{4}(100 \mathrm{mg})$ and $2 \mathrm{~mL}$ anhydrous $\mathrm{CHCl}_{3}$, and the mixture was stirred at $60{ }^{\circ} \mathrm{C}$ for $30 \mathrm{~min}$. 4-bromobenzaldehyde $(1.2 \mathrm{mmol})$ in $2 \mathrm{~mL}$ anhydrous $\mathrm{CHCl}_{3}$ was added via syringe, and the reaction mixture was stirred at $60{ }^{\circ} \mathrm{C}$ until the reaction completed. The reaction mixture was centrifuged at $14,000 \mathrm{rpm}$ for $10 \mathrm{~min}$ to recover the catalyst. The supernatant was concentrated and purified by flash chromatography to afford the desired product. The recovered Spiro-1 was washed with fresh water, ethanol and acetone for three times, reactivated at $60{ }^{\circ} \mathrm{C}$ for $2 \mathrm{~h}$ and then used for the following runs directly. 
3.7.2 Asymmetric Tandem Friedel-Crafts reaction of indole with 4-bromobenzaldehyde and $p$-toluenesulfonamide

To a flame-dried Schlenk Pressure Tube was added Spiro-1 (40 mg, $0.0112 \mathrm{mmol}$ ), 4-bromobenzaldehyde $(0.8 \mathrm{mmol}), p$-toluenesulfonamide $(0.88 \mathrm{mmol})$, indole $(2.4 \mathrm{mmol})$ and 4 $\mathrm{mL}$ anhydrous DCE, the reaction mixture was stirred at $80{ }^{\circ} \mathrm{C}$ until the reaction completed. The reaction mixture was centrifuged at $14,000 \mathrm{rpm}$ for $10 \mathrm{~min}$ to recover the catalyst. The supernatant was concentrated and purified by flash chromatography to afford the desired product. The recovered Spiro-1 was washed with fresh ethanol and acetone for three times, reactivated at $220^{\circ} \mathrm{C}$ overnight and then used for the following runs directly.

3.7.3 Asymmetric iso-Pictet-Spengler reaction of $o$-aminobenzylindole with trifluoroacetophenone

To a flame-dried Schlenk Pressure Tube was added Spiro-1 (40 mg, $0.011 \mathrm{mmol}$ ), $o$-aminobenzylindole $(0.5 \mathrm{mmol})$ and $2 \mathrm{~mL}$ anhydrous $\mathrm{CHCl}_{3}$, and the mixture was stirred at $60{ }^{\circ} \mathrm{C}$ for $30 \mathrm{~min}$. Trifluoroacetophenone $(0.75 \mathrm{mmol})$ in $2 \mathrm{~mL}$ anhydrous $\mathrm{CHCl}_{3}$ was added via syringe, and the reaction mixture was stirred at $60{ }^{\circ} \mathrm{C}$ until the reaction completed. The reaction mixture was centrifuged at $14,000 \mathrm{rpm}$ for $10 \mathrm{~min}$ to recover the catalyst. The supernatant was concentrated and purified by flash chromatography to afford the desired product. The recovered Spiro-1 was washed with fresh water, ethanol and acetone for three times, reactivated at $60{ }^{\circ} \mathrm{C}$ for $2 \mathrm{~h}$ and then used for the following runs directly.

\section{Table S1. Crystal data and structure refinement}

\begin{tabular}{|c|c|c|}
\hline Identification Code & Spiro-1 & Spiro-2 \\
\hline Empirical formula & $\mathrm{C}_{135} \mathrm{H}_{78} \mathrm{O}_{60} \mathrm{P}_{3} \mathrm{Zr}_{9}$ & $\mathrm{C}_{183} \mathrm{H}_{102} \mathrm{O}_{60} \mathrm{P}_{3} \mathrm{Zr}_{9}$ \\
\hline Formula weight & 3573.86 & 4174.53 \\
\hline Temperature (K) & 173.0 & 173.0 \\
\hline Wavelength $(\AA)$ & 1.54178 & 0.71073 \\
\hline Crystal system & Hexagonal & Trigonal \\
\hline Space group & $P 6_{2} 22$ & $P 3_{2} 21$ \\
\hline Unit cell dimensions & $\begin{array}{l}a=38.1622(9) \AA \alpha=90^{\circ} \\
b=38.1622(9) \AA \beta=90^{\circ} \\
c=45.5859(9)(15) \AA \gamma=120^{\circ}\end{array}$ & $\begin{array}{l}a=45.122(6) \AA \alpha=90^{\circ} \\
b=45.122(6) \AA \beta=90^{\circ} \\
c=49.238(10) \AA \gamma=120^{\circ}\end{array}$ \\
\hline Volume $\left(\AA^{3}\right), Z$ & $57495(3), 6$ & $86818(30), 6$ \\
\hline $\begin{array}{l}\text { Density (calculated) } \\
\left(\mathrm{mg} / \mathrm{m}^{3}\right)\end{array}$ & 0.619 & 0.479 \\
\hline $\begin{array}{l}\text { Absorption } \\
\text { coefficient }\left(\mathrm{mm}^{-1}\right)\end{array}$ & 2.346 & 0.191 \\
\hline$F(000)$ & 10638 & 12498 \\
\hline $\begin{array}{l}\text { Reflections collected } \\
\text { / unique }\end{array}$ & $163520 / 15251$ & $105991 / 55181$ \\
\hline $\begin{array}{l}\text { Completeness to } \\
\text { theta }\end{array}$ & $44.727,99.7 \%$ & $25.242,100 \%$ \\
\hline$R_{i n t}$ & 0.1803 & 0.0266 \\
\hline
\end{tabular}




\begin{tabular}{|l|l|l|}
\hline Refinement method & Full-matrix least-squares on $\mathrm{F}^{\wedge} 2$ & Full-matrix least-squares on $\mathrm{F}^{\wedge} 2$ \\
\hline $\begin{array}{l}\text { Data / restraints / } \\
\text { parameters }\end{array}$ & $15251 / 2025 / 802$ & $55181 / 5333 / 1901$ \\
\hline $\begin{array}{l}\text { Goodness-of-fit on } \\
\mathrm{F}^{2}\end{array}$ & 1.026 & 0.964 \\
\hline $\begin{array}{l}\text { Final } R \text { indices } \\
{[\mathrm{I}>2 \text { sigma(I) }]}\end{array}$ & $R_{1}=0.0755, w R_{2}=0.1676$ & $R_{1}=0.0872, w R_{2}=0.2634$ \\
\hline$R$ indices (all data) & $R_{1}=0.1096, w R_{2}=0.1797$ & $R_{1}=0.1213, w R_{2}=0.2879$ \\
\hline $\begin{array}{l}\text { Absolute structure } \\
\text { parameter }\end{array}$ & $0.060(10)$ & $-0.06(4)$ \\
\hline $\begin{array}{l}\text { Largest diff. peak } \\
\left.\text { and hole (e. } \AA^{-3}\right)\end{array}$ & 0.791 and -0.923 & 0.932 and -1.023 \\
\hline
\end{tabular}

5. Table S2. Selected bond lengths $[\AA]$ and angles $\left[{ }^{\circ}\right]$ for Spiro-1

\begin{tabular}{|l|l|l|l|}
\hline $\mathrm{Zr}(1)-\mathrm{O}(18) \# 9$ & $2.097(12)$ & $\mathrm{Zr}(1)-\mathrm{O}(19) \# 4$ & $2.113(12)$ \\
\hline $\mathrm{Zr}(2)-\mathrm{O}(16) \# 4$ & $2.273(13)$ & $\mathrm{Zr}(2)-\mathrm{O}(16) \# 9$ & $2.273(13)$ \\
\hline $\mathrm{Zr}(2)-\mathrm{O}(18) \# 4$ & $2.213(11)$ & $\mathrm{Zr}(2)-\mathrm{O}(18) \# 9$ & $2.213(11)$ \\
\hline $\mathrm{Zr}(2)-\mathrm{O}(19) \# 9$ & $2.189(11)$ & $\mathrm{Zr}(2)-\mathrm{O}(19) \# 4$ & $2.188(11)$ \\
\hline $\mathrm{Zr}(2)-\mathrm{O}(23) \# 11$ & $2.208(11)$ & $\mathrm{Zr}(3)-\mathrm{O}(1) \# 2$ & $2.118(12)$ \\
\hline $\mathrm{Zr}(3)-\mathrm{O}(13) \# 6$ & $2.288(13)$ & $\mathrm{Zr}(4)-\mathrm{O}(1) \# 12$ & $2.231(11)$ \\
\hline $\mathrm{Zr}(4)-\mathrm{O}(2) \# 12$ & $2.190(11)$ & $\mathrm{Zr}(4)-\mathrm{O}(5) \# 4$ & $2.187(12)$ \\
\hline $\mathrm{Zr}(4)-\mathrm{O}(7) \# 4$ & $2.105(11)$ & $\mathrm{Zr}(4)-\mathrm{O}(12) \# 13$ & $2.171(13)$ \\
\hline $\mathrm{Zr}(4)-\mathrm{O}(15) \# 8$ & $2.168(12)$ & $\mathrm{Zr}(5)-\mathrm{O}(2) \# 4$ & $2.087(14)$ \\
\hline $\mathrm{Zr}(5)-\mathrm{O}(5) \# 4$ & $2.088(13)$ & $\mathrm{Zr}(5)-\mathrm{O}(14) \# 14$ & $2.256(13)$ \\
\hline $\mathrm{Zr}(5)-\mathrm{O}(28) \# 10$ & $2.157(13)$ & & \\
\hline & & & 73.8 \\
\hline $\mathrm{O}(17)-\mathrm{Zr}(1)-\mathrm{O}(22)$ & $128.4(4)$ & $\mathrm{O}(3)-\mathrm{Zr}(3)-\mathrm{O}(6)$ & $73.8(4)$ \\
\hline $\mathrm{O}(18) \# 9-\mathrm{Zr}(1)-\mathrm{O}(17)$ & $138.7(4)$ & $\mathrm{O}(3)-\mathrm{Zr}(3)-\mathrm{O}(13) \# 6$ & $70.4(4)$ \\
\hline $\mathrm{O}(18)-\mathrm{Zr}(1)-\mathrm{O}(17)$ & $77.1(4)$ & $\mathrm{O}(4)-\mathrm{Zr}(3)-\mathrm{O}(1)$ & $88.9(4)$ \\
\hline $\mathrm{O}(18) \# 9-\mathrm{Zr}(1)-\mathrm{O}(18)$ & $65.5(5)$ & $\mathrm{O}(4)-\mathrm{Zr}(3)-\mathrm{O}(1) \# 2$ & $88.0(4)$ \\
\hline $\mathrm{O}(18) \# 9-\mathrm{Zr}(1)-\mathrm{O}(19) \# 4$ & $72.8(4)$ & $\mathrm{O}(4)-\mathrm{Zr}(3)-\mathrm{O}(3)$ & $91.7(4)$ \\
\hline $\mathrm{O}(18) \# 9-\mathrm{Zr}(1)-\mathrm{O}(19)$ & $107.9(4)$ & $\mathrm{O}(4)-\mathrm{Zr}(3)-\mathrm{O}(5)$ & $146.8(4)$ \\
\hline $\mathrm{O}(18)-\mathrm{Zr}(1)-\mathrm{O}(19)$ & $72.9(4)$ & $\mathrm{O}(4)-\mathrm{Zr}(3)-\mathrm{O}(6)$ & $72.0(4)$ \\
\hline $\mathrm{O}(18) \# 9-\mathrm{Zr}(1)-\mathrm{O}(20)$ & $88.8(4)$ & $\mathrm{O}(4)-\mathrm{Zr}(3)-\mathrm{O}(13) \# 6$ & $72.6(4)$ \\
\hline $\mathrm{O}(18)-\mathrm{Zr}(1)-\mathrm{O}(20)$ & $89.3(4)$ & $\mathrm{O}(5)-\mathrm{Zr}(3)-\mathrm{O}(1) \# 2$ & $74.0(4)$ \\
\hline $\mathrm{O}(18)-\mathrm{Zr}(1)-\mathrm{O}(21)$ & $147.1(5)$ & $\mathrm{O}(5)-\mathrm{Zr}(3)-\mathrm{O}(1)$ & $107.7(4)$ \\
\hline $\mathrm{O}(18) \# 9-\mathrm{Zr}(1)-\mathrm{O}(21)$ & $147.2(5)$ & $\mathrm{O}(5)-\mathrm{Zr}(3)-\mathrm{O}(3)$ & $90.6(4)$ \\
\hline $\mathrm{O}(18)-\mathrm{Zr}(1)-\mathrm{O}(22)$ & $139.4(4)$ & $\mathrm{O}(5)-\mathrm{Zr}(3)-\mathrm{O}(6)$ & $76.9(4)$ \\
\hline $\mathrm{O}(18) \# 9-\mathrm{Zr}(1)-\mathrm{O}(22)$ & $77.6(4)$ & $\mathrm{O}(5)-\mathrm{Zr}(3)-\mathrm{O}(13) \# 6$ & $138.4(5)$ \\
\hline $\mathrm{O}(18) \# 9-\mathrm{Zr}(1)-\mathrm{Zr}(1) \# 9$ & $32.9(3)$ & $\mathrm{O}(6)-\mathrm{Zr}(3)-\mathrm{O}(13) \# 6$ & $127.9(5)$ \\
\hline $\mathrm{O}(19)-\mathrm{Zr}(1)-\mathrm{O}(17)$ & $75.4(5)$ & $\mathrm{O}(2) \# 12-\mathrm{Zr}(4)-\mathrm{O}(1) \# 12$ & $70.1(5)$ \\
\hline $\mathrm{O}(19) \# 4-\mathrm{Zr}(1)-\mathrm{O}(17)$ & $138.5(5)$ & $\mathrm{O}(2) \# 12-\mathrm{Zr}(4)-\mathrm{O}(26)$ & $141.1(4)$ \\
\hline $\mathrm{O}(19) \# 4-\mathrm{Zr}(1)-\mathrm{O}(18)$ & $107.9(5)$ & $\mathrm{O}(5) \# 4-\mathrm{Zr}(4)-\mathrm{O}(1) \# 12$ & $70.3(4)$ \\
\hline & & & \\
\hline
\end{tabular}




\begin{tabular}{|l|l|l|l|}
\hline $\mathrm{O}(19) \# 4-\mathrm{Zr}(1)-\mathrm{O}(19)$ & $67.6(5)$ & $\mathrm{O}(5) \# 4-\mathrm{Zr}(4)-\mathrm{O}(2) \# 12$ & $106.5(4)$ \\
\hline $\mathrm{O}(19) \# 4-\mathrm{Zr}(1)-\mathrm{O}(20)$ & $145.7(5)$ & $\mathrm{O}(5) \# 4-\mathrm{Zr}(4)-\mathrm{O}(26)$ & $80.9(4)$ \\
\hline $\mathrm{O}(19)-\mathrm{Zr}(1)-\mathrm{O}(20)$ & $146.7(5)$ & $\mathrm{O}(7) \# 4-\mathrm{Zr}(4)-\mathrm{O}(1) \# 12$ & $79.9(4)$ \\
\hline
\end{tabular}

Symmetry transformations used to generate equivalent atoms:

$\# 1 \mathrm{x}, \mathrm{x}-\mathrm{y},-\mathrm{z}+1 / 3 \quad \# 2-\mathrm{x},-\mathrm{y}+1, \mathrm{z} \quad \# 3 \mathrm{x}-\mathrm{y},-\mathrm{y}+1,-\mathrm{z} \quad \# 4-\mathrm{x}+\mathrm{y}, \mathrm{y},-\mathrm{z} \quad \# 5 \mathrm{x}-\mathrm{y}, \mathrm{x}, \mathrm{z}+1 / 3 \quad$ \#6 $\mathrm{x}, \mathrm{x}-\mathrm{y}+1,-\mathrm{z}+1 / 3 \quad \# 7 \mathrm{x}-1, \mathrm{x}-\mathrm{y},-\mathrm{z}+1 / 3 \quad \# 8-\mathrm{x},-\mathrm{x}+\mathrm{y},-\mathrm{z}+1 / 3 \quad \# 9-\mathrm{x},-\mathrm{y}, \mathrm{z} \quad \# 10-\mathrm{x}+1,-\mathrm{y}+1, \mathrm{z}$ $\# 11 \mathrm{x}-\mathrm{y},-\mathrm{y},-\mathrm{z} \quad \# 12 \mathrm{x}-\mathrm{y}+1,-\mathrm{y}+1,-\mathrm{z} \quad \# 13 \mathrm{y},-\mathrm{x}+\mathrm{y}, \mathrm{z}-1 / 3 \quad \# 14 \mathrm{x}+1, \mathrm{x}-\mathrm{y}+1,-\mathrm{z}+1 / 3$

6. Table S3. Selected bond lengths $[\AA ̊ \cap]$ and angles $\left[{ }^{\circ}\right]$ for Spiro-2

\begin{tabular}{|l|l|l|l|}
\hline $\mathrm{Zr}(1)-\mathrm{O}(17) \# 1$ & $2.064(4)$ & $\mathrm{Zr}(7)-\mathrm{O}(44)$ & $2.033(3)$ \\
\hline $\mathrm{Zr}(1)-\mathrm{O}(17)$ & $2.064(4)$ & $\mathrm{Zr}(7)-\mathrm{O}(52)$ & $2.270(5)$ \\
\hline $\mathrm{Zr}(1)-\mathrm{O}(13)$ & $2.159(3)$ & $\mathrm{Zr}(7)-\mathrm{O}(46)$ & $2.061(4)$ \\
\hline $\mathrm{Zr}(1)-\mathrm{O}(13) \# 1$ & $2.159(3)$ & $\mathrm{Zr}(7)-\mathrm{O}(51)$ & $2.083(3)$ \\
\hline $\mathrm{Zr}(1)-\mathrm{O}(15) \# 1$ & $2.356(3)$ & $\mathrm{Zr}(7)-\mathrm{O}(31) \# 6$ & $2.368(6)$ \\
\hline $\mathrm{Zr}(1)-\mathrm{O}(15)$ & $2.356(3)$ & $\mathrm{Zr}(7)-\mathrm{O}(47)$ & $2.312(4)$ \\
\hline $\mathrm{Zr}(1)-\mathrm{O}(11)$ & $2.223(4)$ & $\mathrm{Zr}(7)-\mathrm{O}(45)$ & $2.166(5)$ \\
\hline $\mathrm{Zr}(1)-\mathrm{O}(11) \# 1$ & $2.223(4)$ & $\mathrm{Zr}(8)-\mathrm{O}(41)$ & $2.072(3)$ \\
\hline $\mathrm{Zr}(2)-\mathrm{O}(19) \# 1$ & $2.262(3)$ & $\mathrm{Zr}(8)-\mathrm{O}(43)$ & $2.318(4)$ \\
\hline $\mathrm{Zr}(2)-\mathrm{O}(19)$ & $2.262(3)$ & $\mathrm{Zr}(8)-\mathrm{O}(44)$ & $2.074(3)$ \\
\hline $\mathrm{Zr}(2)-\mathrm{O}(21) \# 1$ & $2.002(3)$ & $\mathrm{Zr}(8)-\mathrm{O}(34) \# 8$ & $2.245(7)$ \\
\hline $\mathrm{Zr}(2)-\mathrm{O}(21)$ & $2.002(3)$ & $\mathrm{Zr}(8)-\mathrm{O}(35)$ & $2.273(5)$ \\
\hline $\mathrm{Zr}(2)-\mathrm{O}(24) \# 2$ & $2.139(4)$ & $\mathrm{Zr}(8)-\mathrm{O}(29) \# 5$ & $2.281(4)$ \\
\hline $\mathrm{Zr}(2)-\mathrm{O}(24) \# 3$ & $2.139(4)$ & $\mathrm{Zr}(8)-\mathrm{O}(36)$ & $2.203(5)$ \\
\hline $\mathrm{Zr}(2)-\mathrm{O}(9) \# 2$ & $2.185(4)$ & $\mathrm{Zr}(8)-\mathrm{O}(53)$ & $2.234(4)$ \\
\hline $\mathrm{Zr}(2)-\mathrm{O}(9) \# 3$ & $2.185(4)$ & $\mathrm{Zr}(9)-\mathrm{O}(50)$ & $2.159(4)$ \\
\hline $\mathrm{Zr}(3)-\mathrm{O}(17)$ & $2.117(4)$ & $\mathrm{Zr}(9)-\mathrm{O}(7)$ & $2.230(6)$ \\
\hline $\mathrm{Zr}(3)-\mathrm{O}(19)$ & $2.155(3)$ & $\mathrm{Zr}(9)-\mathrm{O}(49)$ & $2.188(4)$ \\
\hline $\mathrm{Zr}(3)-\mathrm{O}(21) \# 1$ & $2.138(3)$ & $\mathrm{Zr}(9)-\mathrm{O}(54)$ & $2.146(4)$ \\
\hline $\mathrm{Zr}(3)-\mathrm{O}(10) \# 2$ & $2.178(4)$ & $\mathrm{Zr}(9)-\mathrm{O}(52)$ & $2.100(5)$ \\
\hline $\mathrm{Zr}(3)-\mathrm{O}(16)$ & $2.253(4)$ & $\mathrm{Zr}(9)-\mathrm{O}(51)$ & $2.055(3)$ \\
\hline $\mathrm{Zr}(3)-\mathrm{O}(14)$ & $2.296(4)$ & $\mathrm{Zr}(9)-\mathrm{O}(56) \# 4$ & $2.221(5)$ \\
\hline $\mathrm{Zr}(3)-\mathrm{O}(15)$ & $2.240(3)$ & $\mathrm{Zr}(9)-\mathrm{O}(42)$ & $2.071(4)$ \\
\hline $\mathrm{Zr}(3)-\mathrm{O}(18)$ & $2.124(3)$ & $\mathrm{Zr}(10)-\mathrm{O}(41)$ & $2.067(3)$ \\
\hline $\mathrm{Zr}(4)-\mathrm{O}(17)$ & $2.031(4)$ & $\mathrm{Zr}(10)-\mathrm{O}(39)$ & $2.239(5)$ \\
\hline $\mathrm{Zr}(4)-\mathrm{O}(19)$ & $2.266(3)$ & $\mathrm{Zr}(10)-\mathrm{O}(37)$ & $2.193(6)$ \\
\hline $\mathrm{Zr}(4)-\mathrm{O}(21)$ & $2.143(3)$ & $\mathrm{Zr}(10)-\mathrm{O}(54)$ & $2.331(4)$ \\
\hline $\mathrm{Zr}(4)-\mathrm{O}(15) \# 1$ & $2.128(3)$ & $\mathrm{Zr}(10)-\mathrm{O}(2) \# 7$ & $2.252(5)$ \\
\hline $\mathrm{Zr}(4)-\mathrm{O}(12) \# 1$ & $2.223(4)$ & $\mathrm{Zr}(10)-\mathrm{O}(40)$ & $2.210(4)$ \\
\hline $\mathrm{Zr}(4)-\mathrm{O}(22)$ & $2.246(3)$ & $\mathrm{Zr}(10)-\mathrm{O}(53)$ & $2.225(4)$ \\
\hline $\mathrm{Zr}(4)-\mathrm{O}(20)$ & $2.140(3)$ & $\mathrm{Zr}(10)-\mathrm{O}(42)$ & $2.100(4)$ \\
\hline $\mathrm{Zr}(4)-\mathrm{O}(23) \# 2$ & $2.175(4)$ & $2.236(4)$ \\
\hline $\mathrm{Zr}(5)-\mathrm{O}(55) \# 4$ & $2.161(4)$ & $2.076(4)$ \\
\hline & & & \\
\hline
\end{tabular}




\begin{tabular}{|c|c|c|c|}
\hline $\mathrm{Zr}(5)-\mathrm{O}(44)$ & $2.045(3)$ & $\mathrm{Zr}(6)-\mathrm{O}(33) \# 8$ & $2.174(4)$ \\
\hline $\mathrm{Zr}(5)-\mathrm{O}(52)$ & $2.108(5)$ & $\mathrm{Zr}(6)-\mathrm{O}(41)$ & $2.031(3)$ \\
\hline $\mathrm{Zr}(5)-\mathrm{O}(30) \# 5$ & $2.153(4)$ & $\mathrm{Zr}(6)-\mathrm{O}(43)$ & $2.296(4)$ \\
\hline $\mathrm{Zr}(5)-\mathrm{O}(32) \# 6$ & $2.098(4)$ & $\mathrm{Zr}(6)-\mathrm{O}(54)$ & $2.357(4)$ \\
\hline $\mathrm{Zr}(5)-\mathrm{O}(1) \# 7$ & $2.177(3)$ & $\mathrm{Zr}(6)-\mathrm{O}(8)$ & $2.213(4)$ \\
\hline $\mathrm{Zr}(6)-\mathrm{O}(38)$ & $2.231(4)$ & $\mathrm{Zr}(6)-\mathrm{O}(48)$ & $2.181(3)$ \\
\hline $\mathrm{Zr}(7)-\mathrm{O}(43)$ & $2.188(4)$ & $\mathrm{Zr}(6)-\mathrm{O}(51)$ & $1.992(3)$ \\
\hline $\mathrm{O}(17) \# 1-\mathrm{Zr}(1)-\mathrm{O}(17)$ & $97.2(2)$ & $\mathrm{O}(33) \# 8-\mathrm{Zr}(6)-\mathrm{O}(8)$ & $118.30(13)$ \\
\hline $\mathrm{O}(17) \# 1-\mathrm{Zr}(1)-\mathrm{O}(13) \# 1$ & $84.36(15)$ & $\mathrm{O}(33) \# 8-\mathrm{Zr}(6)-\mathrm{O}(48)$ & $74.47(12)$ \\
\hline $\mathrm{O}(17)-\mathrm{Zr}(1)-\mathrm{O}(13) \# 1$ & $143.02(14)$ & $\mathrm{O}(33) \# 8-\mathrm{Zr}(6)-\mathrm{O}(38)$ & $70.16(14)$ \\
\hline $\mathrm{O}(17)-\mathrm{Zr}(1)-\mathrm{O}(13)$ & $84.36(15)$ & $\mathrm{O}(41)-\mathrm{Zr}(6)-\mathrm{O}(33) \# 8$ & $85.43(15)$ \\
\hline $\mathrm{O}(17) \# 1-\mathrm{Zr}(1)-\mathrm{O}(13)$ & $143.02(14)$ & $\mathrm{O}(41)-\mathrm{Zr}(6)-\mathrm{O}(43)$ & $72.50(14)$ \\
\hline $\mathrm{O}(17) \# 1-\mathrm{Zr}(1)-\mathrm{O}(15)$ & $68.13(14)$ & $\mathrm{O}(41)-\mathrm{Zr}(6)-\mathrm{O}(54)$ & $73.35(14)$ \\
\hline $\mathrm{O}(17) \# 1-\mathrm{Zr}(1)-\mathrm{O}(15) \# 1$ & \begin{tabular}{|l|}
$73.48(14)$ \\
\end{tabular} & $\mathrm{O}(41)-\mathrm{Zr}(6)-\mathrm{O}(8)$ & $140.62(13)$ \\
\hline $\mathrm{O}(17)-\mathrm{Zr}(1)-\mathrm{O}(15)$ & $73.48(14)$ & $\mathrm{O}(41)-\mathrm{Zr}(6)-\mathrm{O}(48)$ & $142.59(13)$ \\
\hline $\mathrm{O}(17)-\mathrm{Zr}(1)-\mathrm{O}(15) \# 1$ & 68.13(14) & $\mathrm{O}(41)-\mathrm{Zr}(6)-\mathrm{O}(38)$ & $85.21(16)$ \\
\hline $\mathrm{O}(17)-\mathrm{Zr}(1)-\mathrm{O}(11)$ & $146.55(15)$ & $\mathrm{O}(43)-\mathrm{Zr}(6)-\mathrm{O}(54)$ & $124.59(14)$ \\
\hline $\mathrm{O}(17)-\mathrm{Zr}(1)-\mathrm{O}(11) \# 1$ & $83.34(16)$ & $\mathrm{O}(8)-\mathrm{Zr}(6)-\mathrm{O}(43)$ & $140.53(13)$ \\
\hline $\mathrm{O}(17) \# 1-\mathrm{Zr}(1)-\mathrm{O}(11) \# 1$ & $146.55(15)$ & $\mathrm{O}(8)-\mathrm{Zr}(6)-\mathrm{O}(54)$ & $68.98(13)$ \\
\hline $\mathrm{O}(17) \# 1-\mathrm{Zr}(1)-\mathrm{O}(11)$ & $83.34(16)$ & $\mathrm{O}(8)-\mathrm{Zr}(6)-\mathrm{O}(38)$ & $75.59(14)$ \\
\hline $\mathrm{O}(13) \# 1-\mathrm{Zr}(1)-\mathrm{O}(13)$ & $116.03(18)$ & $\mathrm{O}(48)-\mathrm{Zr}(6)-\mathrm{O}(43)$ & $72.07(13)$ \\
\hline $\mathrm{O}(13) \# 1-\mathrm{Zr}(1)-\mathrm{O}(15) \# 1$ & $77.13(12)$ & $\mathrm{O}(48)-\mathrm{Zr}(6)-\mathrm{O}(54)$ & $139.05(13)$ \\
\hline $\mathrm{O}(13)-\mathrm{Zr}(1)-\mathrm{O}(15)$ & $77.13(12)$ & $\mathrm{O}(48)-\mathrm{Zr}(6)-\mathrm{O}(8)$ & $76.60(12)$ \\
\hline $\mathrm{O}(13)-\mathrm{Zr}(1)-\mathrm{O}(15) \# 1$ & $138.50(12)$ & $\mathrm{O}(48)-\mathrm{Zr}(6)-\mathrm{O}(38)$ & $115.67(14)$ \\
\hline $\mathrm{O}(13) \# 1-\mathrm{Zr}(1)-\mathrm{O}(15)$ & $138.49(12)$ & $\mathrm{O}(51)-\mathrm{Zr}(6)-\mathrm{O}(33) \# 8$ & $144.98(13)$ \\
\hline $\mathrm{O}(13)-\mathrm{Zr}(1)-\mathrm{O}(11)$ & $76.09(14)$ & $\mathrm{O}(51)-\mathrm{Zr}(6)-\mathrm{O}(41)$ & $90.72(15)$ \\
\hline $\mathrm{O}(13)-\mathrm{Zr}(1)-\mathrm{O}(11) \# 1$ & $70.43(14)$ & $\mathrm{O}(51)-\mathrm{Zr}(6)-\mathrm{O}(43)$ & $70.18(13)$ \\
\hline $\mathrm{O}(13) \# 1-\mathrm{Zr}(1)-\mathrm{O}(11)$ & $70.42(14)$ & $\mathrm{O}(51)-\mathrm{Zr}(6)-\mathrm{O}(54)$ & $68.01(13)$ \\
\hline $\mathrm{O}(13) \# 1-\mathrm{Zr}(1)-\mathrm{O}(11) \# 1$ & $76.09(14)$ & $\mathrm{O}(51)-\mathrm{Zr}(6)-\mathrm{O}(8)$ & $85.73(13)$ \\
\hline $\mathrm{O}(15)-\mathrm{Zr}(1)-\mathrm{O}(15) \# 1$ & $120.44(16)$ & $\mathrm{O}(51)-\mathrm{Zr}(6)-\mathrm{O}(48)$ & $88.38(12)$ \\
\hline $\mathrm{O}(11)-\mathrm{Zr}(1)-\mathrm{O}(15) \# 1$ & $141.64(12)$ & $\mathrm{O}(51)-\mathrm{Zr}(6)-\mathrm{O}(38)$ & $144.24(14)$ \\
\hline $\mathrm{O}(11)-\mathrm{Zr}(1)-\mathrm{O}(15)$ & $75.84(13)$ & $\mathrm{O}(38)-\mathrm{Zr}(6)-\mathrm{O}(43)$ & $140.25(14)$ \\
\hline $\mathrm{O}(11) \# 1-\mathrm{Zr}(1)-\mathrm{O}(15)$ & $141.64(12)$ & $\mathrm{O}(38)-\mathrm{Zr}(6)-\mathrm{O}(54)$ & $76.83(15)$ \\
\hline $\mathrm{O}(11) \# 1-\mathrm{Zr}(1)-\mathrm{O}(15) \# 1$ & $75.84(13)$ & $\mathrm{O}(43)-\mathrm{Zr}(7)-\mathrm{O}(52)$ & $116.83(16)$ \\
\hline $\mathrm{O}(11)-\mathrm{Zr}(1)-\mathrm{O}(11) \# 1$ & $114.2(2)$ & $\mathrm{O}(43)-\mathrm{Zr}(7)-\mathrm{O}(31) \# 6$ & $139.18(16)$ \\
\hline $\mathrm{O}(19)-\mathrm{Zr}(2)-\mathrm{O}(19) \# 1$ & $119.43(18)$ & $\mathrm{O}(43)-\mathrm{Zr}(7)-\mathrm{O}(47)$ & $74.32(14)$ \\
\hline $\mathrm{O}(21)-\mathrm{Zr}(2)-\mathrm{O}(19) \# 1$ & $70.21(11)$ & $\mathrm{O}(44)-\mathrm{Zr}(7)-\operatorname{Zr}(5)$ & $30.98(9)$ \\
\hline $\mathrm{O}(21) \# 1-\mathrm{Zr}(2)-\mathrm{O}(19)$ & $70.21(11)$ & $\mathrm{O}(44)-\mathrm{Zr}(7)-\mathrm{Zr}(6)$ & $78.48(11)$ \\
\hline $\mathrm{O}(21) \# 1-\mathrm{Zr}(2)-\mathrm{O}(19) \# 1$ & $72.63(11)$ & $\mathrm{O}(44)-\mathrm{Zr}(7)-\mathrm{Zr}(8)$ & $30.22(9)$ \\
\hline $\mathrm{O}(21)-\mathrm{Zr}(2)-\mathrm{O}(19)$ & $72.63(11)$ & $\mathrm{O}(44)-\mathrm{Zr}(7)-\mathrm{Zr}(9)$ & $79.32(11)$ \\
\hline $\mathrm{O}(21)-\mathrm{Zr}(2)-\mathrm{O}(21) \# 1$ & $101.66(16)$ & $\mathrm{O}(44)-\mathrm{Zr}(7)-\mathrm{O}(43)$ & $69.04(13)$ \\
\hline $\mathrm{O}(21) \# 1-\mathrm{Zr}(2)-\mathrm{O}(24) \# 3$ & $83.71(12)$ & $\mathrm{O}(44)-\mathrm{Zr}(7)-\mathrm{O}(52)$ & $65.29(15)$ \\
\hline
\end{tabular}




\begin{tabular}{|l|l|l|l|}
\hline $\mathrm{O}(21)-\mathrm{Zr}(2)-\mathrm{O}(24) \# 3$ & $141.19(12)$ & $\mathrm{O}(44)-\mathrm{Zr}(7)-\mathrm{O}(46)$ & $146.33(15)$ \\
\hline $\mathrm{O}(21)-\mathrm{Zr}(2)-\mathrm{O}(24) \# 2$ & $83.71(12)$ & $\mathrm{O}(44)-\mathrm{Zr}(7)-\mathrm{O}(51)$ & $85.62(14)$ \\
\hline $\mathrm{O}(21) \# 1-\mathrm{Zr}(2)-\mathrm{O}(24) \# 2$ & $141.19(12)$ & $\mathrm{O}(44)-\mathrm{Zr}(7)-\mathrm{O}(31) \# 6$ & $81.92(16)$ \\
\hline $\mathrm{O}(21)-\mathrm{Zr}(2)-\mathrm{O}(9) \# 3$ & $81.30(13)$ & $\mathrm{O}(44)-\mathrm{Zr}(7)-\mathrm{O}(47)$ & $143.34(14)$ \\
\hline $\mathrm{O}(21) \# 1-\mathrm{Zr}(2)-\mathrm{O}(9) \# 3$ & $143.14(12)$ & $\mathrm{O}(44)-\mathrm{Zr}(7)-\mathrm{O}(45)$ & $99.94(16)$ \\
\hline
\end{tabular}

Symmetry transformations used to generate equivalent atoms:

$\# 1 \mathrm{y}, \mathrm{x},-\mathrm{z} \quad \# 2-\mathrm{x}+\mathrm{y}+1,-\mathrm{x}+2, \mathrm{z}+1 / 3 \quad \# 3-\mathrm{x}+2,-\mathrm{x}+\mathrm{y}+1,-\mathrm{z}-1 / 3 \quad \# 4-\mathrm{y}+1, \mathrm{x}-\mathrm{y}+1, \mathrm{z}-1 / 3 \quad$ \#5

$-x+y,-x+2, z+1 / 3 \quad \# 6 y-1, x,-z \quad \# 7-x+1,-x+y+1,-z-1 / 3 \quad \# 8 x-y+1,-y+2,-z+1 / 3 \quad \# 9$

$-\mathrm{x}+\mathrm{y},-\mathrm{x}+1, \mathrm{z}+1 / 3 \quad \# 10-\mathrm{y}+2, \mathrm{x}-\mathrm{y}+1, \mathrm{z}-1 / 3 \quad \# 11-\mathrm{y}+2, \mathrm{x}-\mathrm{y}+2, \mathrm{z}-1 / 3 \quad \# 12 \mathrm{y}, \mathrm{x}+1,-\mathrm{z} \quad \# 13$

$-x+1,-x+y,-z-1 / 3$

\section{Figures S1-S3. Additional X-ray crystallographic structures}

7.1 Figure S1. Two different types of 8-connected $\mathrm{Zr}_{6}$ cluster: left: idealized $\mathrm{Zr}_{6}\left(\mu_{3}-\mathrm{O}\right)_{4}\left(\mu_{3}-\mathrm{OH}\right)_{4}$ cluster core with $D_{4 h}$ symmetry; right: idealized $\mathrm{Zr}_{6}$ cluster but distorted $\mu_{3}-\mathrm{O} / \mathrm{OH}$ polyhedron with $D_{2 d}$ symmetry

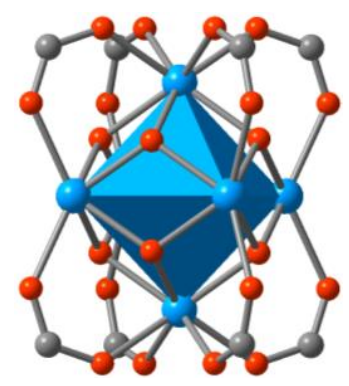

$D_{4 h}$ symmetry

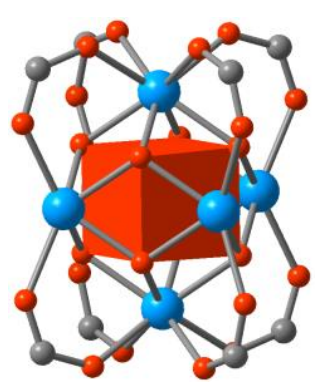

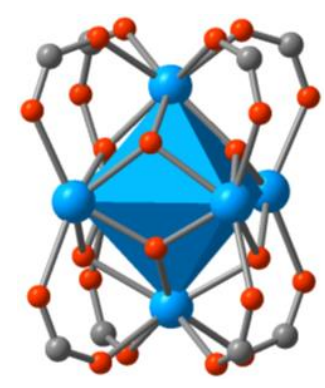

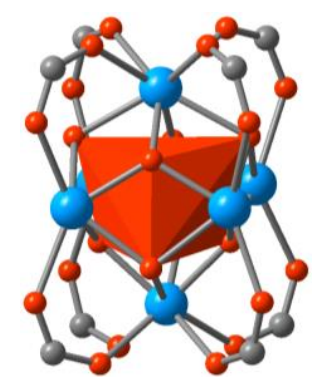

$D_{2 d}$ symmetry

7.2 Figure S2. Polyhedral representation showing the connectivity of cage (yellow cage share edge with neighboring four red cages and vertex with neighboring six blue cages)
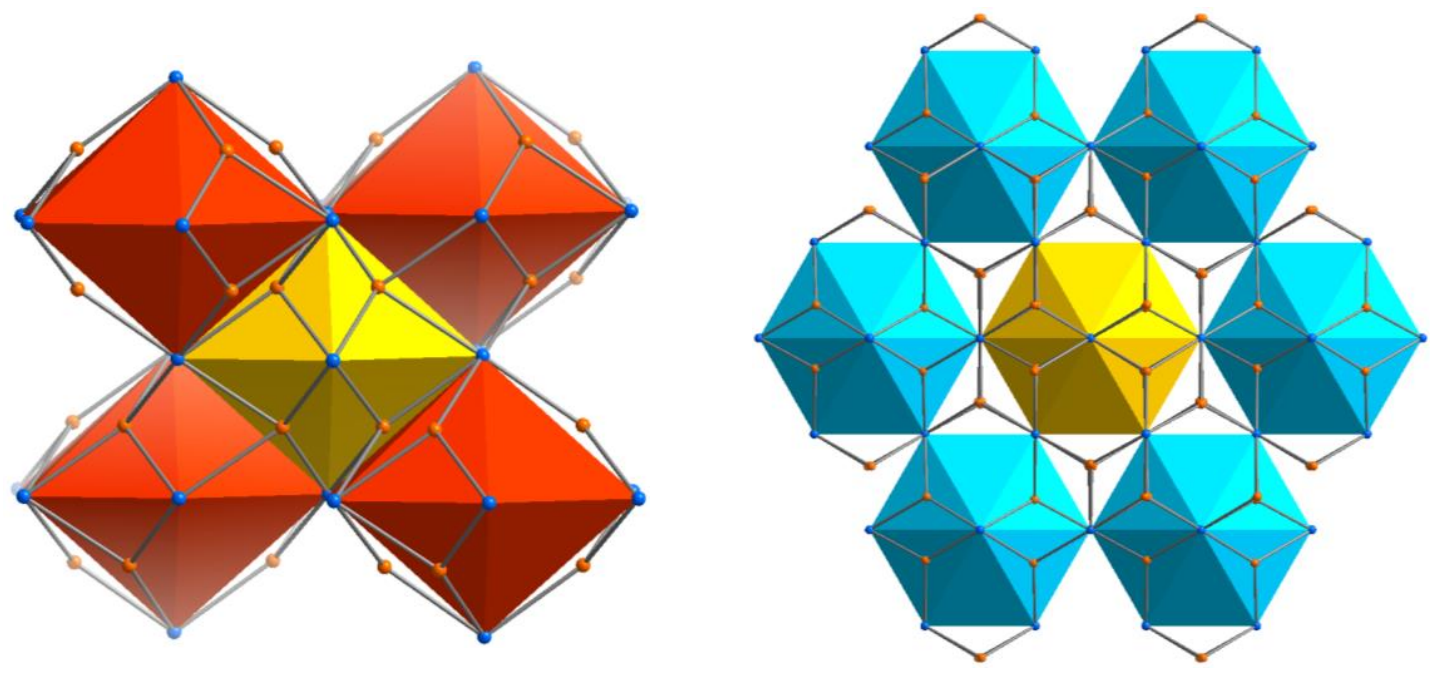
7.3 Figure S3. 3D structures of Spiro-2 viewed from $b$ axis and topology of Spiro-2
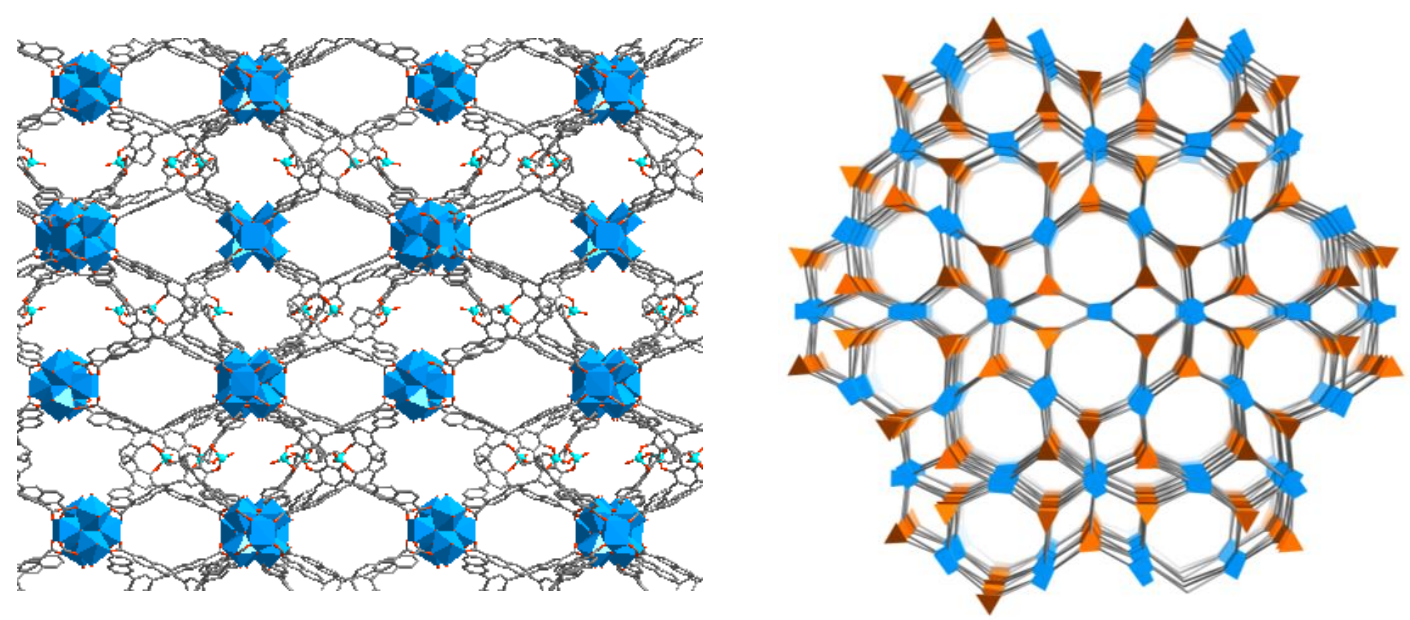

8. Figure S4. CD spectra.
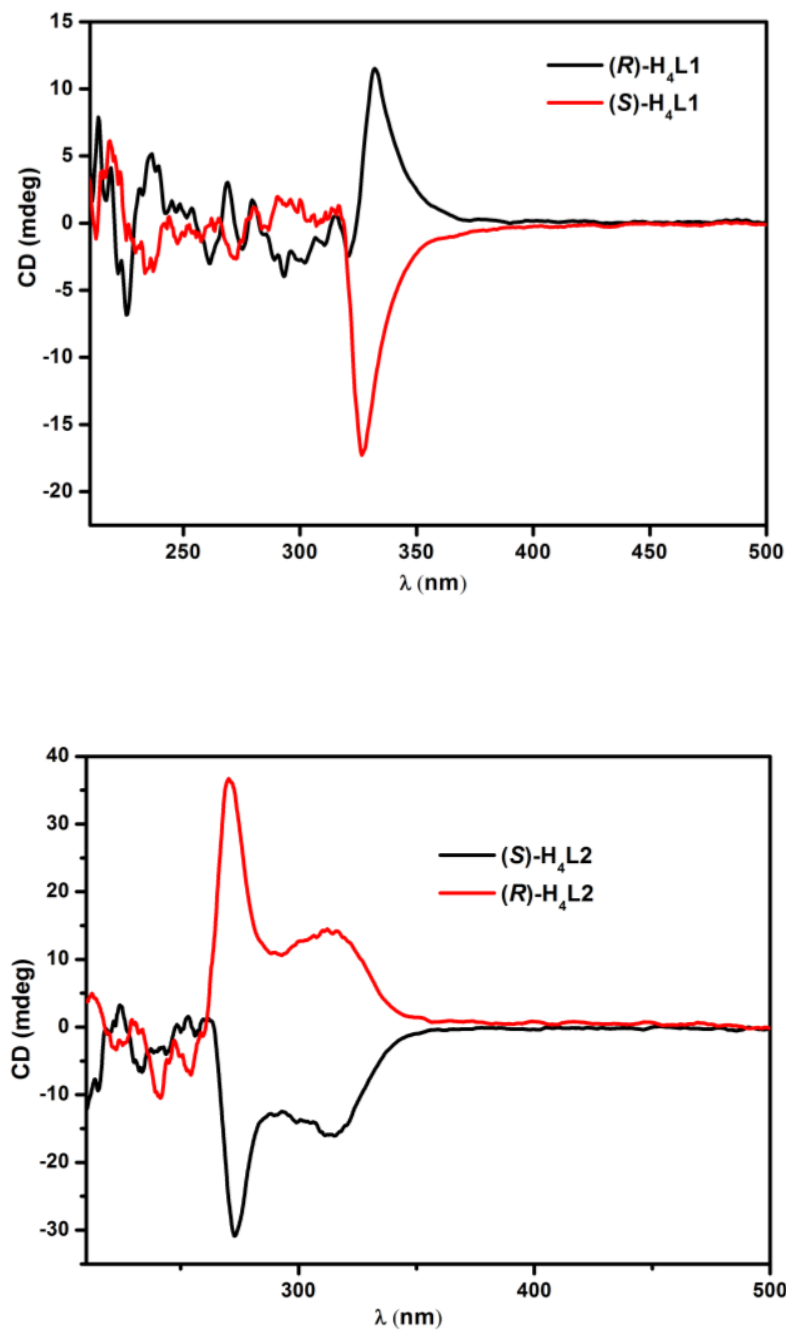

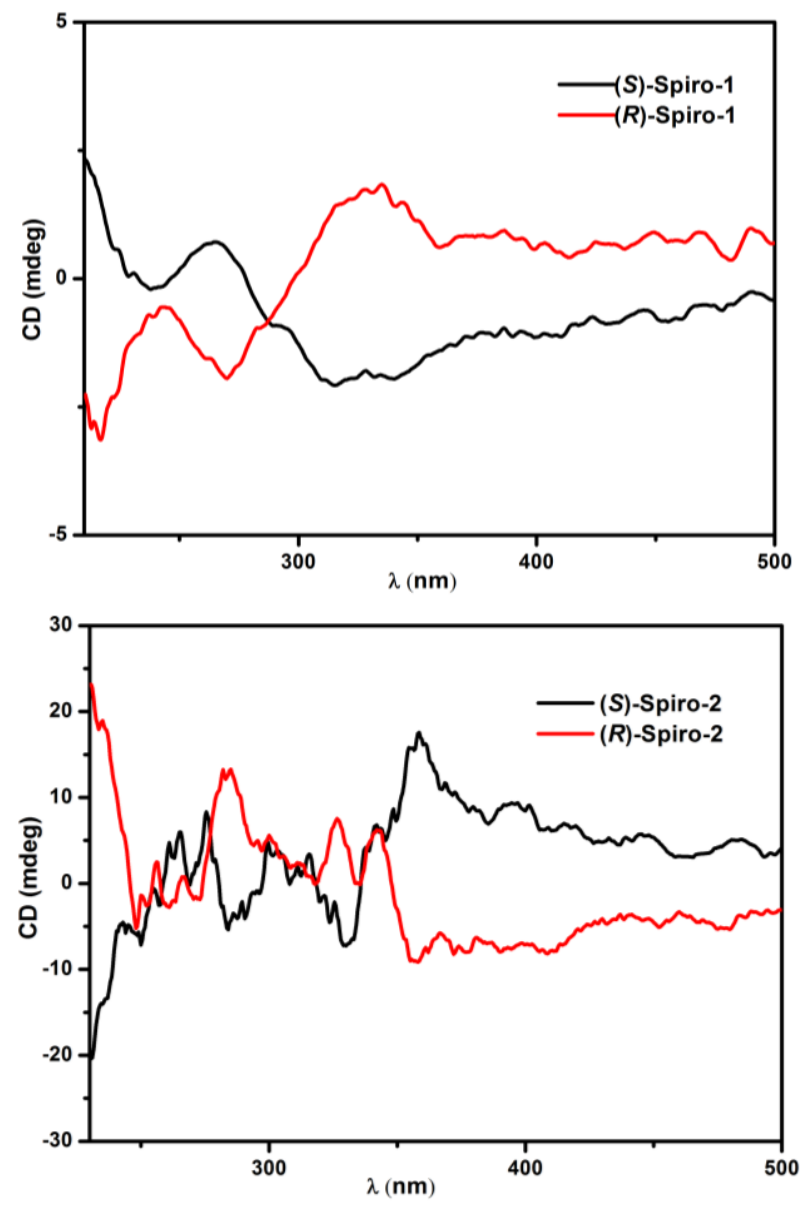

9. Figure S5. TGA curves

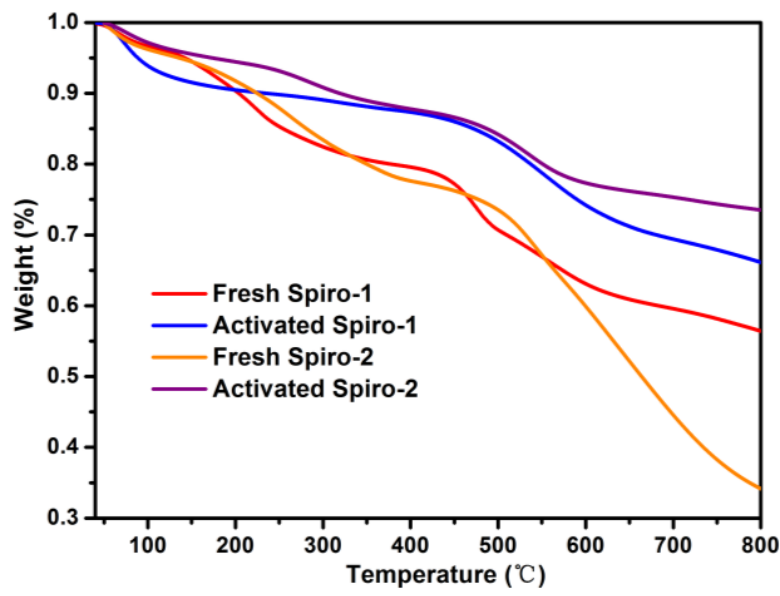




\section{Figure S6. IR spectra}

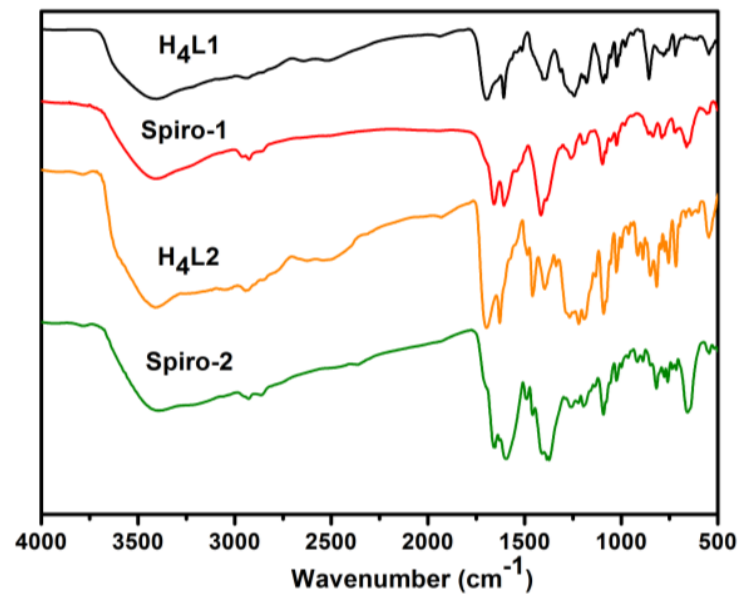

11. Figure S7. Single crystal diffraction study of Spiro-2 after different treatment
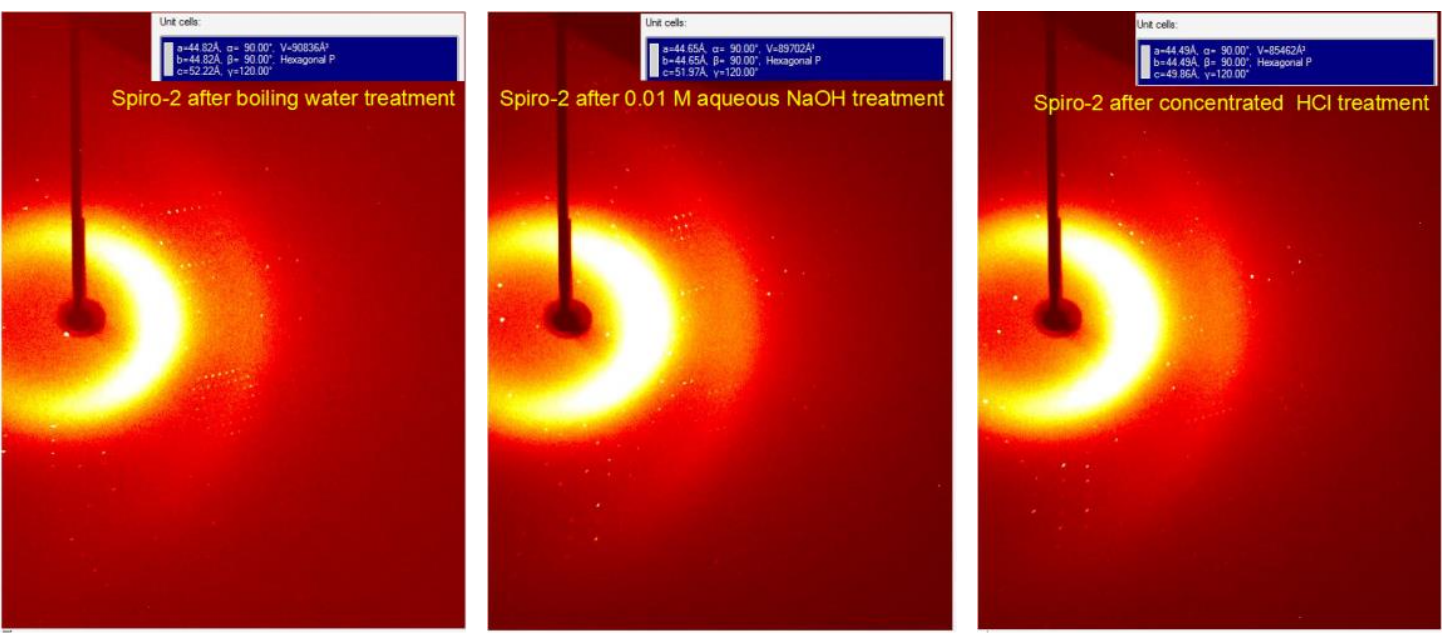

\section{Figure S8. Molecular mechanics simulation of large substrate}

The space-filling models were calculated by Materials Studio (7.0), and were fully optimized using MS Forcite molecular dynamics module (universal force fields, Ewald summations) method.

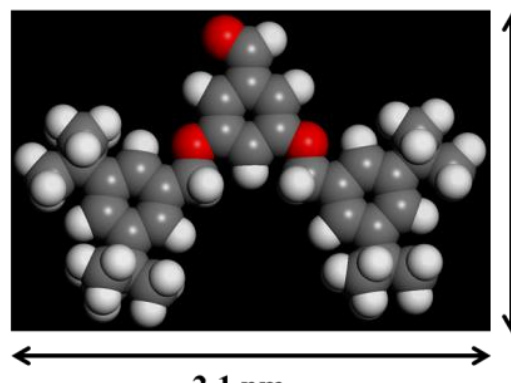<smiles>CC(C)(C)c1cc(COc2cc(C(C)(C)C)cc(C(C)(C)C)c2)cc(OCc2cc(C(C)(C)C)cc(C(C)(C)C)c2)c1</smiles>

$2.1 \mathrm{~nm}$ 


\section{Dye inclusion experiments}

The activated Spiro-1 (15 mg) and Spiro-2 $(15 \mathrm{mg})$ were soaked in a saturated solution of Methyl orange in methanol for $24 \mathrm{~h}$, respectively. To remove the dye adsorbed on the surface of crystals, the resulting colored crystals were washed with methanol until the solution became colorless. The washed samples were then sonicated with methanol several times until the crystals became colorless, the red solution was diluted to $100 \mathrm{~mL}$.

The same procedures were also used for the Rhodamine 6G uptake studies. Absorption experiments were performed on Lambda 20 UV/Vis Spectrometer. The amount of dyes adsorbed were determined by comparing the UV absorptions with the standard curves.

\section{Creation of a standard curve:}

$$
A=\log _{10} \frac{I_{0}}{I_{t}}=\log _{10} \frac{1}{T}=k \cdot l \cdot c
$$

Methyl orange: (1) Methyl orange $(32.73 \mathrm{mg}, 0.1 \mathrm{mmol})$ was added to a flask and diluted to $1000 \mathrm{~mL}$ with methanol. Then, 40,20, 10, 5 and $2.5 \mathrm{~mL}$ of the as-prepared solution were diluted to $100 \mathrm{~mL}$, respectively. (2) The absorbance of different concentrations of Methyl orange was determined by UV/Vis Spectrometer. Data for known concentrations of Methyl orange were used to make the standard curve, plotting concentration on the $\mathrm{X}$ axis, and the assay measurement of absorbance on the $\mathrm{Y}$ axis. According to the Beer-Lambert law, the standard curve can be calculated by linear fitting of the data.

Rhodamine 6G: (1) Rhodamine 6G (47.9 mg) was added to a flask and diluted to $1000 \mathrm{~mL}$ with methanol. Then, $20,10,5,2.5$ and $1.25 \mathrm{~mL}$ of the as-prepared solution were diluted to $100 \mathrm{~mL}$, respectively. (2) The absorbance of different concentrations of Rhodamine 6G was determined by UV/Vis Spectrometer. Data for known concentrations of Rhodamine 6G were used to make the standard curve, plotting concentration on the $\mathrm{X}$ axis, and the assay measurement of absorbance on the $\mathrm{Y}$ axis. According to the Beer-Lambert law, the standard curve can be calculated by linear fitting of the data.

The absorbance of the sample solutions were determined by the UV/Vis Spectrometer. The concentrations of dye can be calculated by comparing the UV-Vis absorbance with a standard curve.
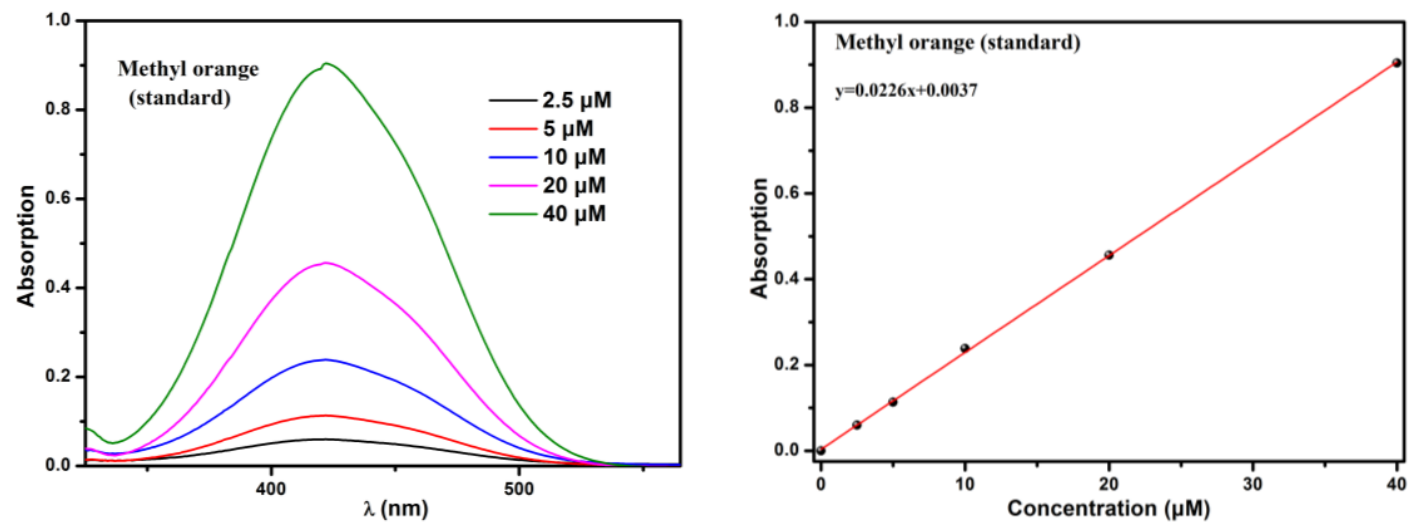

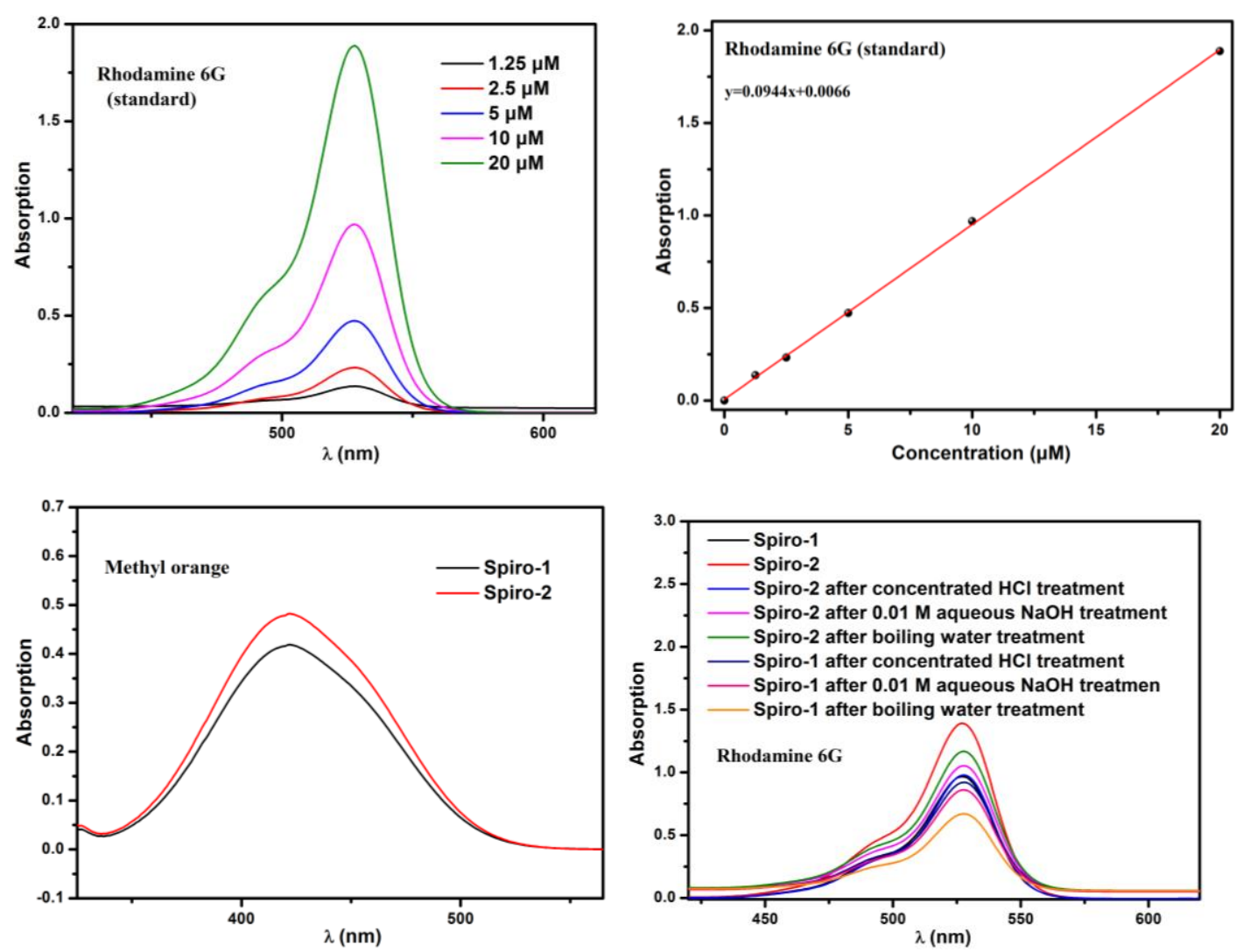

Table S4. Dye adsorption results

\begin{tabular}{|c|c|c|c|c|c|c|c|c|}
\hline & Spiro-1 & Spiro-2 & $\begin{array}{c}\text { Spiro-1 } \\
\text { (boiling } \\
\text { water })\end{array}$ & $\begin{array}{c}\text { Spiro-1 } \\
(\mathrm{Conc} . \\
\mathrm{HCl})\end{array}$ & $\begin{array}{c}\text { Spiro-1 } \\
(0.01 \mathrm{M} \\
\mathrm{NaOH})\end{array}$ & $\begin{array}{c}\text { Spiro-2 } \\
\text { (boiling } \\
\text { water })\end{array}$ & $\begin{array}{c}\text { Spiro-2 } \\
(\mathrm{Conc} . \\
\mathrm{HCl})\end{array}$ & $\begin{array}{c}\text { Spiro-2 } \\
(0.01 \mathrm{M} \\
\mathrm{NaOH})\end{array}$ \\
\hline $\begin{array}{c}\text { Methyl } \\
\text { orange }\end{array}$ & 4.3 & 5.9 & - & - & - & - & - & - \\
\hline $\begin{array}{c}\text { Rhodamine } \\
6 \mathrm{G}\end{array}$ & 2.4 & 4.1 & 2.5 & 2.2 & 2.0 & 3.4 & 3.1 & 2.9 \\
\hline
\end{tabular}




\section{Additional catalytic results}

14.1. Figure S9. Recycle experiment for Spiro-1 catalyzed acetalization reaction
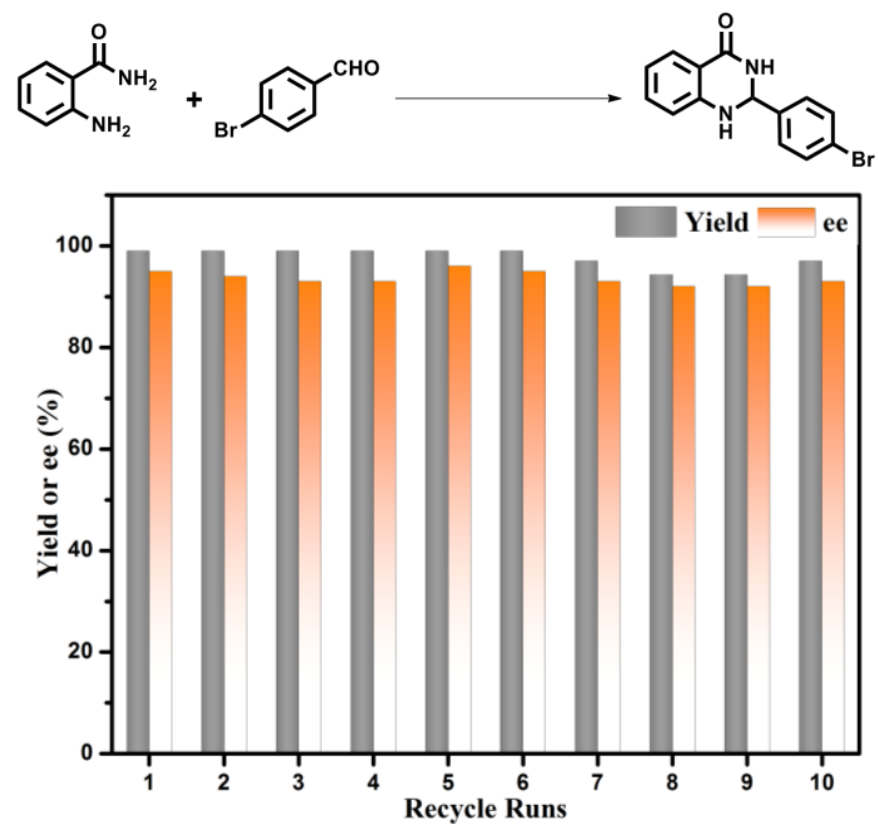

14.2. Figure S10. Recycle experiment for Spiro-1 catalyzed two-component Friedel-Crafts reaction
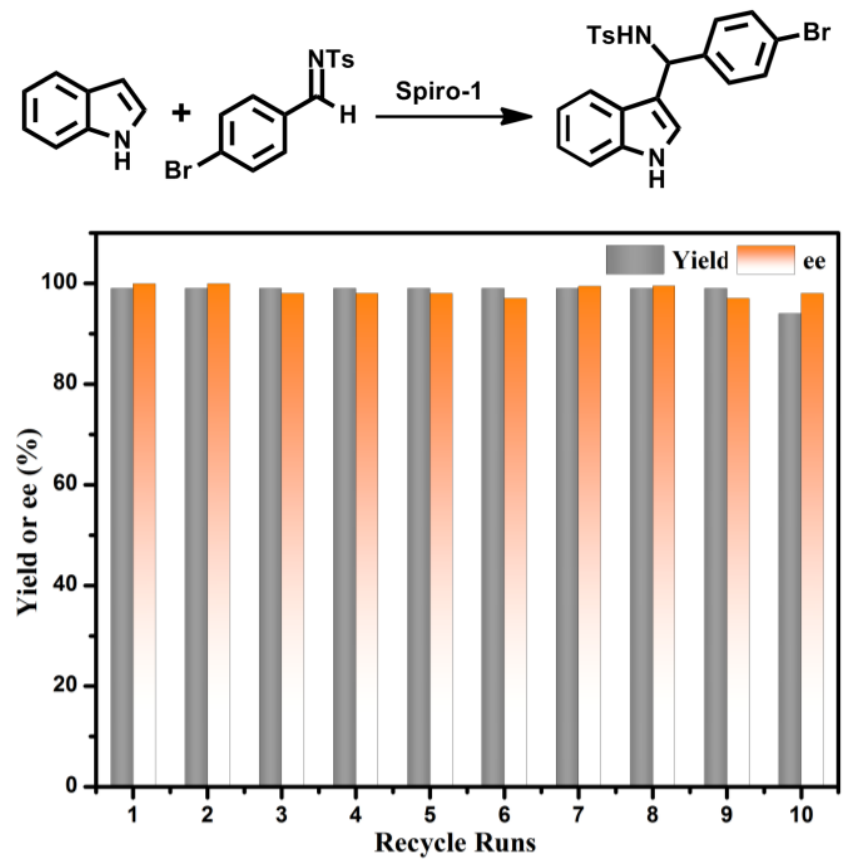
14.3. Figure S11. Recycle experiment for Spiro-1 catalyzed iso-Pictet-Spengler Reaction
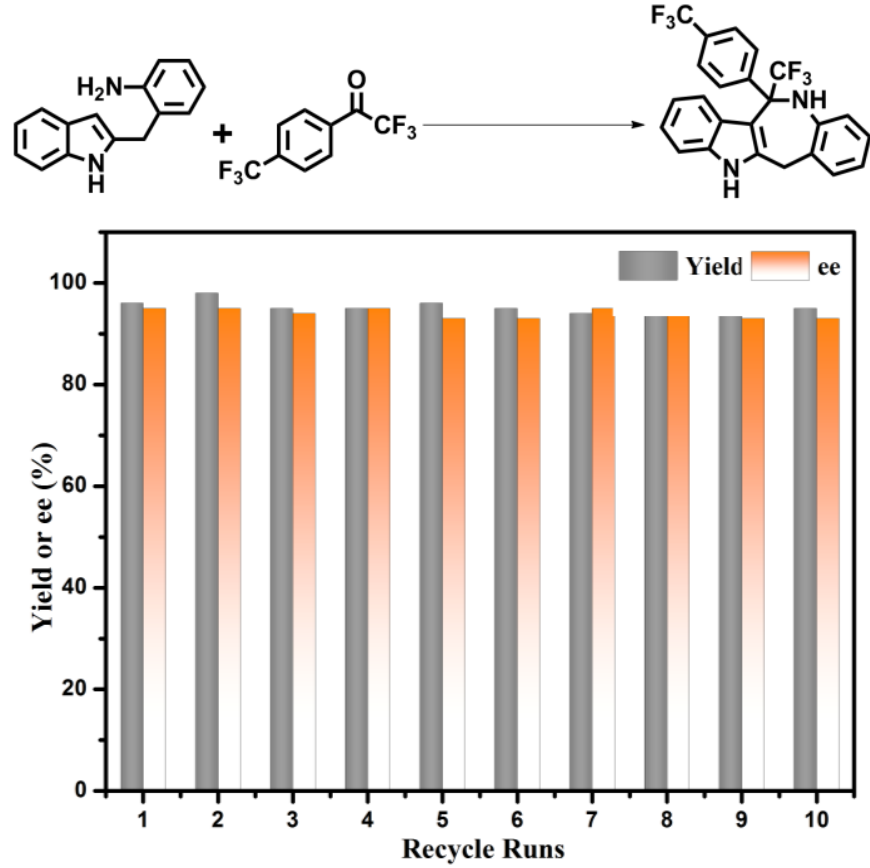

14.4. Table S5. Optimization of the reaction conditions for acetalization reaction

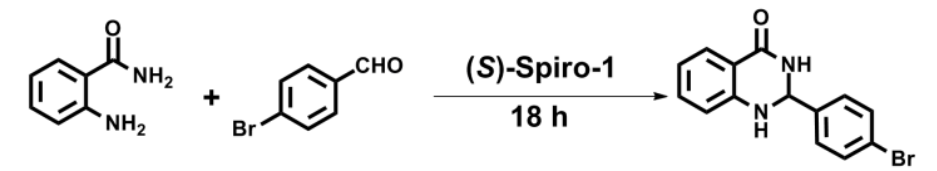

\begin{tabular}{ccccccc}
\hline entry & solvent & loading $(\mathrm{mol} \%)$ & $\mathrm{T}\left({ }^{\circ} \mathrm{C}\right)$ & additive & yield $(\%)^{a}$ & ee $(\%)^{b}$ \\
\hline 1 & $\mathrm{CHCl}_{3}$ & 5 & $\mathrm{rt}$ & $4 \AA \mathrm{MS}$ & $\mathrm{n} . \mathrm{r}$. & $\mathrm{n} . \mathrm{d}$. \\
2 & $\mathrm{CHCl}_{3}$ & 5 & 40 & $4 \AA \mathrm{MS}$ & 52 & 81 \\
3 & $\mathrm{CHCl}_{3}$ & 5 & 60 & $4 \AA \mathrm{MS}$ & 89 & 87 \\
4 & $\mathrm{CHCl}_{3}$ & 5 & 80 & $4 \AA \mathrm{MS}$ & 95 & 77 \\
5 & $\mathrm{THF}$ & 5 & 60 & $4 \AA \mathrm{MS}$ & 91 & 87 \\
6 & toluene & 5 & 60 & $4 \AA \mathrm{MS}$ & 83 & 64 \\
7 & $\mathrm{MeCN}$ & 5 & 60 & $4 \AA \mathrm{MS}$ & 92 & 73 \\
8 & $\mathrm{DCE}$ & 5 & 60 & $4 \AA \mathrm{MS}$ & 78 & 59 \\
9 & $\mathrm{CHCl}_{3}$ & 5 & 60 & $\mathrm{MgSO}_{4}$ & 99 & 96 \\
10 & $\mathrm{CHCl}_{3}$ & 5 & 40 & $\mathrm{MgSO}_{4}$ & 66 & 91 \\
11 & $\mathrm{CHCl}_{3}$ & 2.5 & 60 & $\mathrm{MgSO}_{4}$ & 99 & 96 \\
12 & $\mathrm{CHCl}_{3}$ & 1.25 & 60 & $\mathrm{MgSO}_{4}$ & 94 & 95 \\
13 & $\mathrm{CHCl}_{3}$ & 1.4 & 60 & $\mathrm{MgSO}_{4}$ & 99 & 96 \\
\hline
\end{tabular}

${ }^{a}$ Isolated yield; ${ }^{b}$ Determined by HPLC 
14.5. Table S6. Optimization of the reaction conditions for Friedel-Crafts reaction

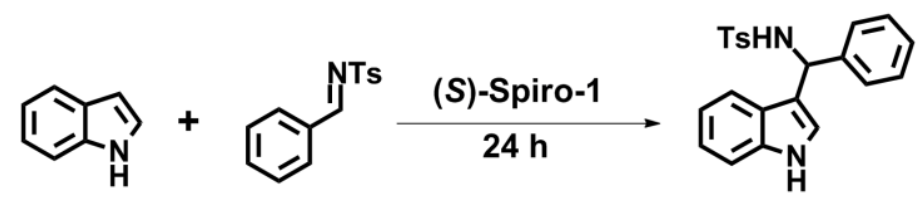

\begin{tabular}{clcccc}
\hline entry & solvent & loading $(\mathrm{mol} \%)$ & $\mathrm{T}\left({ }^{\circ} \mathrm{C}\right)$ & yield $(\%)^{a}$ & ee $(\%)^{b}$ \\
\hline 1 & $\mathrm{CHCl}_{3}$ & 5 & $\mathrm{rt}$ & 84 & 92 \\
2 & $\mathrm{CHCl}_{3}$ & 5 & 40 & 98 & 92 \\
3 & $\mathrm{DCE}$ & 5 & 40 & 98 & 99 \\
4 & $\mathrm{DCE}$ & 2.5 & 40 & 98 & 99 \\
5 & $\mathrm{DCE}$ & 1.25 & 40 & 91 & 97 \\
6 & $\mathrm{DCE}$ & 1.4 & 40 & 98 & 99 \\
\hline
\end{tabular}

${ }^{a}$ Isolated yield; ${ }^{b}$ Determined by HPLC

14.6. Table S7. Optimization of the reaction conditions for iso-Pictet-Spengler reaction

\begin{tabular}{|c|c|c|c|c|c|c|}
\hline entry & solvent & loading (mol\%) & $\mathrm{T}\left({ }^{\circ} \mathrm{C}\right)$ & additive & yield $(\%)^{a}$ & ee $(\%)^{b}$ \\
\hline 1 & $\mathrm{CHCl}_{3}$ & 5 & $\mathrm{rt}$ & $4 \AA \AA M S$ & n.r. & n.d. \\
\hline 2 & $\mathrm{CHCl}_{3}$ & 5 & 35 & $4 \AA ̊ M S$ & n.r. & n.d. \\
\hline 3 & $\mathrm{CHCl}_{3}$ & 5 & 60 & $4 \AA ̊ M S$ & 69 & 78 \\
\hline 4 & $\mathrm{CHCl}_{3}$ & 5 & 80 & $4 \AA ̊ M S$ & 92 & 69 \\
\hline 5 & THF & 5 & 80 & $4 \AA ̊ M S$ & n.r. & n.d. \\
\hline 6 & toluene & 5 & 80 & $4 \AA ̊ M S$ & 96 & 59 \\
\hline 7 & $\mathrm{MeCN}$ & 5 & 80 & $4 \AA ̊ M S$ & n.r. & n.d. \\
\hline 8 & DCE & 5 & 80 & $4 \AA ̊ M S$ & 92 & 64 \\
\hline 9 & $\mathrm{CHCl}_{3}$ & 5 & 60 & $\mathrm{MgSO}_{4}$ & 90 & 90 \\
\hline 10 & $\mathrm{CHCl}_{3}$ & 2.5 & 60 & $\mathrm{MgSO}_{4}$ & 90 & 90 \\
\hline 11 & $\mathrm{CHCl}_{3}$ & 1.25 & 60 & $\mathrm{MgSO}_{4}$ & 76 & 82 \\
\hline 12 & $\mathrm{CHCl}_{3}$ & 2.2 & 60 & $\mathrm{MgSO}_{4}$ & 90 & 90 \\
\hline
\end{tabular}

${ }^{a}$ Isolated yield; ${ }^{b}$ Determined by HPLC 
14.7. Table S8. Catalytic results of arylation of indole ${ }^{a}$

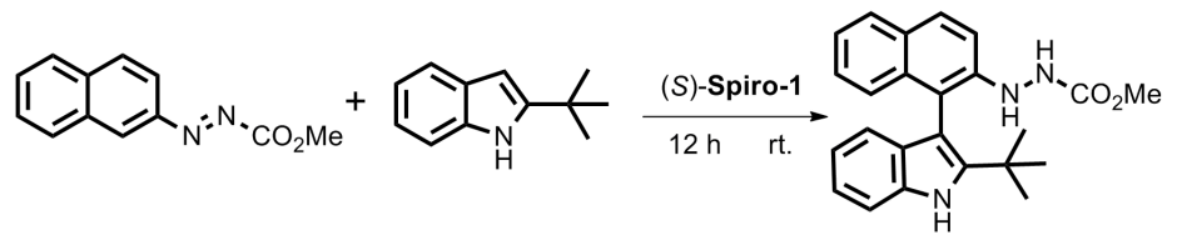

\begin{tabular}{clccc}
\hline entry & solvent & loading $(\mathrm{mol} \%)$ & yield $(\%)^{b}$ & ee $(\%)^{c}$ \\
\hline 1 & $\mathrm{DCM}$ & 1.4 & 91 & 81 \\
2 & $\mathrm{DCE}$ & 1.4 & 84 & 76 \\
3 & $\mathrm{CHCl}$ & 1.4 & 95 & 85 \\
4 & $\mathrm{MeCN}$ & 1.4 & 83 & 53 \\
5 & $\mathrm{EA}$ & 1.4 & n.r. & n.d. \\
6 & $\mathrm{Ph}-\mathrm{CH}_{3}$ & 1.4 & 92 & 91 \\
$7^{d}$ & $\mathrm{Ph}^{d}-\mathrm{CH}_{3}$ & 1.4 & 90 & 91 \\
8 & $\mathrm{Ph}^{-\mathrm{CH}_{3}}$ & 2.1 & 93 & 91 \\
$9^{d}$ & $\mathrm{Ph}^{d}-\mathrm{CH}_{3}$ & 2.1 & 93 & 91 \\
\hline
\end{tabular}

${ }^{a}$ The reaction was carried out with $0.1 \mathrm{mmol}$ scale in $1 \mathrm{~mL}$ solvent for $12 \mathrm{~h}$ at room temperature, the ratio of (E)-methyl 2-(naphthalen-2-yl) diazenecarboxylate and 2-tert-butylindole was 1:1.2; ${ }^{b}$ Isolated yield; ${ }^{c}$ Determined by HPLC; ${ }^{d}$ Reaction was conduct at $0{ }^{\circ} \mathrm{C}$.

14.8. Table S9. Catalytic results of oxidative dearomatization of naphthol ${ }^{a}$

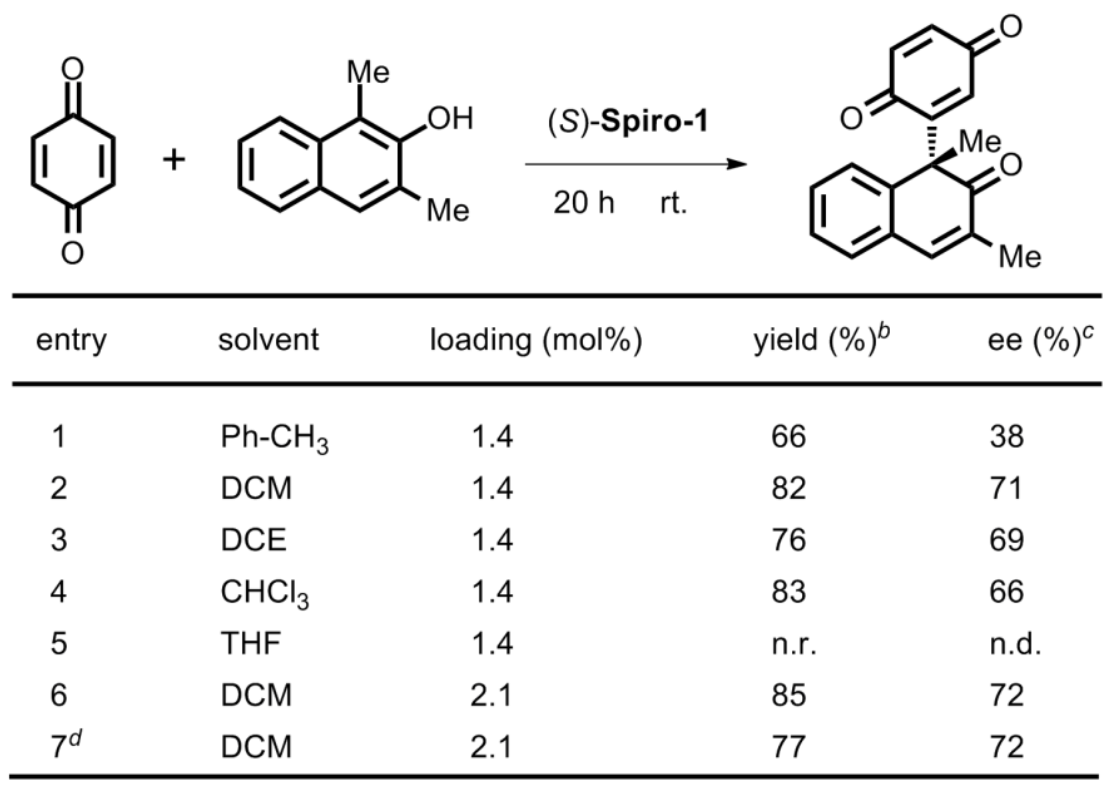

${ }^{a}$ The reaction was carried out with $0.1 \mathrm{mmol}$ scale in $1 \mathrm{~mL}$ solvent for $20 \mathrm{~h}$ at room temperature, the ratio of benzoquinone and 1,3-dimethyl-2-naphthol was $3: 1 ;{ }^{b}$ Isolated yield; ${ }^{c}$ Determined by HPLC; ${ }^{d}$ Reaction was conduct at $0{ }^{\circ} \mathrm{C}$. 
14.9. Table S10. Three-component deacetalization-acetalization of 2 -aminobenzamide with Arylaldehydes Cyclic Acetal ${ }^{a}$

\begin{tabular}{|c|c|c|c|c|}
\hline entry & cat. & $\mathbf{R}$ & yield $(\%)^{b}$ & ee $(\%)^{c}$ \\
\hline 1 & (S)-Spiro-1 & 4-H & 96 & 92 \\
\hline 2 & & $4-B r$ & 93 & 91 \\
\hline 3 & & 4-Me & 87 & 93 \\
\hline 4 & & $4-F$ & 85 & 87 \\
\hline 5 & & $4-\mathrm{NO}_{2}$ & 91 & 83 \\
\hline 6 & & $3-\mathrm{NO}_{2}$ & 93 & 95 \\
\hline 7 & & $3-\mathrm{Cl}$ & 87 & 88 \\
\hline 8 & & $2-\mathrm{NO}_{2}$ & 89 & 93 \\
\hline 9 & $(S)-M_{4} L_{1}$ & 4-H & 81 & 74 \\
\hline 10 & & 4-Br & 80 & 78 \\
\hline 11 & & 4-Me & 83 & 71 \\
\hline 12 & $(S)-S p i r o-2^{d}$ & 4-H & 96 & 84 \\
\hline 13 & & $4-\mathrm{Br}$ & 94 & 84 \\
\hline 14 & & 4-Me & 88 & 78 \\
\hline
\end{tabular}

${ }^{a} \mathrm{CMOFs}$ were activated under vacum at $220{ }^{\circ} \mathrm{C}$, catalyst loading based on 2-aminobenzamide. ${ }^{b}$ Isolated yield. ${ }^{c}$ Determined by HPLC. The absolute configurations of the products were assigned as $S$ by comparing their HPLC profiles with those reported in literature. ${ }^{d}$ Reaction time: $15 \mathrm{~h}$.

14.10. Table S11. Comparison of catalytic results

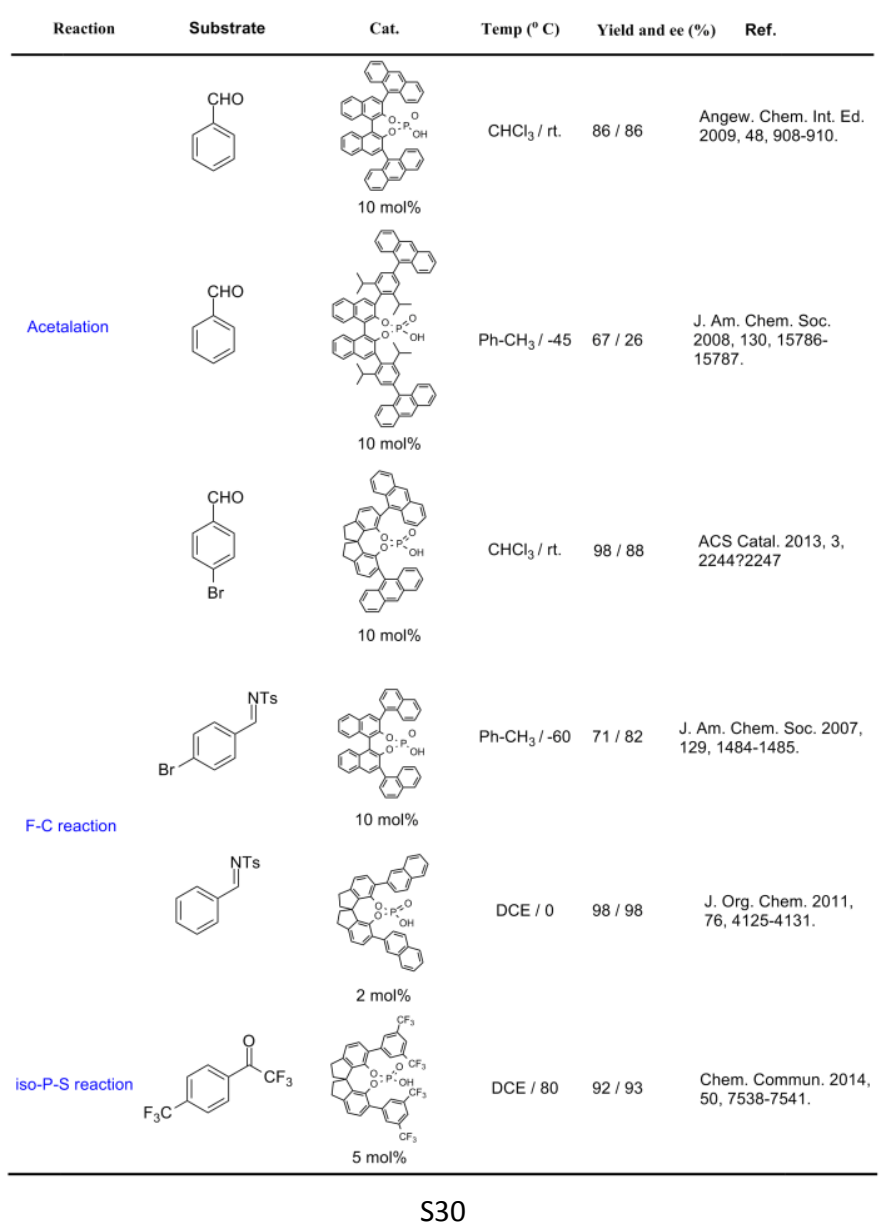


15. Figure S12. ${ }^{31} \mathrm{P}$ NMR spectra of the mixture of $\mathrm{ZrOCl}_{2}$ and $\mathrm{Me}_{4} \mathrm{~L}_{1}$

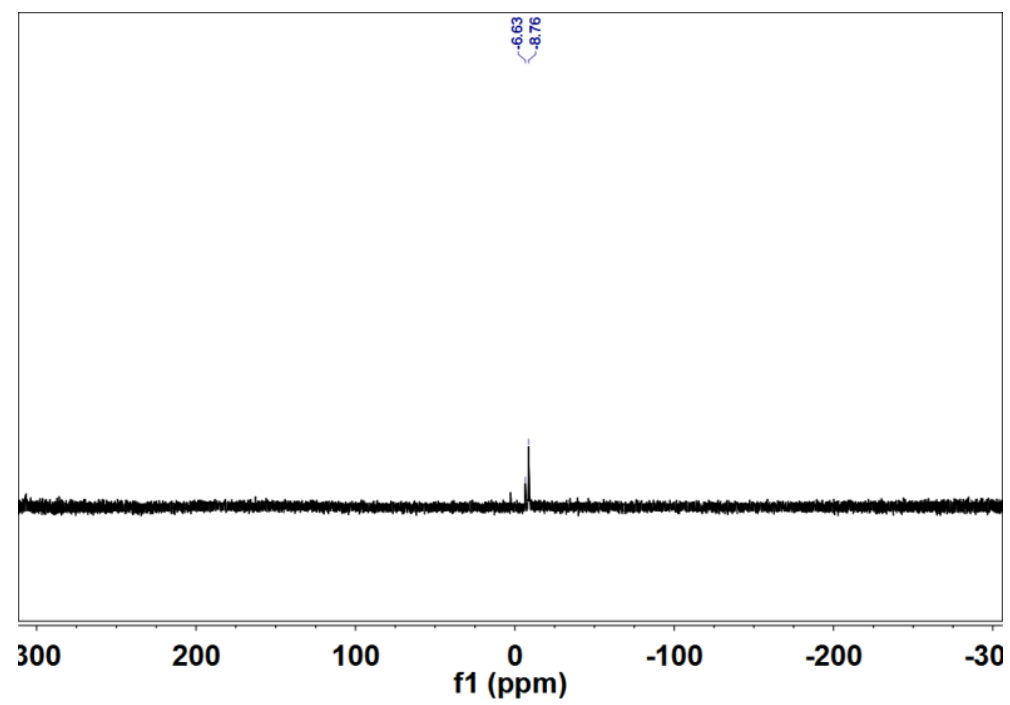

16. Table S12. Comparison of $S_{B E T}$ and total solvent-accessible volume of reported highly porous MOFs.

\begin{tabular}{|c|c|c|c|}
\hline $\begin{array}{c}\text { Highly Porous } \\
\text { Zr-MOFs }\end{array}$ & $S_{\mathrm{BET}}\left(\mathrm{m}^{2} / \mathrm{g}\right)$ & $\begin{array}{c}\text { Total solvent-accessible } \\
\text { volumn }\end{array}$ & Ref. \\
\hline DUT-68 & 1786 & 60.2 & $\begin{array}{c}\text { Cryst. Growth Des. } \\
\mathbf{2 0 1 3}, 13,1231\end{array}$ \\
\hline DUT-67 & 1767 & 66.1 & $\begin{array}{c}\text { Cryst. Growth Des. } \\
\mathbf{2 0 1 3}, 13,1231\end{array}$ \\
\hline UiO-66 & 1290 & 51.8 & $\begin{array}{l}\text { J. Am. Chem. Soc. } \\
\text { 2008, 130, } 13850 .\end{array}$ \\
\hline UiO-68 & 3738 & 74.8 & $\begin{array}{l}\text { J. Am. Chem. Soc. } \\
\text { 2008, } 130,13850 .\end{array}$ \\
\hline NU-1000 & 2320 & 79.4 & $\begin{array}{l}\text { J. Am.Chem. Soc. } \\
\text { 2013, 135, } 10294 .\end{array}$ \\
\hline NU-1100 & 4020 & 78.1 & $\begin{array}{c}\text { Chem. Eur. J. 2014, } \\
\text { 20, } 12389 .\end{array}$ \\
\hline PCN-221 & 650 & 70.5 & $\begin{array}{c}\text { Inorg. Chem. 2013, } \\
52,12661\end{array}$ \\
\hline PCN-222 & 2200 & 79.4 & $\begin{array}{l}\text { Angew. Chem. Int. } \\
\text { Ed. 2012, 51, } 10307\end{array}$ \\
\hline PCN-224 & 2600 & 78.9 & $\begin{array}{l}\text { J. Am. Chem. Soc. } \\
\text { 2013, 135, } 17105\end{array}$ \\
\hline
\end{tabular}




\begin{tabular}{|c|c|c|c|}
\hline NU-1101 & 4422 & 53.9 & $\begin{array}{c}\text { J. Am. Chem. Soc. } \\
\text { 2015, 137, } 3585\end{array}$ \\
\hline NU-1102 & 4712 & 78.8 & $\begin{array}{c}\text { J. Am. Chem. Soc. } \\
\text { 2015, 137, } 3585\end{array}$ \\
\hline NU-1103 & 5646 & 74.1 & $\begin{array}{c}\text { J. Am. Chem. Soc. } \\
\text { 2015, 137, } 3585 .\end{array}$ \\
\hline NU-1104 & 5290 & 78.2 & $\begin{array}{c}\text { J. Am. Chem. Soc. } \\
\mathbf{2 0 1 5}, 137,3585 .\end{array}$ \\
\hline PCN-777 & 2008 & 71.2 & $\begin{array}{l}\text { Angew. Chem. Int. } \\
\text { Ed. 2015, 54, } 149 .\end{array}$ \\
\hline BUT-12 & 2867 & 79.1 & $\begin{array}{c}\text { J. Am. Chem. Soc. } \\
\text { 2016, 138, } 6204\end{array}$ \\
\hline BUT-13 & 2724 & 84.6 & $\begin{array}{c}\text { J. Am. Chem. Soc. } \\
\text { 2016, 138, } 6204\end{array}$ \\
\hline BUT-14 & 3595 & 78.2 & $\begin{array}{c}\text { ACS Appl. Mater. } \\
\text { Interfaces. 2017, 9, } \\
10286\end{array}$ \\
\hline BUT-15 & 3590 & 77.6 & $\begin{array}{c}\text { ACS Appl. Mater. } \\
\text { Interfaces. 2017, 9, } \\
10286\end{array}$ \\
\hline BUT-39 & 1812 & 70.1 & $\begin{array}{c}\text { ACS Appl. Mater. } \\
\text { Interfaces. 2018, 10, } \\
16650\end{array}$ \\
\hline PCN-225 & 1902 & 68.1 & $\begin{array}{l}\text { J. Am. Chem. Soc. } \\
\mathbf{2 0 1 3}, 135,13934\end{array}$ \\
\hline pbz-MOF-1 & 2432 & 69 & $\begin{array}{l}\text { J. Am. Chem. Soc. } \\
\text { 2016, 138, } 12767\end{array}$ \\
\hline PCN-94 & 3377 & 64.1 & $\begin{array}{l}\text { J. Am. Chem. Soc. } \\
\text { 2014, 136, } 8269\end{array}$ \\
\hline NPF-200 & 5463 & 81.6 & $\begin{array}{l}\text { J. Am. Chem. Soc. } \\
\text { 2016, 138, } 8380\end{array}$ \\
\hline NPF-201 & 534 & 80.0 & $\begin{array}{c}\text { J. Am. Chem. Soc. } \\
\text { 2016, 138, } 8380\end{array}$ \\
\hline MOF-808 & 2060 & 41.3 & $\begin{array}{l}\text { J. Am. Chem. Soc. } \\
\text { 2014, 136, } 4369\end{array}$ \\
\hline MOF-806 & 2220 & 56.5 & $\begin{array}{l}\text { J. Am. Chem. Soc. } \\
\text { 2014, 136, } 4369\end{array}$ \\
\hline DUT-51 & 1859 & 78.0 & $\begin{array}{l}\text { Chem. Commun. } \\
\text { 2012, 48, } 8407\end{array}$ \\
\hline ZJNU-30 & 1570 & 63.8 & Chem. Eur. J. 2016, \\
\hline
\end{tabular}




\begin{tabular}{|c|c|c|c|}
\hline & & & 22,1 \\
\hline Spiro-1 & 2002 & 73.2 & This work \\
\hline Spiro-2 & ND & 91.7 & This work \\
\hline \multicolumn{4}{|c|}{$\begin{array}{c}\text { Other Highly Porous } \\
\text { MOFs }\end{array}$} \\
\hline DUT-60 & 7839 & 90.3 & $\begin{array}{c}\text { Angew. Chem. Int. } \\
\text { Ed. 2018, 57, } 1\end{array}$ \\
\hline MOF-399 & ND & 94 & $\begin{array}{c}\text { Inorg. Chem. 2011, } \\
50,9147\end{array}$ \\
\hline NU-109 & 7010 & 88.7 & $\begin{array}{l}\text { J. Am. Chem. Soc. } \\
\text { 2012, 134, } 15016\end{array}$ \\
\hline NU-110 & 7140 & 89.0 & $\begin{array}{l}\text { J. Am. Chem. Soc. } \\
\text { 2012, 134, } 15016\end{array}$ \\
\hline NU-100 & 6143 & 86.2 & $\begin{array}{c}\text { Nat. Chem. 2010. } 2 \text {, } \\
944\end{array}$ \\
\hline MOF-210 & 6240 & 89 & $\begin{array}{c}\text { Science 2010, 329, } \\
424\end{array}$ \\
\hline MOF-180 & ND & 89 & $\begin{array}{c}\text { Science 2010, 329 } \\
424\end{array}$ \\
\hline MOF-200 & 4530 & 90 & $\begin{array}{c}\text { Science 2010, 329 } \\
424\end{array}$ \\
\hline MOF-205 & 4460 & 85 & $\begin{array}{c}\text { Science 2010, 329 } \\
424\end{array}$ \\
\hline MOF-5 & 3800 & 79 & $\begin{array}{l}\text { J. Am. Chem. Soc. } \\
\text { 2007, 129, } 14176\end{array}$ \\
\hline MOF-177 & 4500 & 83 & $\begin{array}{l}\text { J. Mater. Chem. } \\
\text { 2007, 17, } 3197 .\end{array}$ \\
\hline UMCM-2 & 5200 & 83 & $\begin{array}{c}\text { J. Am. Chem. Soc. } \\
\text { 2009, 131, } 4184 .\end{array}$ \\
\hline MIL-101 & 4230 & 83 & $\begin{array}{c}\text { Science 2005, 309, } \\
2040 .\end{array}$ \\
\hline
\end{tabular}




\section{Table S13. Hammett indicators and acidity measured by Hammett indicator tests.}

A set of stock Hammett indicator solutions $(0.5 \mathrm{wt} \%)$ was prepared in an inert atmosphere glovebox by dissolving Hammett indicators in anhydrous benzene. Hammett indicator stock solution $(5 \mathrm{~mL})$ was added to $20 \mathrm{mg}$ of each sample in a $10-\mathrm{mL}$ glass vial in the glovebox. The suspension was swirled every $30 \mathrm{mins}$, and after $4 \mathrm{~h}$ the color of the solid was then recorded. The pKa values were obtained from literatures (Chem. Rev. 1995, 95, 559. / J. Am. Chem. Soc. 1932, 54, 2721.)

\begin{tabular}{|c|c|c|c|c|c|c|c|c|}
\hline \multirow[t]{2}{*}{ Indicators } & \multicolumn{2}{|c|}{ Color } & \multirow[t]{2}{*}{$\mathrm{p} K \mathrm{a}$} & \multicolumn{5}{|c|}{ Hammett Indicator Tests $^{a}$} \\
\hline & $\begin{array}{l}\text { Acid } \\
\text { Form }\end{array}$ & $\begin{array}{l}\text { Base } \\
\text { Form }\end{array}$ & & Spiro-1 & \multicolumn{2}{|c|}{ Spiro-2 } & $\begin{array}{c}\mathrm{Me}_{4} \mathbf{L}_{1} \\
\text { (crystalline) }\end{array}$ & $\begin{array}{c}\mathrm{Me}_{4} \mathbf{L}_{2} \\
\text { (crystalline) }\end{array}$ \\
\hline Neutral red & Red & Yellow & +6.8 & + & \multicolumn{2}{|l|}{+} & + & + \\
\hline Acid red & Red & Yellow & +4.8 & + & \multicolumn{2}{|c|}{+} & + & + \\
\hline 4-Benzeneazo-1-naphthylamine & Red & Yellow & +4.0 & + & \multicolumn{2}{|l|}{+} & + & + \\
\hline Dimethylaminoazobenzene & Red & Yellow & +3.3 & + & \multicolumn{2}{|l|}{+} & + & + \\
\hline 4-Phenylazoaniline & Red & Orange & +2.8 & + & \multicolumn{2}{|l|}{+} & - & - \\
\hline 4-Phenazodiphenylamine & Purple & Yellow & +1.5 & + & \multicolumn{2}{|l|}{+} & - & - \\
\hline 2-Nitroaniline & Red & Yellow & -0.2 & + & \multicolumn{2}{|l|}{+} & - & - \\
\hline 4-Nitrodiphenylamine & Red & Yellow & -2.4 & + & \multicolumn{2}{|l|}{-} & - & - \\
\hline Dicinnamlalcetone & Red & Yellow & -3.0 & - & \multicolumn{2}{|l|}{-} & - & - \\
\hline 2,4-Dichloro-6-nitroaniline & Red & Yellow & -3.2 & - & \multicolumn{2}{|l|}{-} & - & - \\
\hline 2,4-Dinitroaniline & Red & Yellow & -4.4 & - & - & & - & - \\
\hline \multirow[t]{2}{*}{ Indicators } & \multicolumn{2}{|c|}{ Color } & $\mathrm{p} K \mathrm{a}$ & \multicolumn{5}{|c|}{ Hammett Indicator Tests $^{a}$} \\
\hline & $\begin{array}{l}\text { Acid } \\
\text { Form }\end{array}$ & $\begin{array}{l}\text { Base } \\
\text { Form }\end{array}$ & & \multicolumn{2}{|c|}{$\begin{array}{c}\text { Spinol-PO } \\
\text { (crystalline) }\end{array}$} & & $\begin{array}{l}\text { ol-PSO } \mathbf{P}_{3} \mathbf{H} \\
\text { ystalline) }\end{array}$ & $\begin{array}{c}\text { Spinol-PS } \mathbf{P}_{2} \mathbf{O}_{2} \mathbf{H} \\
\text { (crystalline) }\end{array}$ \\
\hline Neutral red & Red & Yellow & +6.8 & \multicolumn{2}{|l|}{+} & & + & + \\
\hline Acid red & Red & Yellow & +4.8 & \multicolumn{2}{|l|}{+} & & + & + \\
\hline 4-Benzeneazo-1-naphthylamine & Red & Yellow & +4.0 & \multicolumn{2}{|l|}{+} & & + & + \\
\hline Dimethylaminoazobenzene & Red & Yellow & +3.3 & \multicolumn{2}{|l|}{-} & & + & + \\
\hline 4-Phenylazoaniline & Red & Orange & +2.8 & \multicolumn{2}{|l|}{-} & & + & + \\
\hline 4-Phenazodiphenylamine & Purple & Yellow & +1.5 & \multicolumn{2}{|l|}{-} & & + & + \\
\hline 2-Nitroaniline & Red & Yellow & -0.2 & \multicolumn{2}{|l|}{-} & & + & + \\
\hline 4-Nitrodiphenylamine & Red & Yellow & -2.4 & \multicolumn{2}{|l|}{-} & & - & + \\
\hline Dicinnamlalcetone & Red & Yellow & -3.0 & \multicolumn{2}{|l|}{-} & & - & + \\
\hline 2,4-Dichloro-6-nitroaniline & Red & Yellow & -3.2 & \multicolumn{2}{|l|}{-} & & - & - \\
\hline 2,4-Dinitroaniline & Red & Yellow & -4.4 & \multicolumn{2}{|l|}{-} & & - & - \\
\hline
\end{tabular}

${ }^{a}$ Results of Hammett indicator tests are denoted as color change observed (+) and color change not observed (-). 
18. Figure S13. Kinetic curves of acetalization reaction of 2 -aminobenzamide with 4-bromoaldehyde by using Spiro-1 and Spiro-2 as catalysts

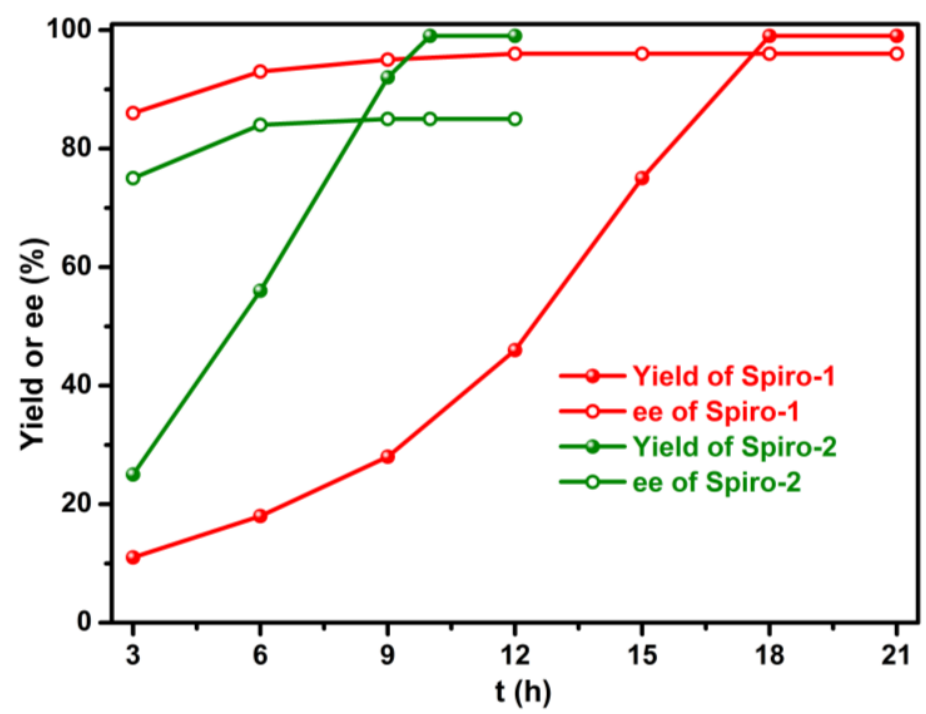

19. Figure S14. Imine intermediate detection in Spiro-1 catalyzed acetalization reaction

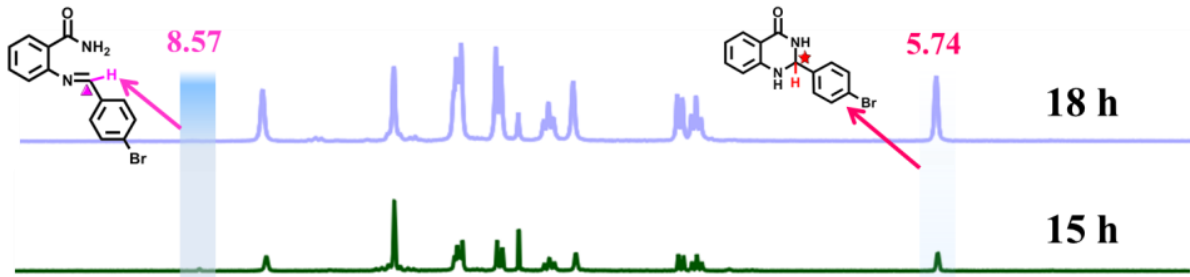

$12 \mathrm{~h}$

$9 \mathrm{~h}$

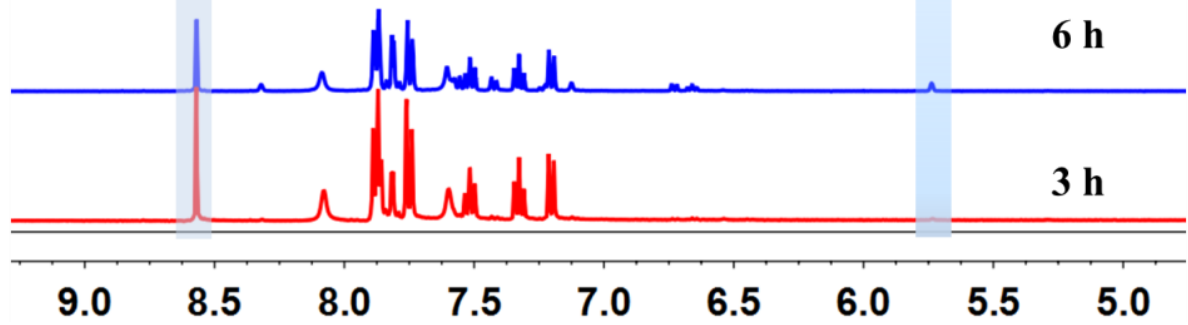


20. HPLC and NMR
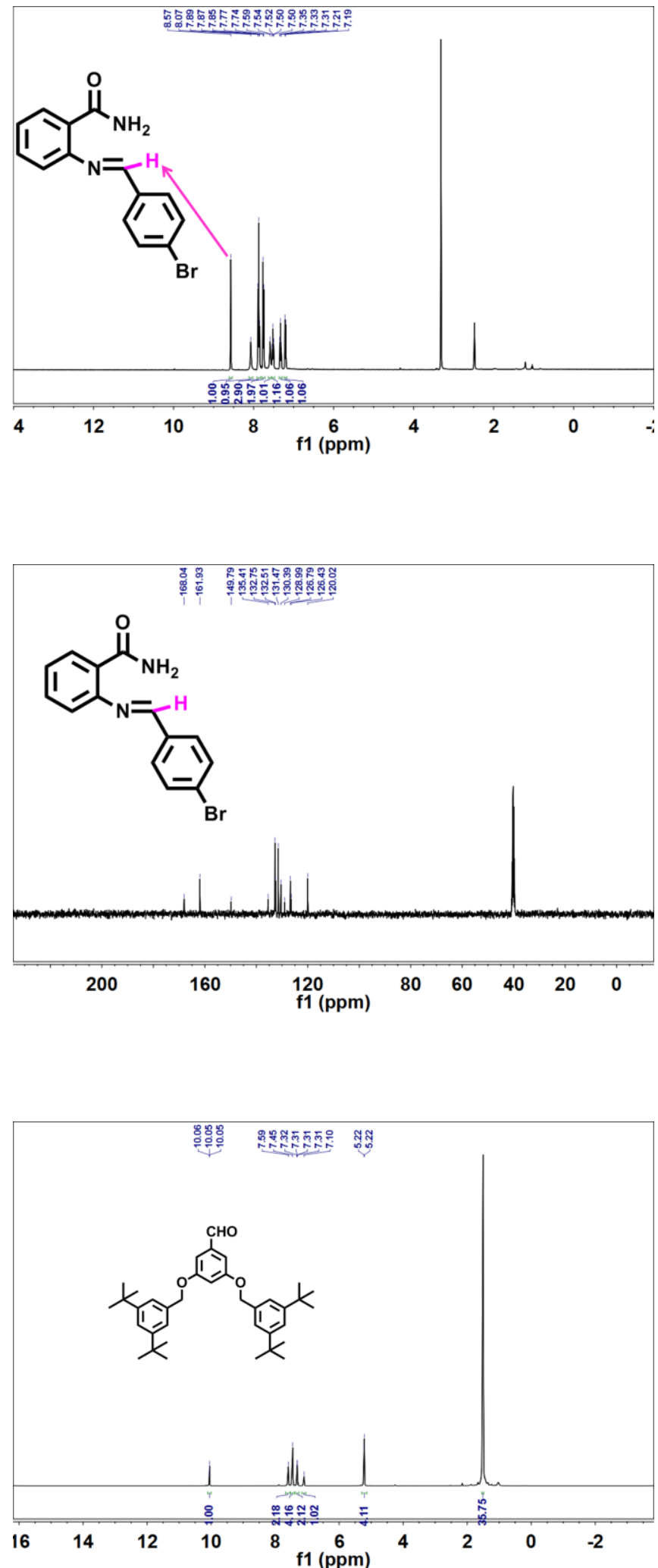

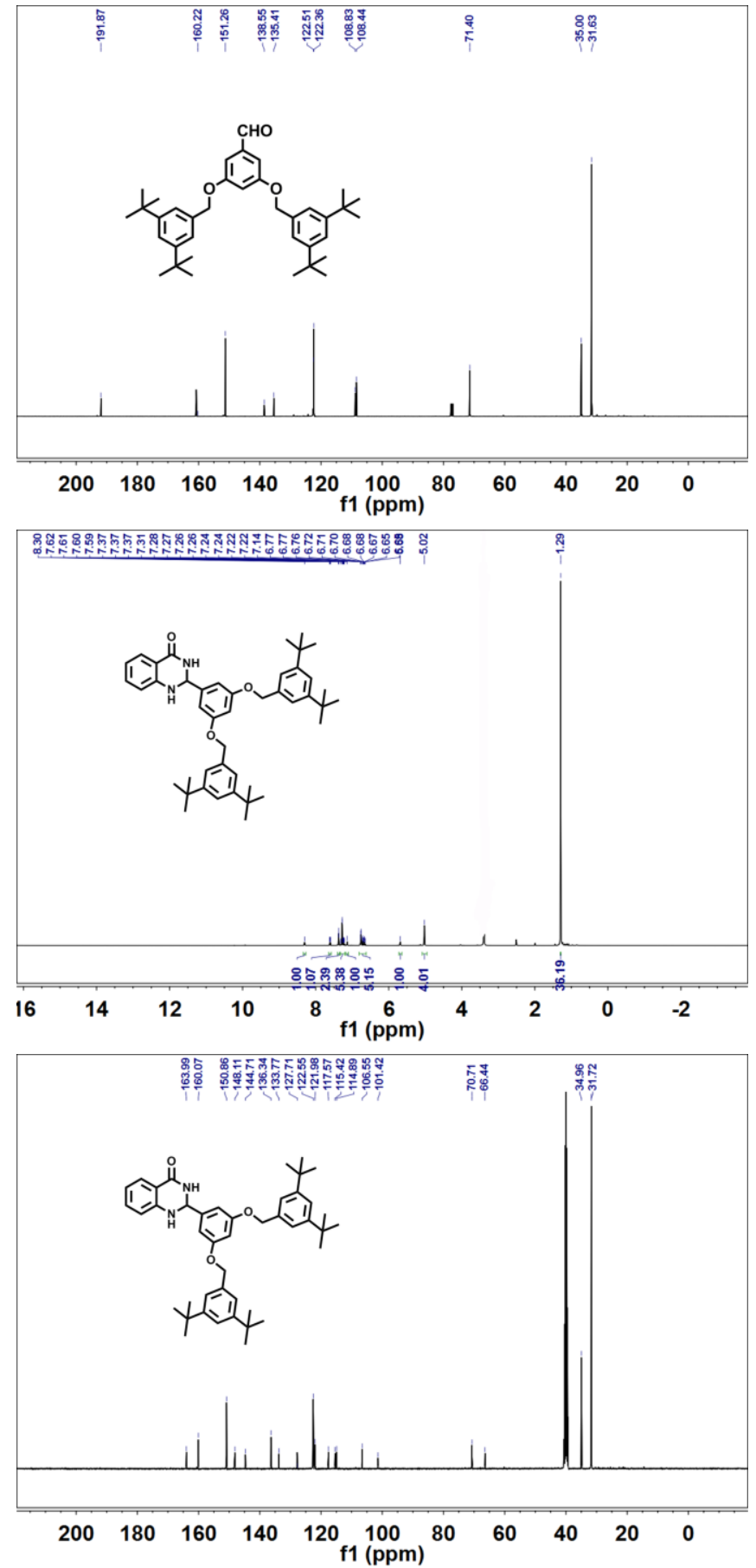

20.1 Acetalization reaction of 2-aminobenzamide with arylaldehydes<smiles>O=C1NC(c2ccccc2)Nc2ccccc21</smiles>

Enantiomeric excess was determined by HPLC with a chiralcel AD-H column (hexane/iPrOH = $80 / 20,1.0 \mathrm{~mL} / \mathrm{min}), \mathrm{t}_{\text {major }}=14.250 \mathrm{~min}, \mathrm{t}_{\text {minor }}=17.349 \mathrm{~min}$; ee $=95 \% .{ }^{1} \mathrm{H} \mathrm{NMR}(400 \mathrm{MHz}$, $d_{6}$-DMSO) $\delta 8.27(\mathrm{~s}, 1 \mathrm{H}), 7.57(\mathrm{~d}, J=7.7 \mathrm{~Hz}, 1 \mathrm{H}), 7.46(\mathrm{~d}, J=7.1 \mathrm{~Hz}, 2 \mathrm{H}), 7.33(\mathrm{ddt}, J=12.6$, 
7.3, 3.7 Hz, 3H), 7.21 (ddd, $J=7.2,4.9,1.5 \mathrm{~Hz}, 1 \mathrm{H}), 7.09$ (s, 1H), $6.76-6.59$ (m, 2H), $5.72(\mathrm{~s}$, $1 \mathrm{H})$.
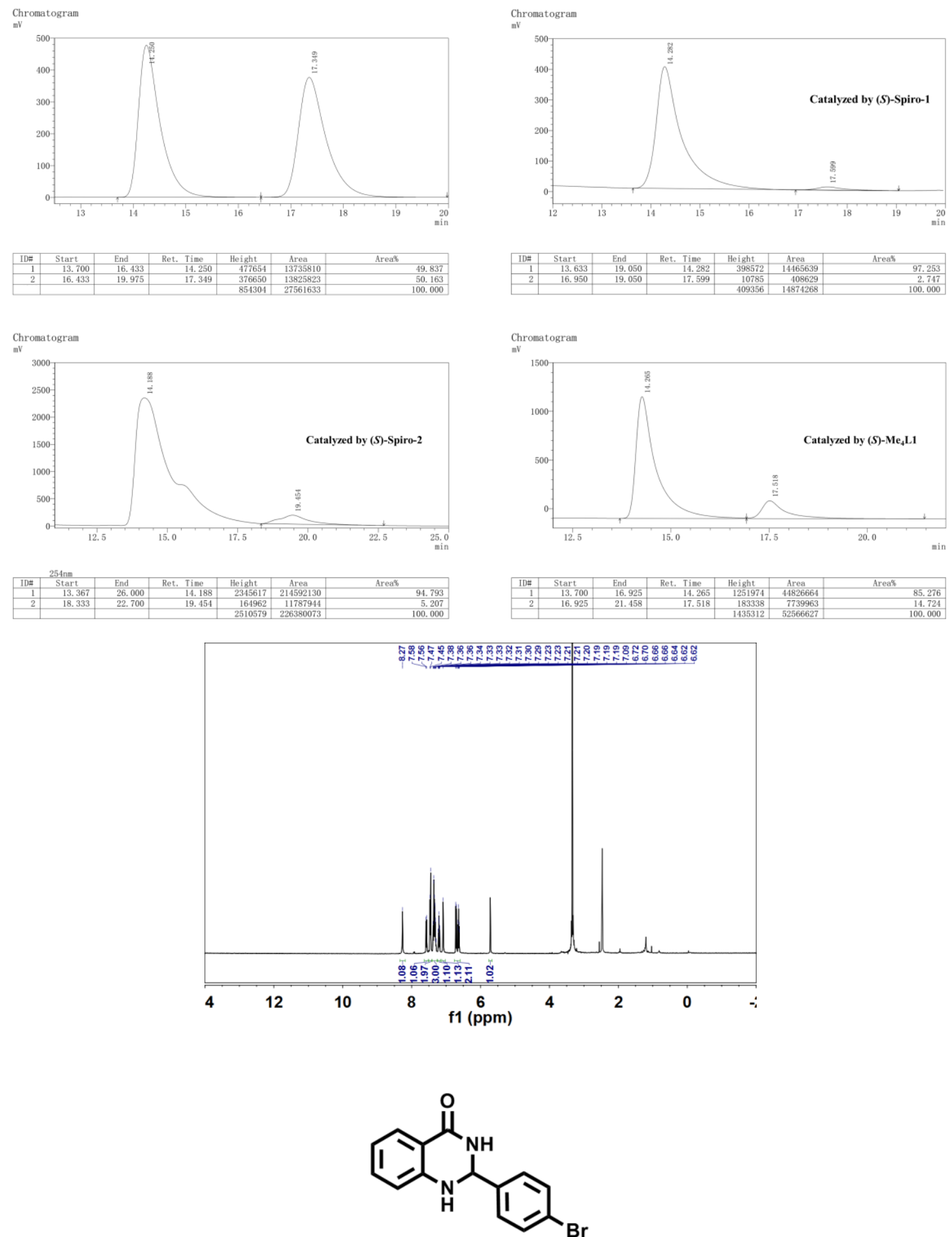

Enantiomeric excess was determined by HPLC with a chiralcel OD-H column (hexane/iPrOH $=$ $80 / 20,1.0 \mathrm{~mL} / \mathrm{min}), \mathrm{t}_{\text {major }}=13.628 \mathrm{~min}, \mathrm{t}_{\text {minor }}=21.299 \mathrm{~min}$; ee $=96 \% .{ }^{1} \mathrm{H} \mathrm{NMR}(400 \mathrm{MHz}$, $d_{6}$-DMSO) $\delta 8.31(\mathrm{~s}, 1 \mathrm{H}), 7.63-7.52(\mathrm{~m}, 3 \mathrm{H}), 7.42(\mathrm{~d}, J=8.4 \mathrm{~Hz}, 2 \mathrm{H}), 7.28-7.18(\mathrm{~m}, 1 \mathrm{H}), 7.12$ (s, 1H), $6.78-6.55(\mathrm{~m}, 2 \mathrm{H}), 5.73(\mathrm{~s}, 1 \mathrm{H})$. 
Chromatogram

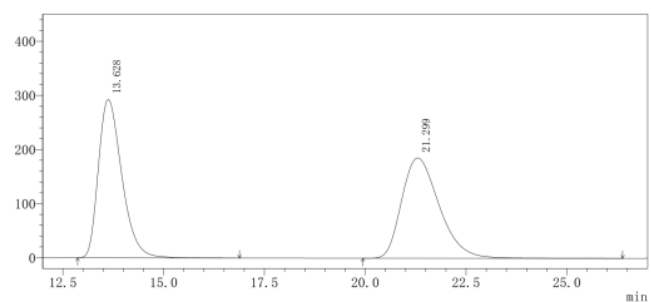

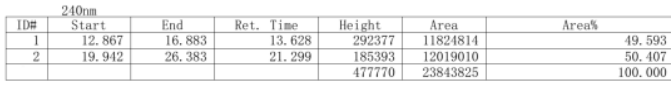

Chromatogram

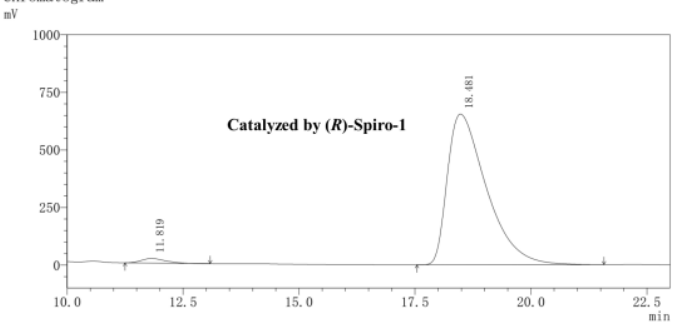

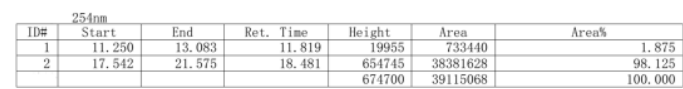

Chromatogram
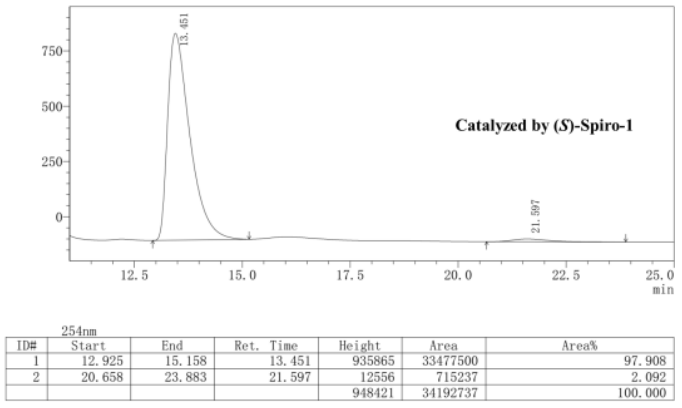

Chromatogram

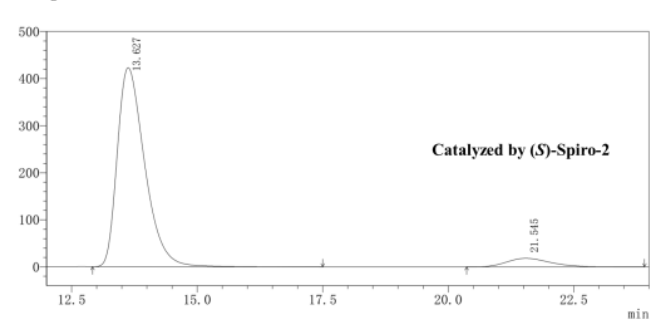

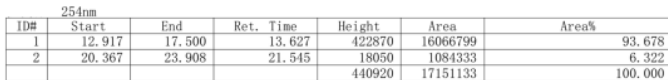
Chromatogra

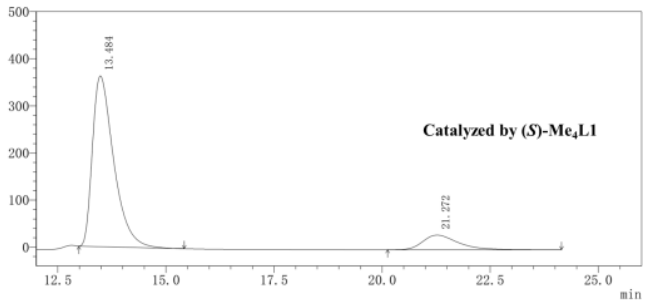

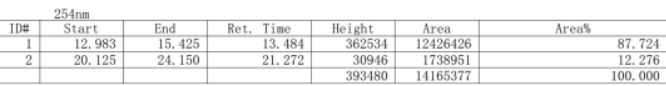

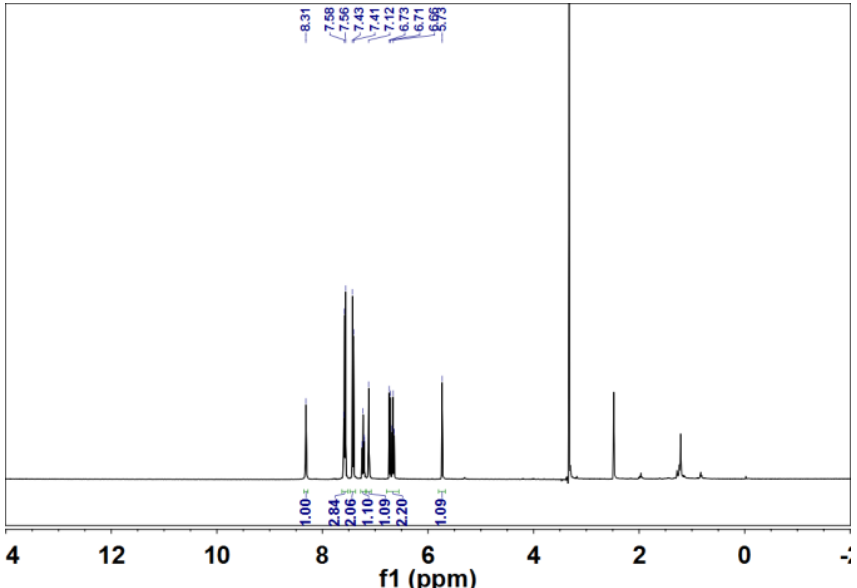




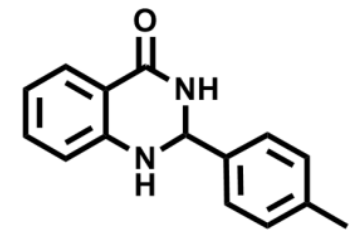

Enantiomeric excess was determined by HPLC with a chiralcel AD-H column (hexane/iPrOH = $85 / 15,1.0 \mathrm{~mL} / \mathrm{min}), \mathrm{t}_{\text {major }}=19.718 \mathrm{~min}, \mathrm{t}_{\text {minor }}=24.631 \mathrm{~min}$; ee $=97 \% .{ }^{1} \mathrm{H} \mathrm{NMR}(400 \mathrm{MHz}$, $d_{6}$-DMSO) $\delta 8.22(\mathrm{~s}, 1 \mathrm{H}), 7.58(\mathrm{dd}, \mathrm{J}=7.7,1.5 \mathrm{~Hz}, 1 \mathrm{H}), 7.33(\mathrm{t}, \mathrm{J}=8.9 \mathrm{~Hz}, 2 \mathrm{H}), 7.20$ (ddd, $\mathrm{J}=$ $22.2,10.4,4.8 \mathrm{~Hz}, 3 \mathrm{H}), 7.04(\mathrm{~s}, 1 \mathrm{H}), 6.76-6.58(\mathrm{~m}, 2 \mathrm{H}), 5.68(\mathrm{~s}, 1 \mathrm{H}), 2.30-2.23(\mathrm{~m}, 3 \mathrm{H})$.
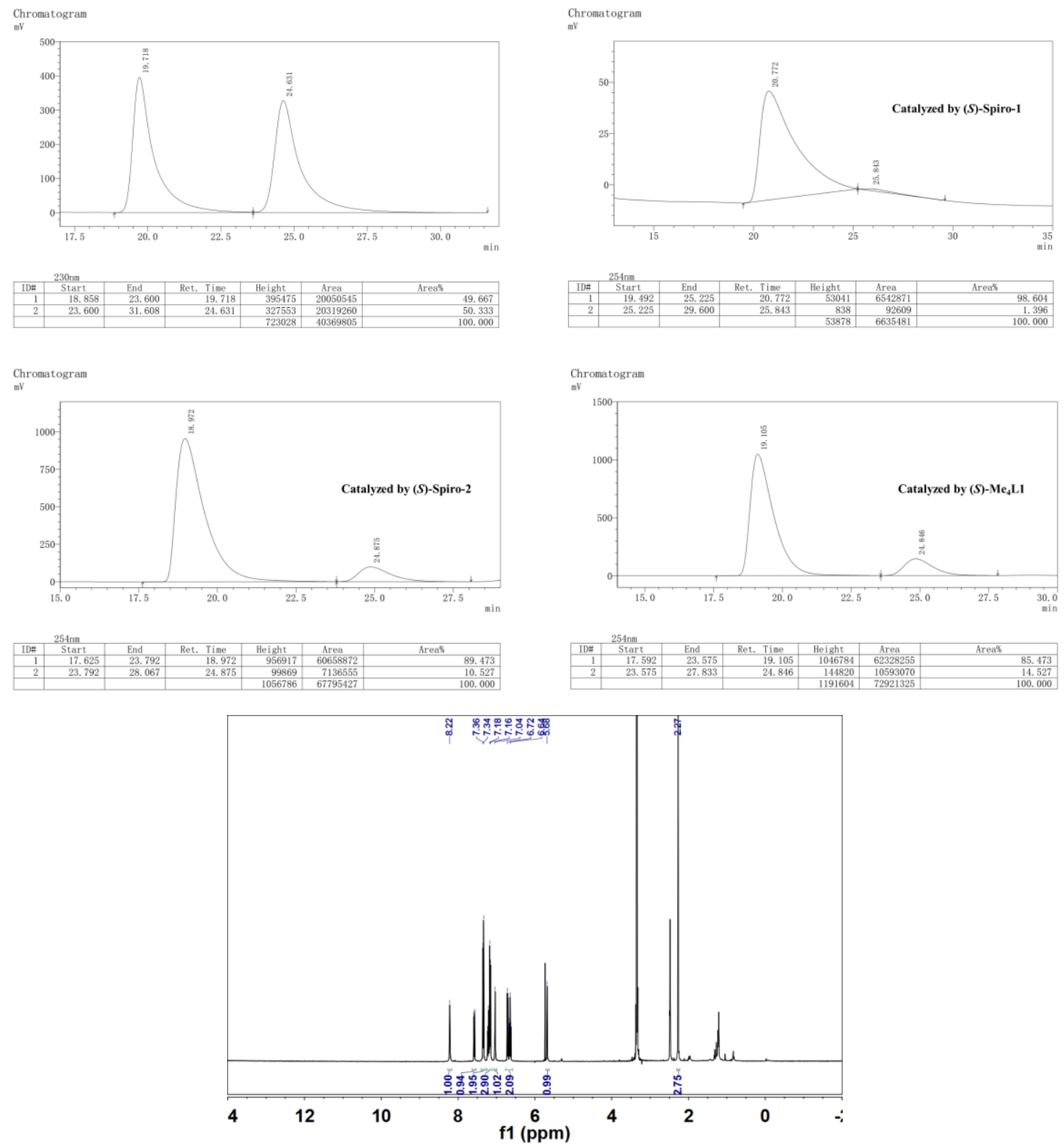


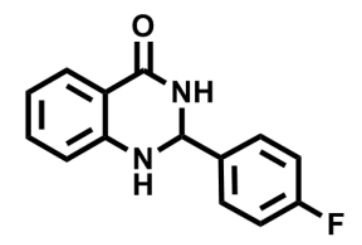

Enantiomeric excess was determined by HPLC with a chiralcel OD-H column (hexane/iPrOH = $80 / 20,1.0 \mathrm{~mL} / \mathrm{min}), \mathrm{t}_{\text {major }}=10.293 \mathrm{~min}, \mathrm{t}_{\text {minor }}=14.807 \mathrm{~min}$; ee $=95 \% .{ }^{1} \mathrm{H} \mathrm{NMR}(400 \mathrm{MHz}$, $d_{6}$-DMSO) $\delta 8.27(\mathrm{~s}, 1 \mathrm{H}), 7.60(\mathrm{dd}, J=7.7,1.4 \mathrm{~Hz}, 1 \mathrm{H}), 7.57-7.48(\mathrm{~m}, 2 \mathrm{H}), 7.29-7.14(\mathrm{~m}, 3 \mathrm{H})$, $7.08(\mathrm{~s}, 1 \mathrm{H}), 6.79-6.60(\mathrm{~m}, 2 \mathrm{H}), 5.76(\mathrm{~s}, 1 \mathrm{H})$.
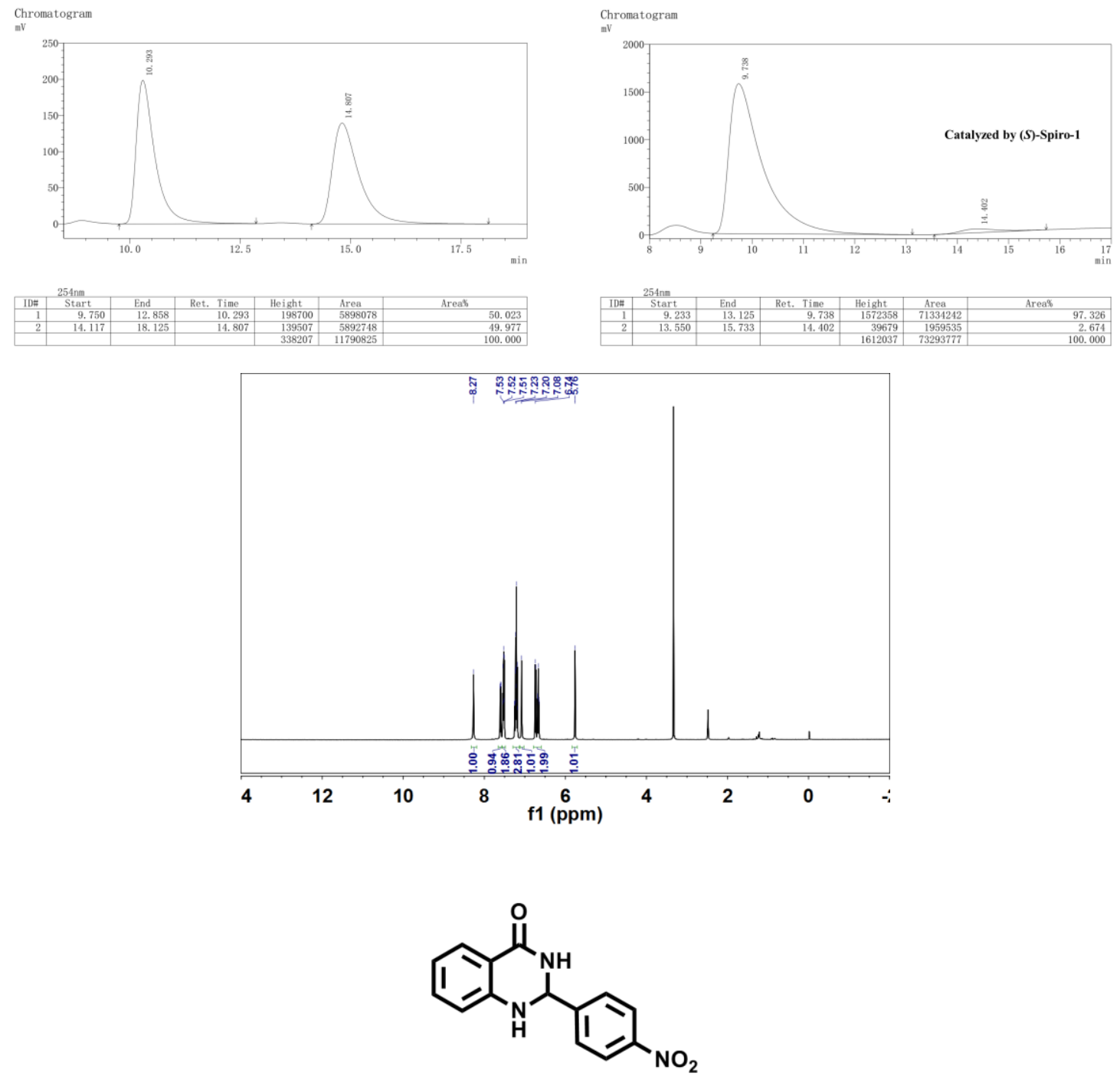

Enantiomeric excess was determined by HPLC with a chiralcel AD-H column (hexane/iPrOH $=$ $85 / 15,1.2 \mathrm{~mL} / \mathrm{min}), \mathrm{t}_{\text {major }}=21.331 \mathrm{~min}, \mathrm{t}_{\text {minor }}=33.696 \mathrm{~min}$; ee $=92 \% .{ }^{1} \mathrm{H}$ NMR $(400 \mathrm{MHz}$, $d_{6}$-DMSO) $\delta 8.51(\mathrm{~s}, 1 \mathrm{H}), 8.23(\mathrm{~d}, J=8.7 \mathrm{~Hz}, 2 \mathrm{H}), 7.72(\mathrm{~d}, J=8.7 \mathrm{~Hz}, 2 \mathrm{H}), 7.59(\mathrm{dd}, J=7.7,1.3$ $\mathrm{Hz}, 1 \mathrm{H}), 7.32(\mathrm{~s}, 1 \mathrm{H}), 7.28-7.20(\mathrm{~m}, 1 \mathrm{H}), 6.75(\mathrm{~d}, J=7.9 \mathrm{~Hz}, 1 \mathrm{H}), 6.67(\mathrm{t}, J=7.4 \mathrm{~Hz}, 1 \mathrm{H}), 5.89$ $(\mathrm{d}, J=2.2 \mathrm{~Hz}, 1 \mathrm{H})$. 

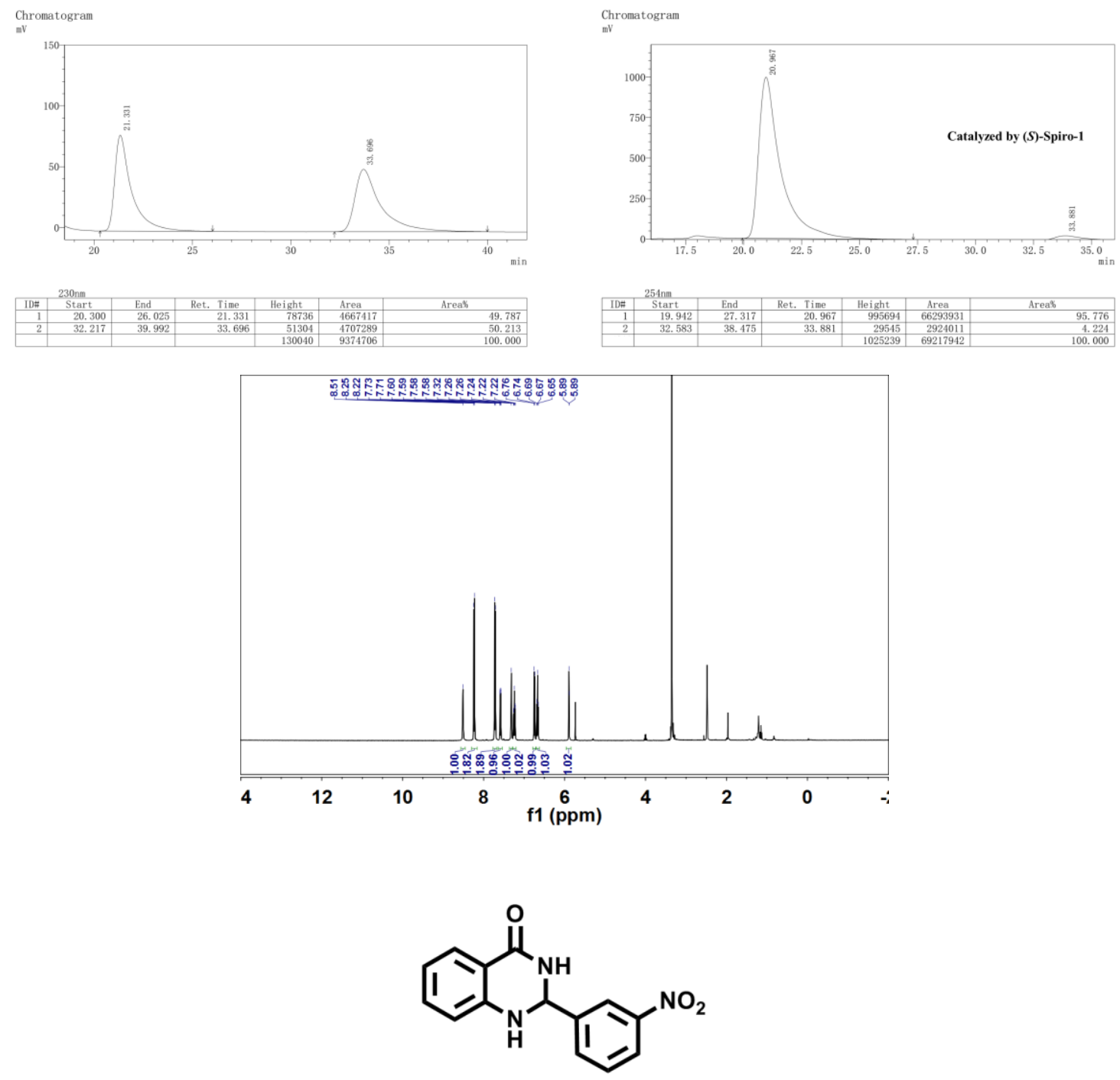

Enantiomeric excess was determined by HPLC with a chiralcel OD-H column (hexane/iPrOH $=$ $85 / 15,1.0 \mathrm{~mL} / \mathrm{min}), \mathrm{t}_{\text {major }}=31.233 \mathrm{~min}, \mathrm{t}_{\text {minor }}=56.677 \mathrm{~min}$; ee $=99 \% .{ }^{1} \mathrm{H} \mathrm{NMR}(400 \mathrm{MHz}$, $d_{6}$-DMSO) $\delta 8.51(\mathrm{~s}, 1 \mathrm{H}), 8.32(\mathrm{~s}, 1 \mathrm{H}), 8.23-8.11(\mathrm{~m}, 1 \mathrm{H}), 7.91(\mathrm{t}, J=7.8 \mathrm{~Hz}, 1 \mathrm{H}), 7.67(\mathrm{t}, J=$ $7.9 \mathrm{~Hz}, 1 \mathrm{H}), 7.58(\mathrm{~d}, J=7.7 \mathrm{~Hz}, 1 \mathrm{H}), 7.33(\mathrm{~s}, 1 \mathrm{H}), 7.29-7.19(\mathrm{~m}, 1 \mathrm{H}), 6.79-6.60(\mathrm{~m}, 2 \mathrm{H}), 5.91$ (s, 1H).
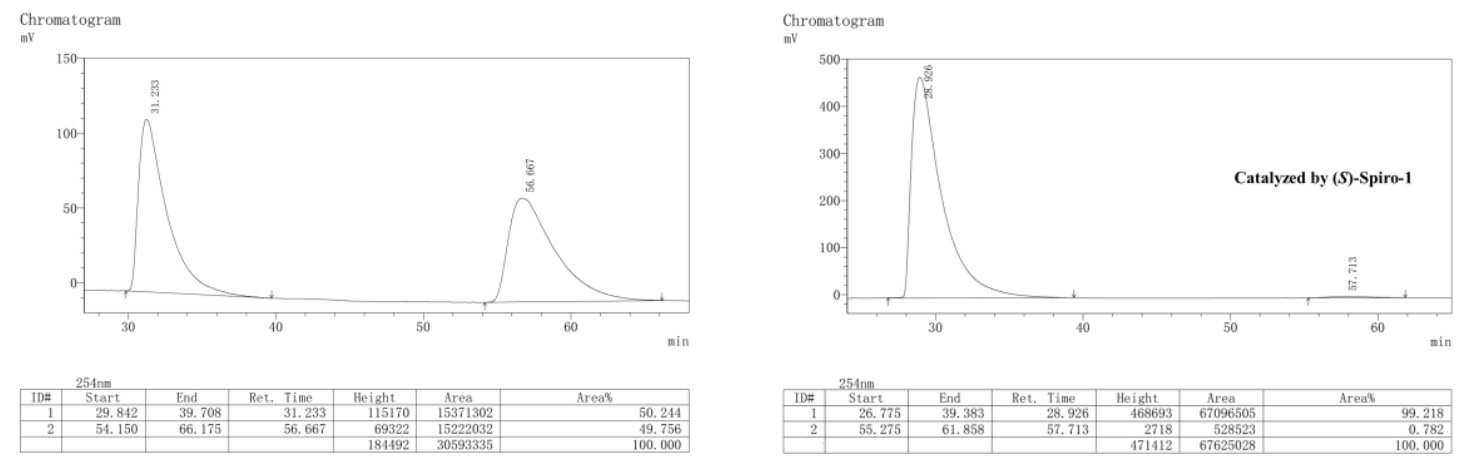

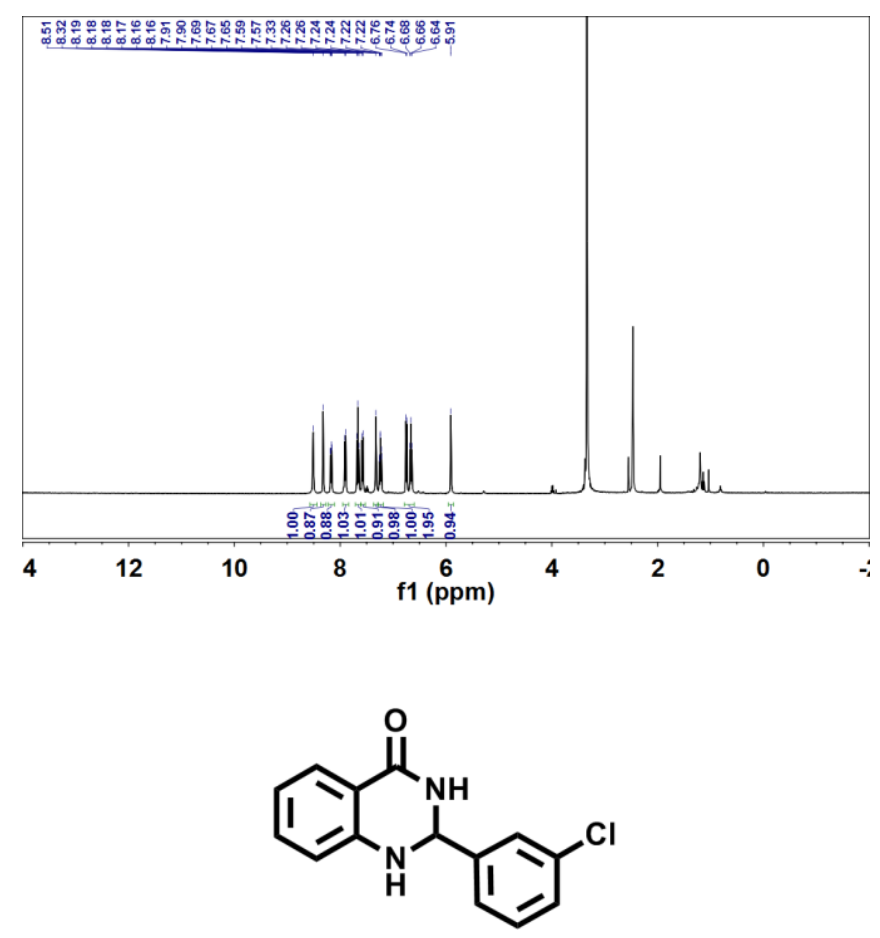

Enantiomeric excess was determined by HPLC with a chiralcel OD-H column (hexane/iPrOH $=$ $80 / 20,1.0 \mathrm{~mL} / \mathrm{min}), \mathrm{t}_{\text {major }}=12.553 \mathrm{~min}, \mathrm{t}_{\text {minor }}=15.963 \mathrm{~min}$; ee $=92 \% .{ }^{1} \mathrm{H} \mathrm{NMR}(400 \mathrm{MHz}$, $d_{6}$-DMSO) $\delta 8.39(\mathrm{~s}, 1 \mathrm{H}), 7.58(\mathrm{dd}, J=7.7,1.2 \mathrm{~Hz}, 1 \mathrm{H}), 7.50(\mathrm{~s}, 1 \mathrm{H}), 7.45-7.35(\mathrm{~m}, 3 \mathrm{H}), 7.29-$ $7.14(\mathrm{~m}, 2 \mathrm{H}), 6.73(\mathrm{~d}, J=7.7 \mathrm{~Hz}, 1 \mathrm{H}), 6.70-6.61(\mathrm{~m}, 1 \mathrm{H}), 5.76(\mathrm{~s}, 1 \mathrm{H})$.
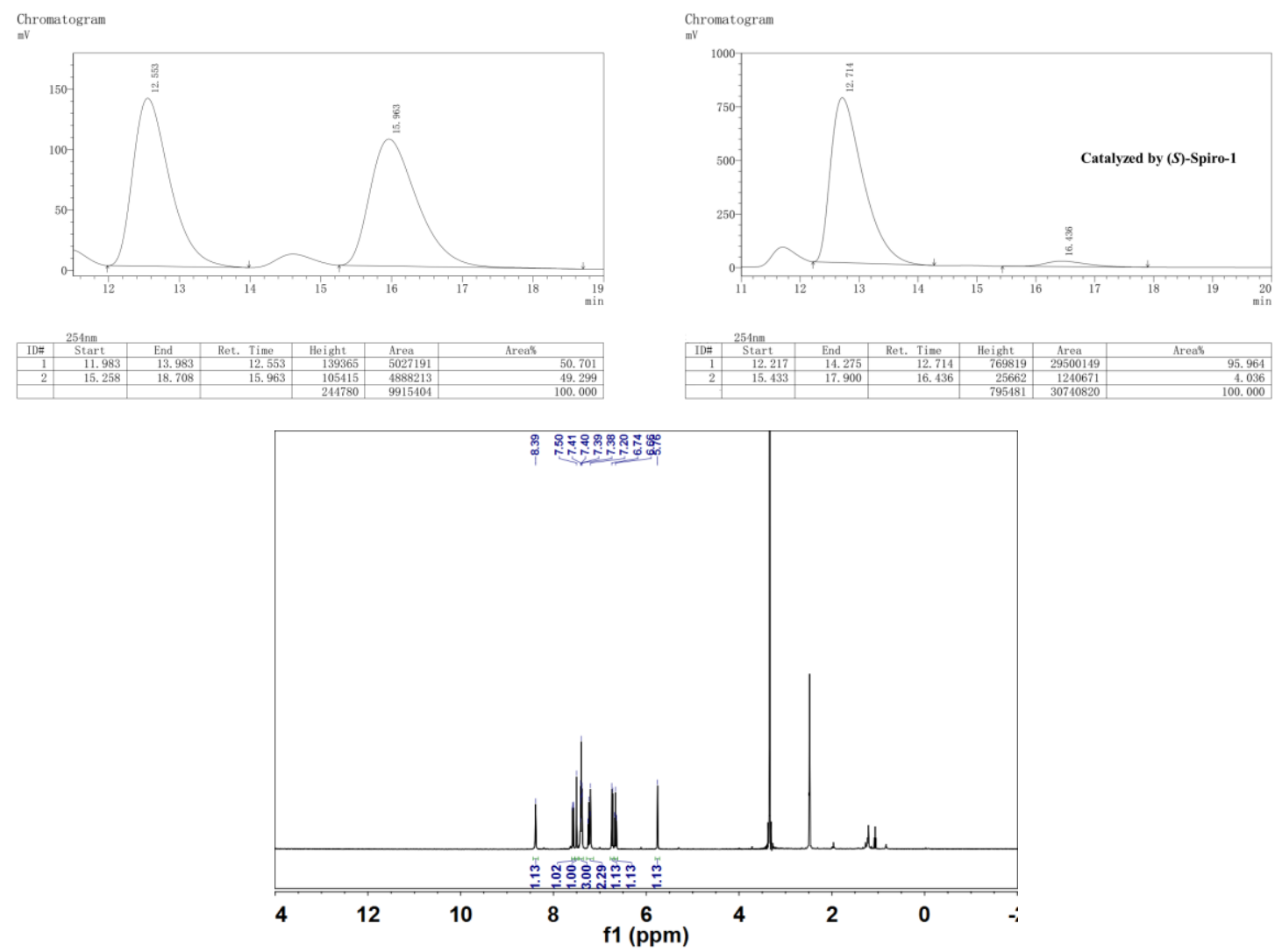


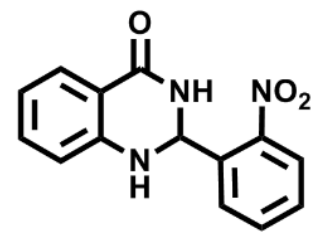

Enantiomeric excess was determined by HPLC with a chiralcel AD-H column (hexane/iPrOH $=$ $80 / 20,0.8 \mathrm{~mL} / \mathrm{min}), \mathrm{t}_{\text {major }}=26.834 \mathrm{~min}, \mathrm{t}_{\text {minor }}=29.361 \mathrm{~min}$; ee $=98 \% .{ }^{1} \mathrm{H} \mathrm{NMR}(400 \mathrm{MHz}$, $d_{6}$-DMSO) $\delta 8.21(\mathrm{~s}, 1 \mathrm{H}), 8.07-8.01(\mathrm{~m}, 1 \mathrm{H}), 7.87-7.73(\mathrm{~m}, 2 \mathrm{H}), 7.62(\mathrm{ddd}, J=13.9,6.7,1.3$ $\mathrm{Hz}, 2 \mathrm{H}), 7.28-7.19(\mathrm{~m}, 1 \mathrm{H}), 6.99$ (s, 1H), $6.80-6.64(\mathrm{~m}, 2 \mathrm{H}), 6.31$ (s, 1H).
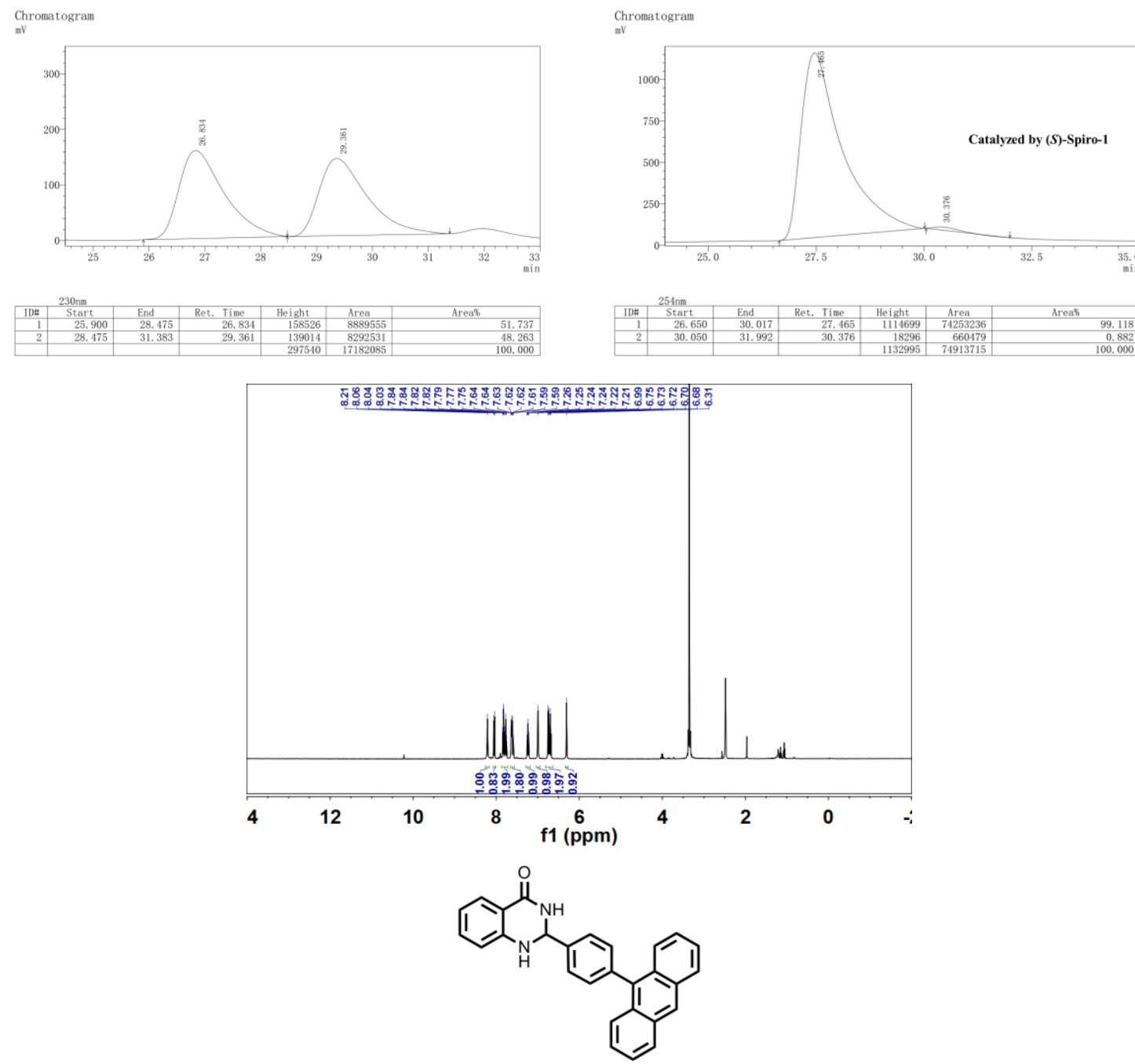

Enantiomeric excess was determined by HPLC with a chiralcel OD-H column (hexane/iPrOH = $80 / 20,1.2 \mathrm{~mL} / \mathrm{min}), \mathrm{t}_{\text {major }}=18.265 \mathrm{~min}, \mathrm{t}_{\text {minor }}=28.996 \mathrm{~min}$; ee $=91 \% .{ }^{1} \mathrm{H} \mathrm{NMR}(500 \mathrm{MHz}$, $d_{6}$-DMSO) $\delta 8.68(\mathrm{~s}, 1 \mathrm{H}), 8.46(\mathrm{~d}, J=14.8 \mathrm{~Hz}, 1 \mathrm{H}), 8.21-8.12(\mathrm{~m}, 2 \mathrm{H}), 7.76(\mathrm{~d}, J=8.1 \mathrm{~Hz}, 2 \mathrm{H}), 7.72$ $-7.66(\mathrm{~m}, 1 \mathrm{H}), 7.58-7.49(\mathrm{~m}, 4 \mathrm{H}), 7.48-7.39(\mathrm{~m}, 4 \mathrm{H}), 7.31(\mathrm{ddd}, J=8.2,5.3,1.6 \mathrm{~Hz}, 2 \mathrm{H}), 6.86(\mathrm{~d}$, $J=7.8 \mathrm{~Hz}, 1 \mathrm{H}), 6.77-6.69(\mathrm{~m}, 1 \mathrm{H}), 5.97(\mathrm{~s}, 1 \mathrm{H}) .{ }^{13} \mathrm{C}$ NMR (126 MHz, $d_{6}$-DMSO) $\delta 164.03,148.47$, $141.48,138.63,136.39,133.93,131.42,131.35,129.94,128.95,127.93,127.62,127.09,126.39$, $126.30,125.78,117.68,115.36,114.93,79.64,67.11$. 
Chromatogram

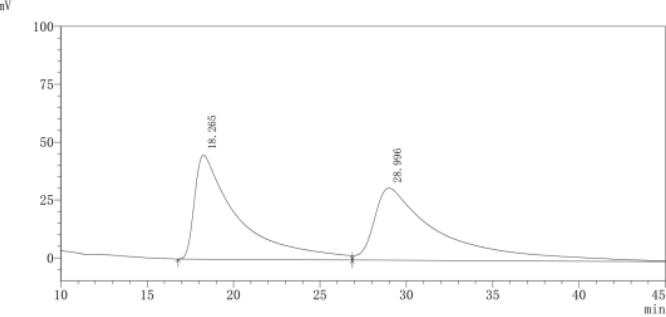

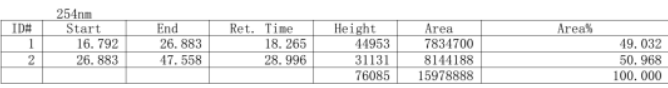

Chromatogram

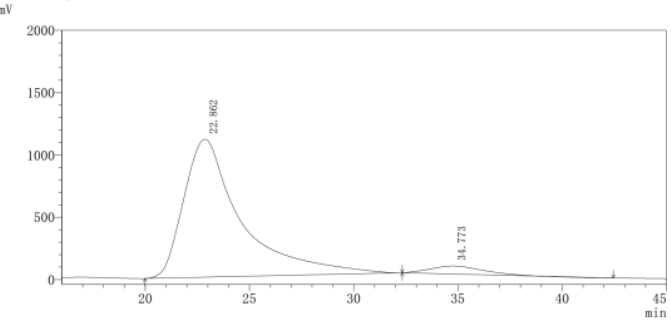

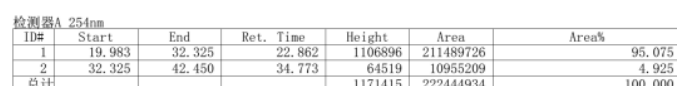

Chromatogram

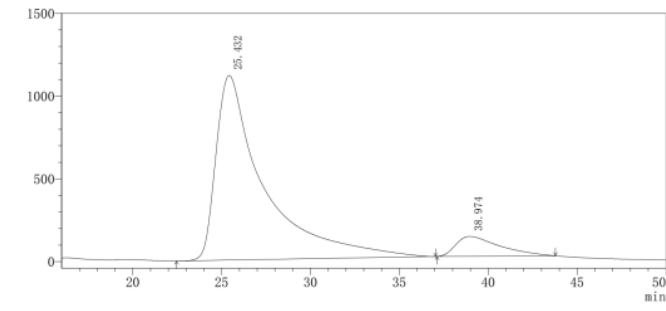

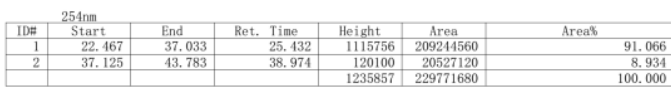

Chromatogram

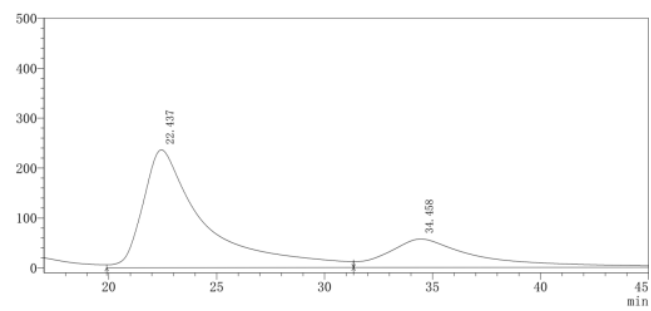

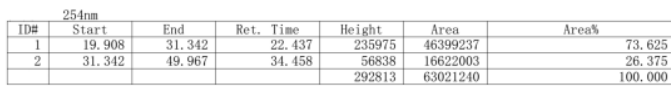

$\underset{\mathrm{mV}}{\text { Chromatogram }}$
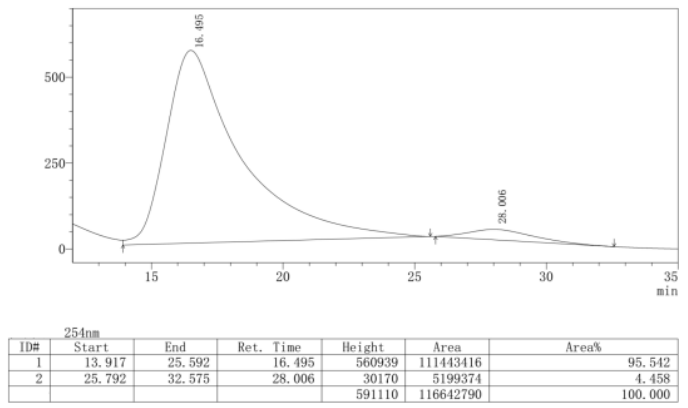

Chromatogram
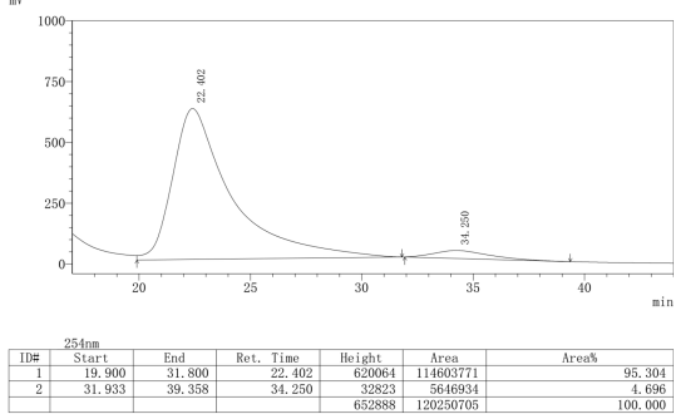

Chromatogran

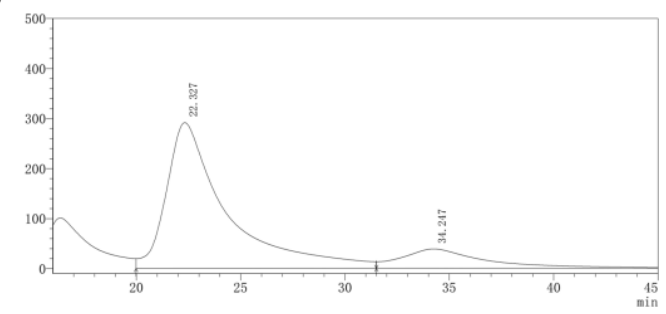

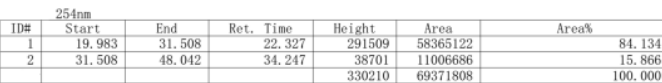



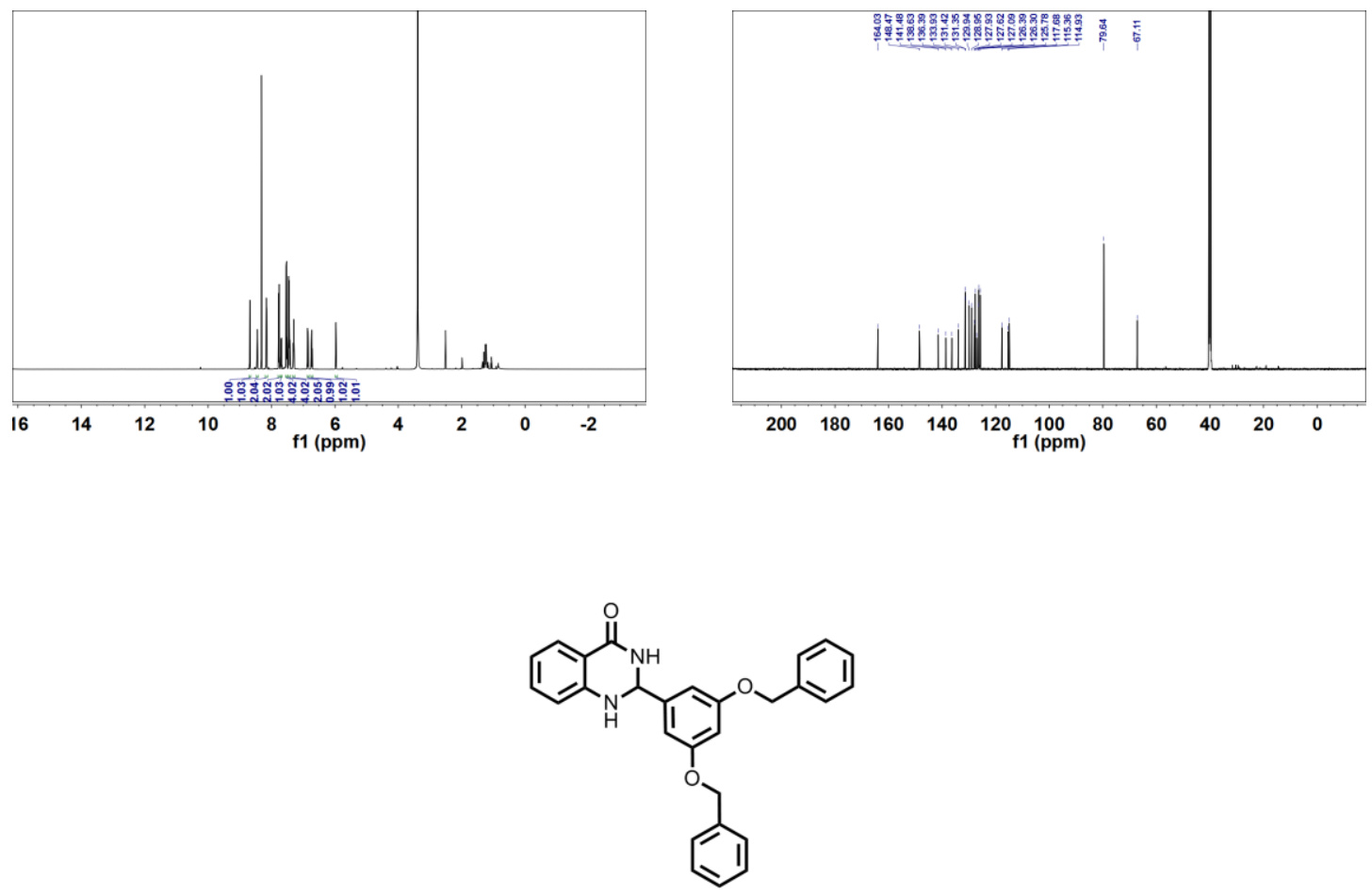

Enantiomeric excess was determined by HPLC with a chiralcel OD-H column (hexane/iPrOH = $60 / 40,1.2 \mathrm{~mL} / \mathrm{min}), \mathrm{t}_{\text {major }}=11.834 \mathrm{~min}, \mathrm{t}_{\text {minor }}=33.044 \mathrm{~min}$; ee $=77 \% .{ }^{1} \mathrm{H}$ NMR $(500 \mathrm{MHz}$, $d_{6}$-DMSO) $\delta 8.35(\mathrm{~s}, 1 \mathrm{H}), 7.65(\mathrm{dd}, J=7.7,1.4 \mathrm{~Hz}, 1 \mathrm{H}), 7.48-7.30(\mathrm{~m}, 10 \mathrm{H}), 7.30-7.21(\mathrm{~m}, 1 \mathrm{H})$, $7.21-7.12(\mathrm{~m}, 1 \mathrm{H}), 6.84-6.75(\mathrm{~m}, 3 \mathrm{H}), 6.69(\mathrm{dt}, J=4.2,1.4 \mathrm{~Hz}, 2 \mathrm{H}), 5.71(\mathrm{~s}, 1 \mathrm{H}), 5.08(\mathrm{~s}, 4 \mathrm{H}) .{ }^{13} \mathrm{C}$ NMR $\left(126 \mathrm{MHz}, d_{6}\right.$-DMSO) $\delta 164.00,159.93,148.18,144.85,137.30,133.84,128.93,128.38,128.26$, $127.85,117.62,115.43,114.92,106.49,101.74,69.87,66.61$.
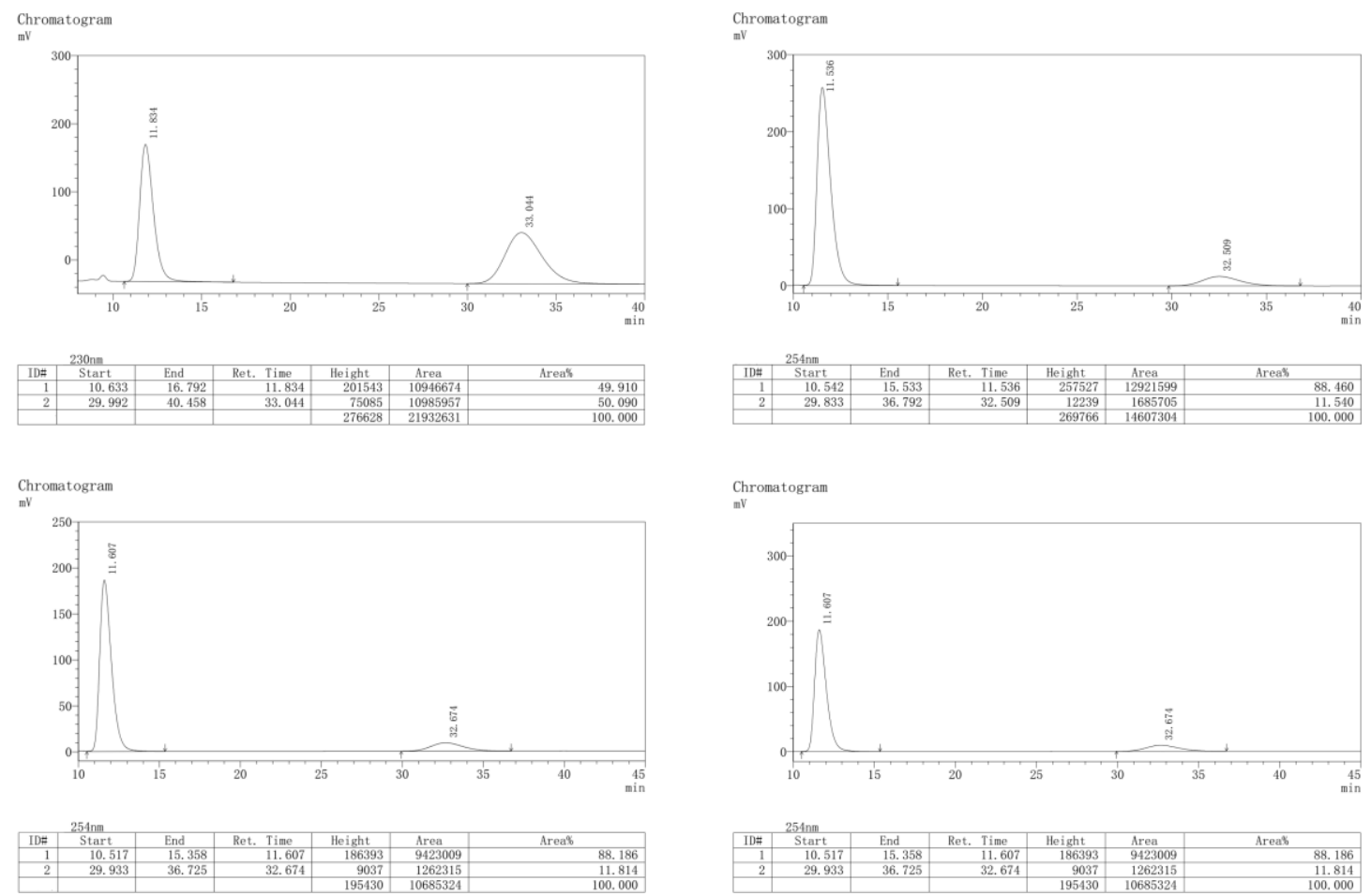

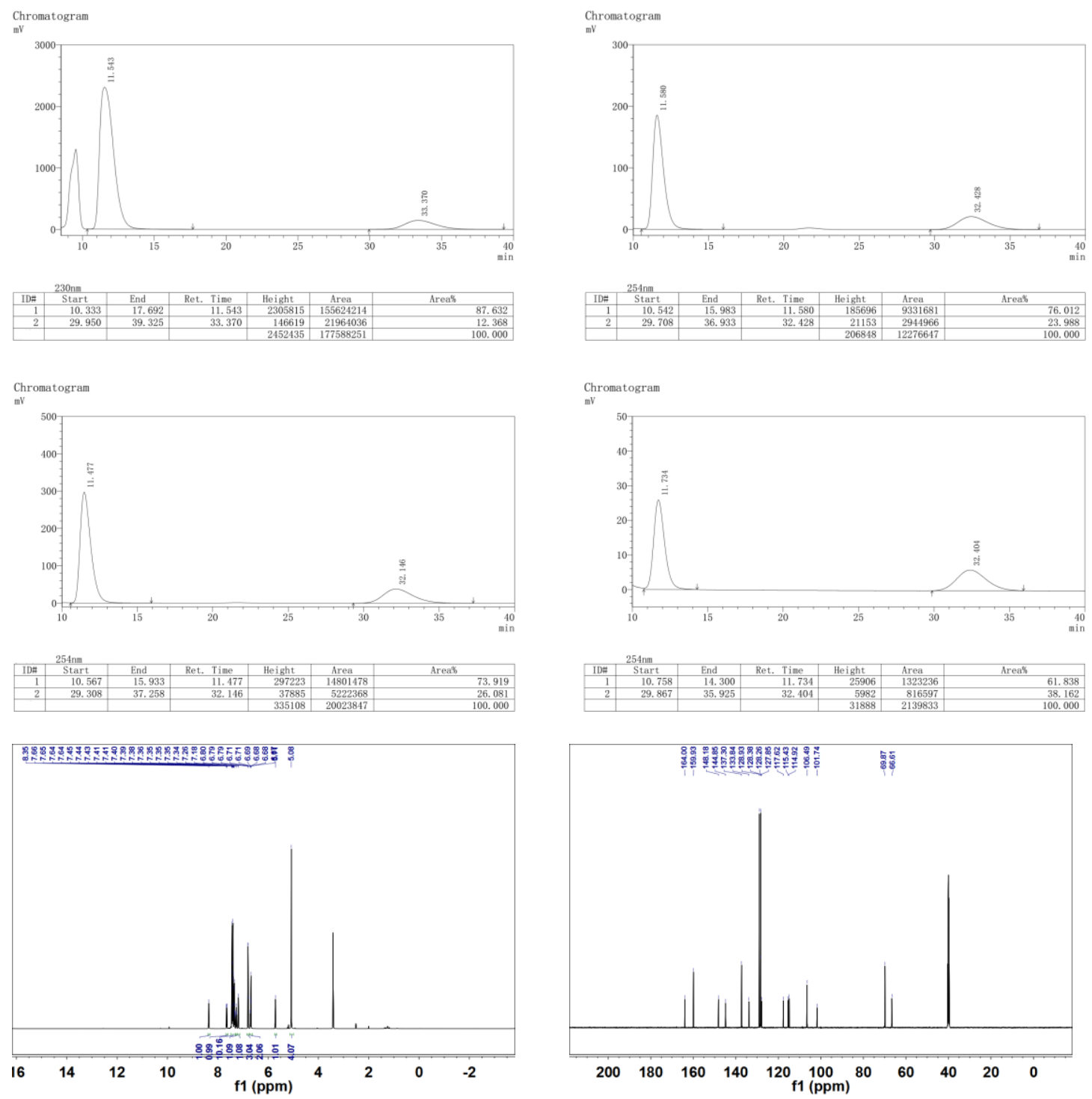

20.2 Tandem deacetalization/acetalization reaction of 2 -aminobenzamide with arylaldehydes dimethyl acetal
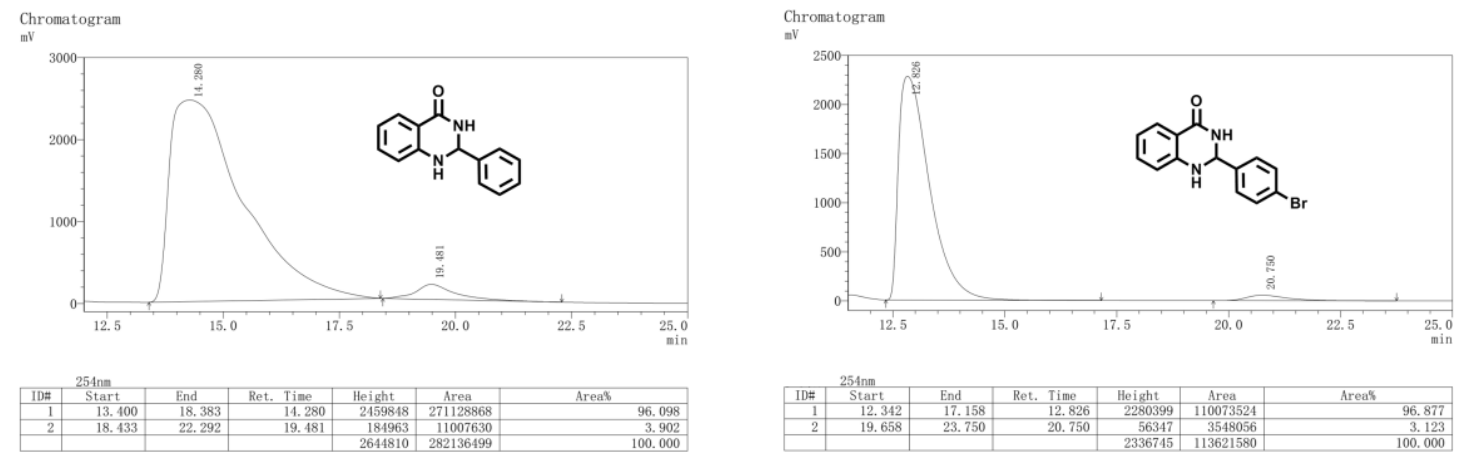

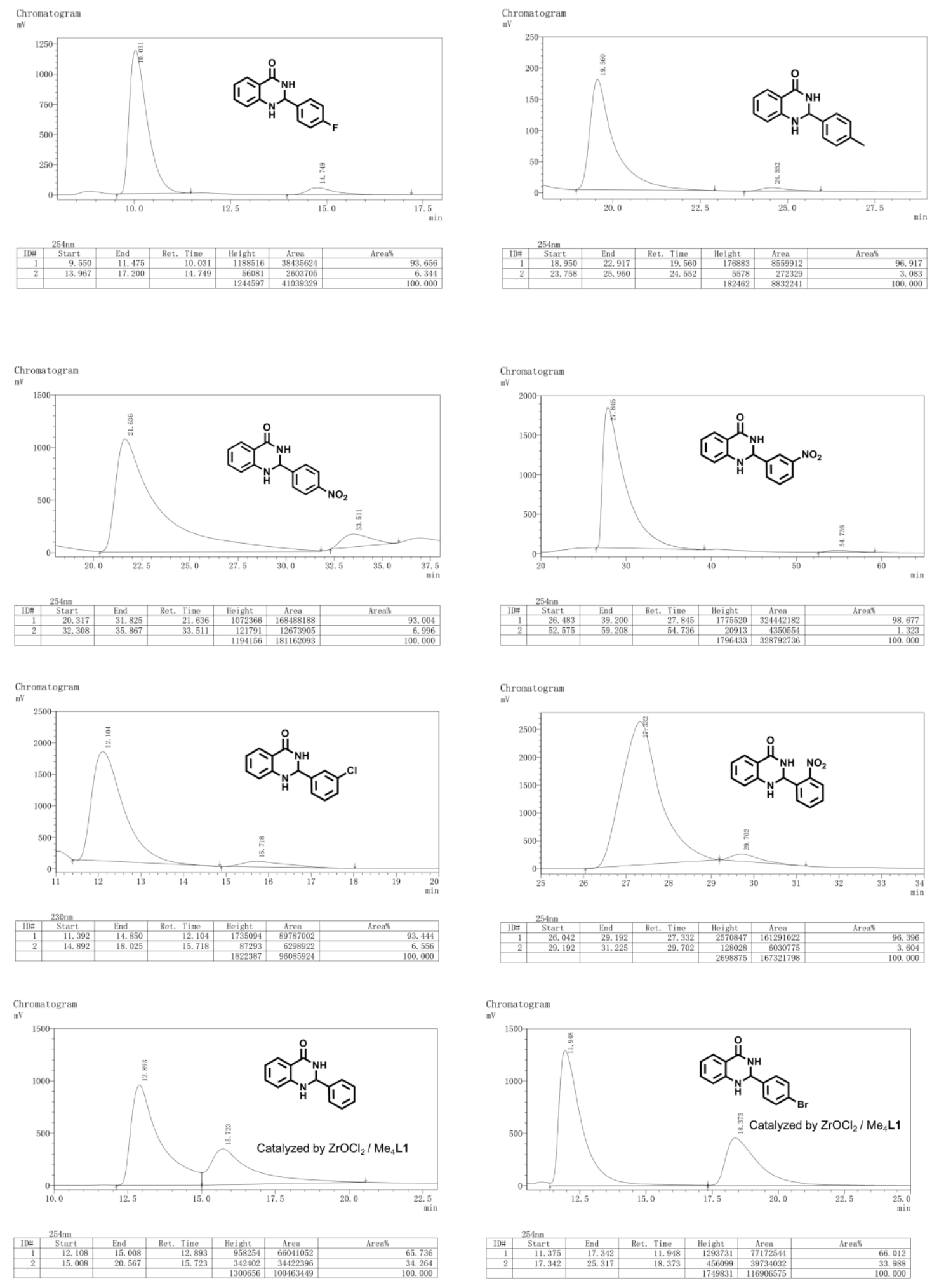

S48 
Chromatogram
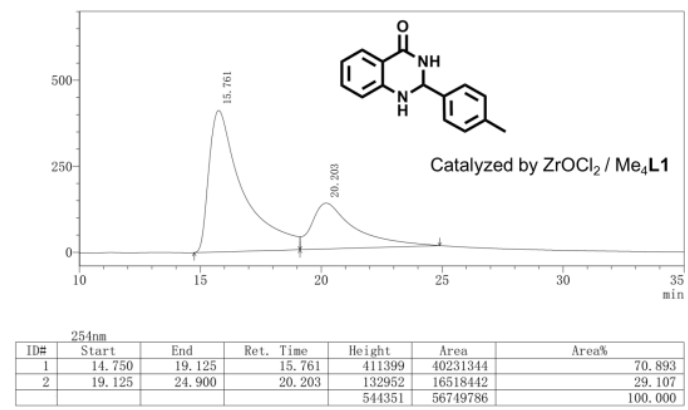

Chromatogram
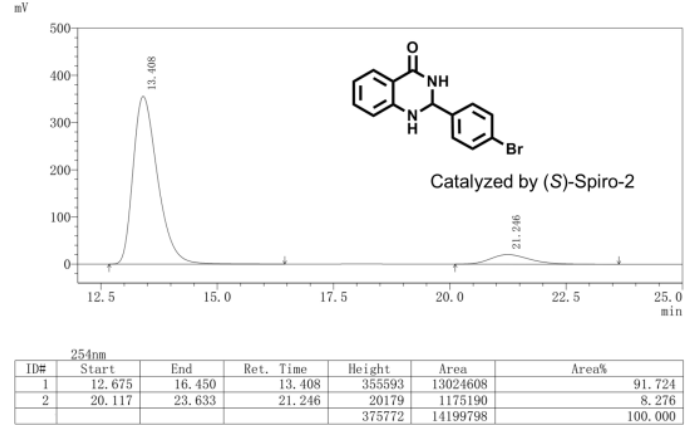

Chromatogram
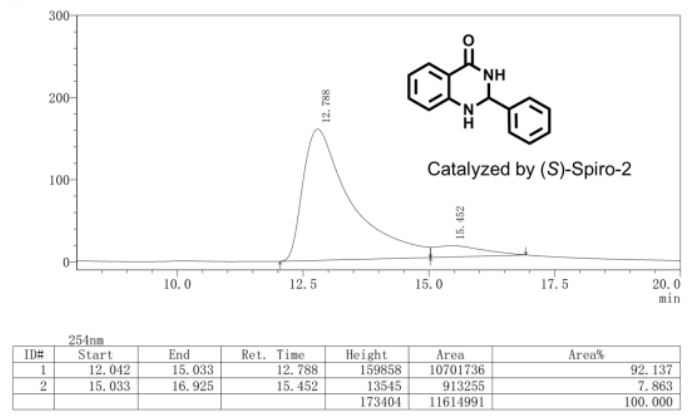

Chromatogran
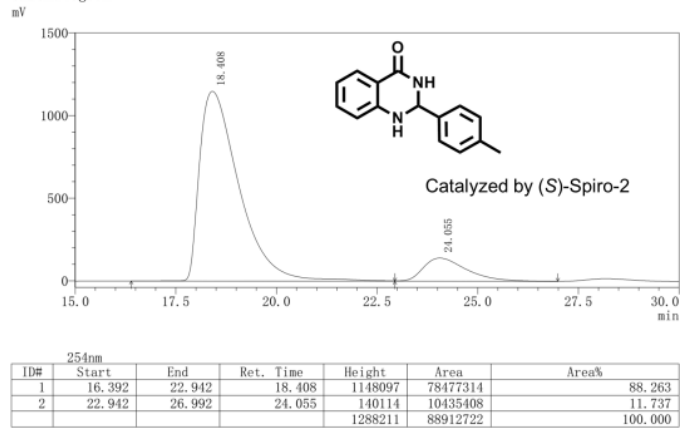

20.3 Tandem deacetalization/acetalization reaction of 2 -aminobenzamide with arylaldehydes cyclic acetal
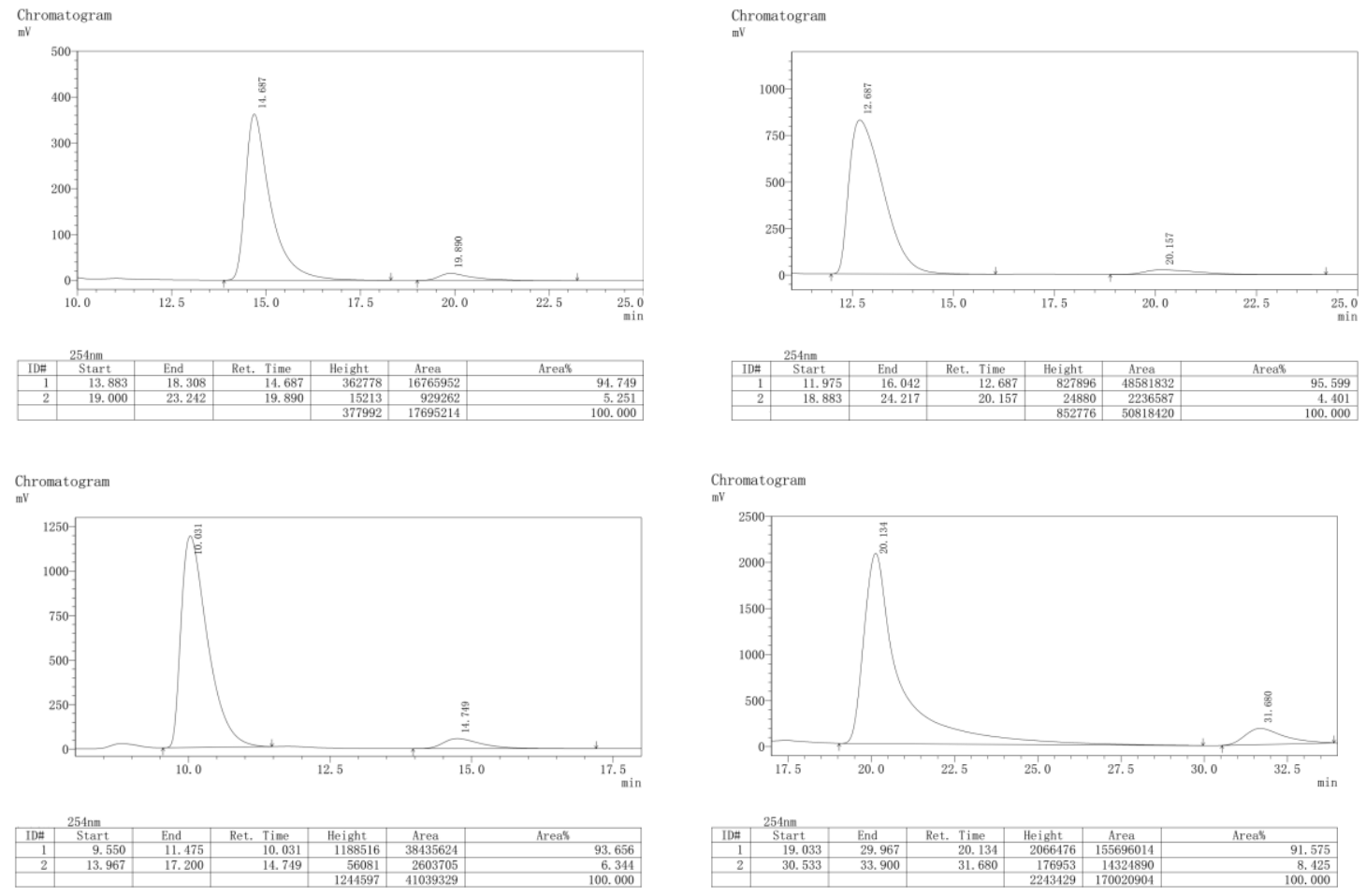
Chromatogram
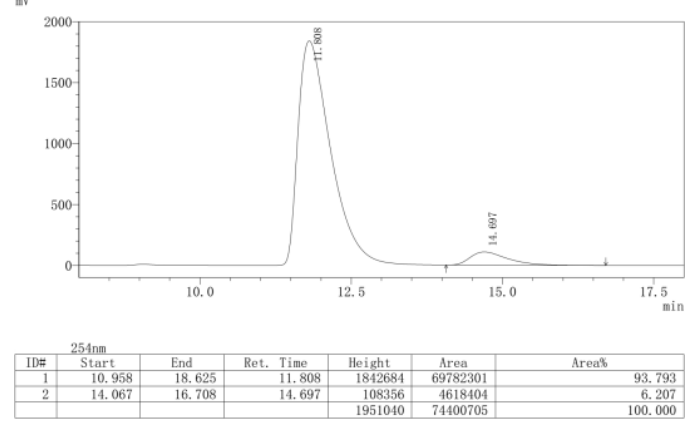

Chromatogram
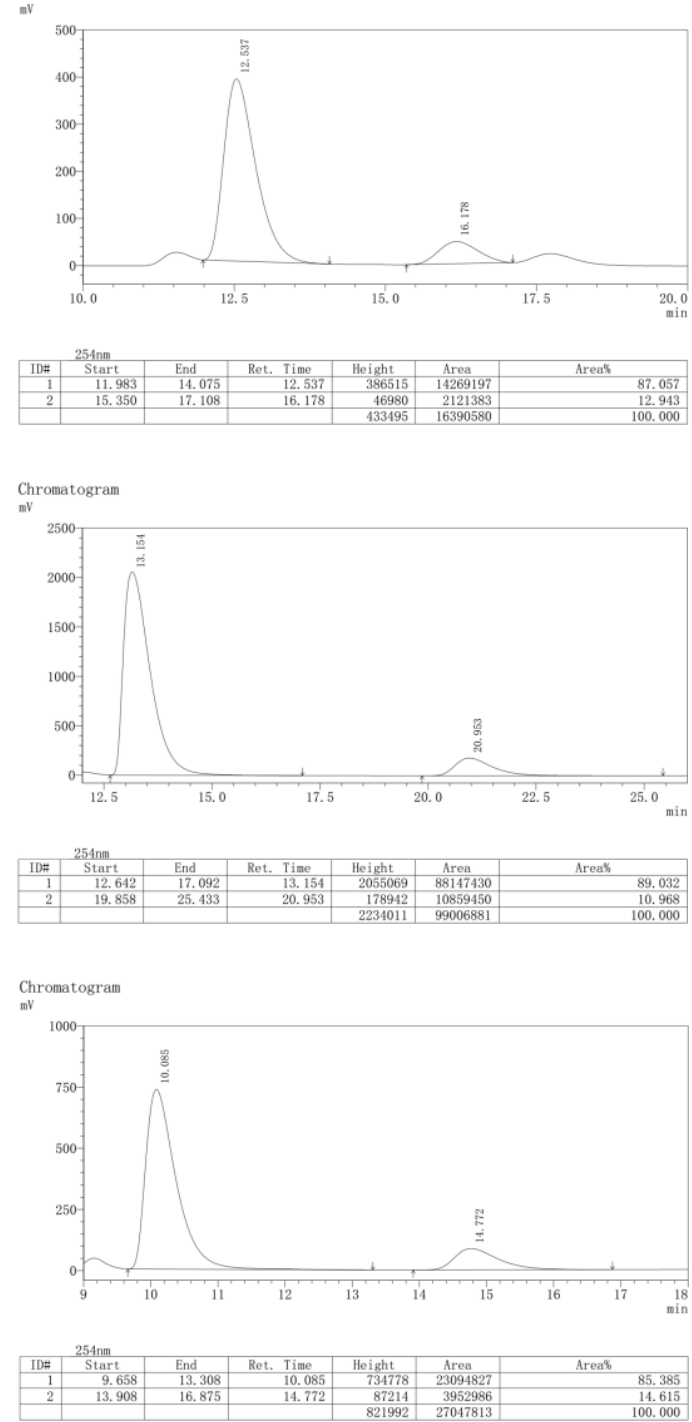
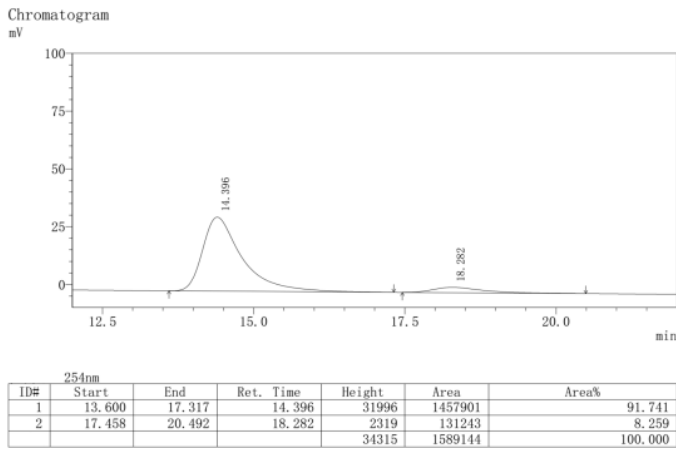

Chromatogran
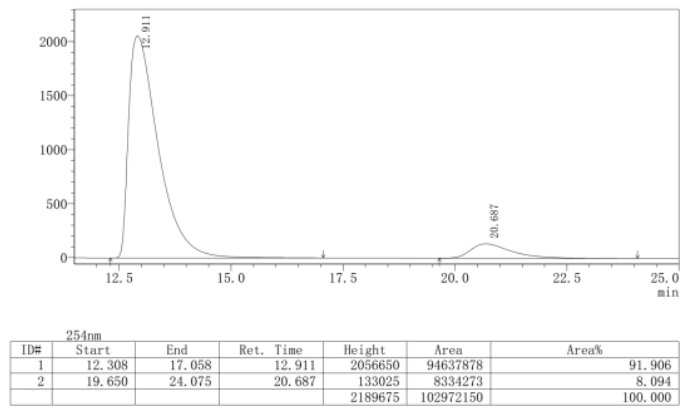

Chromatogram
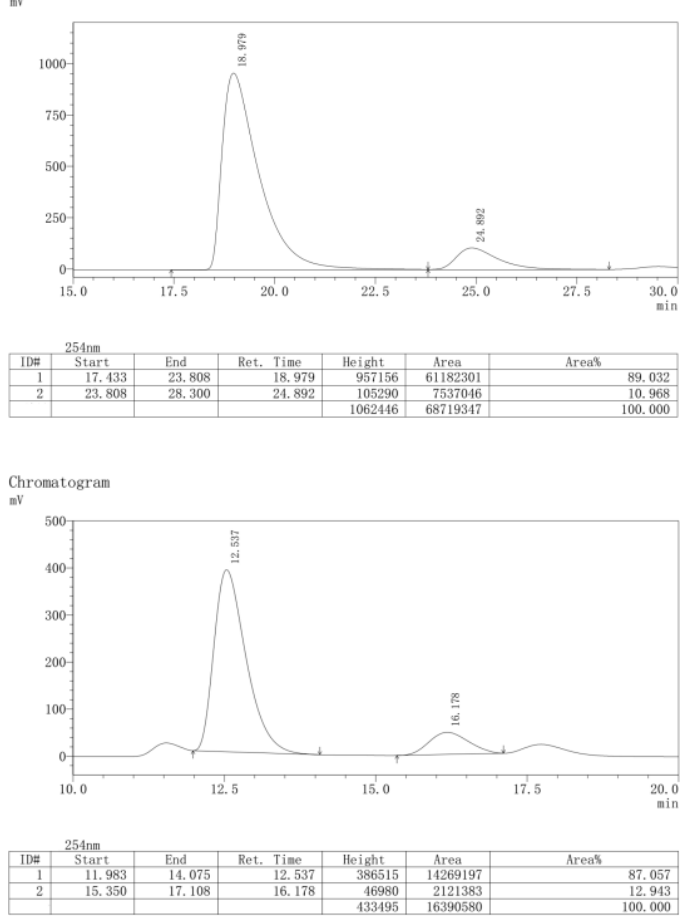

20.4 Friedel-Crafts alkylation of indole with $\mathrm{N}$-Sulfonyl aryl aldimines

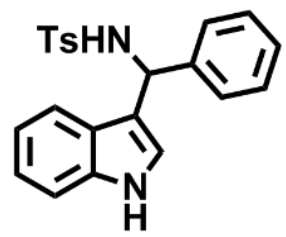

Enantiomeric excess was determined by HPLC with a chiralcel OD-H column (hexane/iPrOH = 
$70 / 30,0.6 \mathrm{~mL} / \mathrm{min}), \mathrm{t}_{\text {minor }}=15.458 \mathrm{~min}, \mathrm{t}_{\text {major }}=24.670 \mathrm{~min}$; ee $=99 \% .{ }^{1} \mathrm{H} \mathrm{NMR}(400 \mathrm{MHz}$, $d_{6}$-DMSO) $\delta 10.85(\mathrm{~s}, 1 \mathrm{H}), 8.47(\mathrm{~d}, J=8.9 \mathrm{~Hz}, 1 \mathrm{H}), 7.47(\mathrm{~d}, J=7.9 \mathrm{~Hz}, 2 \mathrm{H}), 7.32-7.21(\mathrm{~m}, 4 \mathrm{H})$, $7.19-7.07(\mathrm{~m}, 4 \mathrm{H}), 7.01(\mathrm{dd}, J=8.0,7.2 \mathrm{~Hz}, 1 \mathrm{H}), 6.86(\mathrm{dd}, J=7.9,7.1 \mathrm{~Hz}, 1 \mathrm{H}), 6.75(\mathrm{~d}, J=2.4$ $\mathrm{Hz}, 1 \mathrm{H}), 5.72(\mathrm{~d}, J=8.8 \mathrm{~Hz}, 1 \mathrm{H}), 2.26(\mathrm{~s}, 3 \mathrm{H})$.
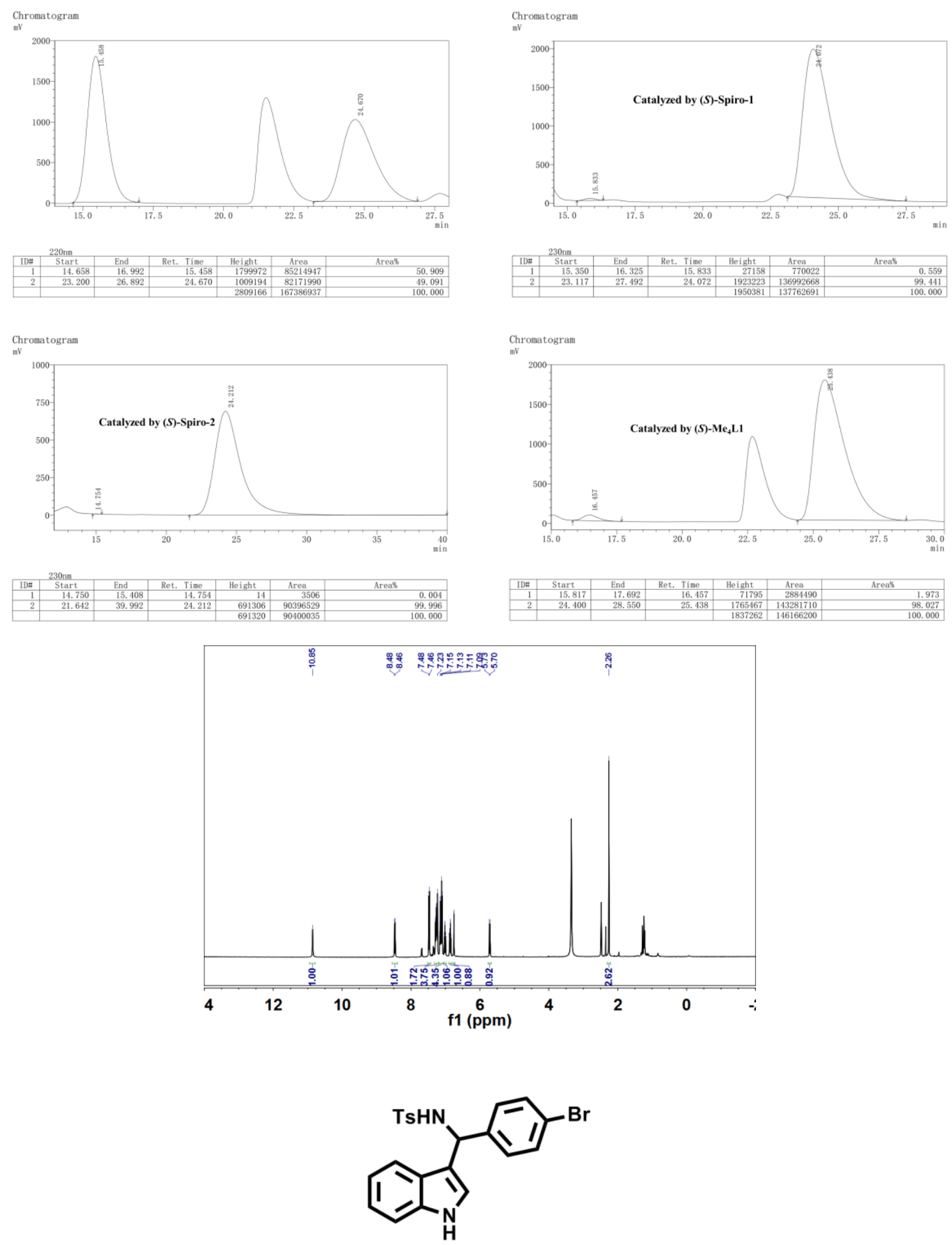

Enantiomeric excess was determined by HPLC with a chiralcel OD-H column (hexane/iPrOH = $70 / 30,1.0 \mathrm{~mL} / \mathrm{min}), \mathrm{t}_{\text {minor }}=10.907 \mathrm{~min}, \mathrm{t}_{\text {major }}=17.014 \mathrm{~min}$; ee $=99.9 \% .{ }^{1} \mathrm{H} \mathrm{NMR}(400 \mathrm{MHz}$, $d_{6}$-DMSO) $\delta 11.01(\mathrm{~s}, 1 \mathrm{H}), 8.68(\mathrm{~d}, J=8.6 \mathrm{~Hz}, 1 \mathrm{H}), 8.05(\mathrm{t}, J=9.6 \mathrm{~Hz}, 2 \mathrm{H}), 7.53(\mathrm{dd}, J=19.0$, $8.4 \mathrm{~Hz}, 4 \mathrm{H}), 7.32(\mathrm{~d}, J=8.4 \mathrm{~Hz}, 2 \mathrm{H}), 7.15(\mathrm{~d}, J=8.1 \mathrm{~Hz}, 2 \mathrm{H}), 7.07(\mathrm{t}, J=7.7 \mathrm{~Hz}, 1 \mathrm{H}), 6.92(\mathrm{t}, J$ 
$=7.6 \mathrm{~Hz}, 1 \mathrm{H}), 6.79(\mathrm{~d}, J=2.2 \mathrm{~Hz}, 1 \mathrm{H}), 5.88(\mathrm{~d}, J=8.6 \mathrm{~Hz}, 1 \mathrm{H}), 2.27(\mathrm{~s}, 3 \mathrm{H})$.
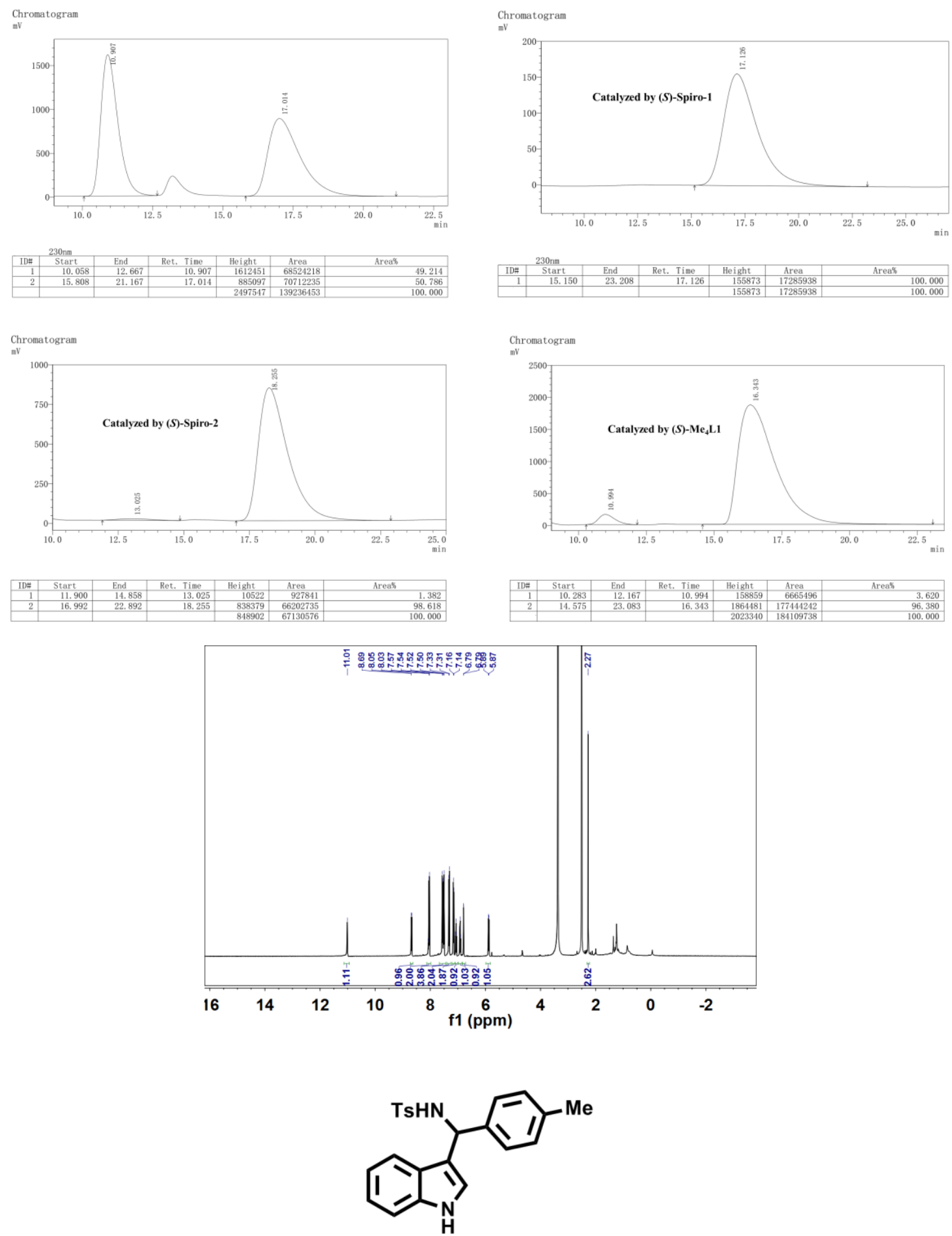

Enantiomeric excess was determined by HPLC with a chiralcel OD-H column (hexane/iPrOH = $70 / 30,0.6 \mathrm{~mL} / \mathrm{min}), \mathrm{t}_{\text {minor }}=13.957 \mathrm{~min}, \mathrm{t}_{\text {major }}=23.489 \mathrm{~min}$; ee $=96 \% .{ }^{1} \mathrm{H} \mathrm{NMR}(400 \mathrm{MHz}$, $d_{6}$-DMSO) $\delta 10.80(\mathrm{~d}, J=26.1 \mathrm{~Hz}, 1 \mathrm{H}), 8.40(\mathrm{~d}, J=8.8 \mathrm{~Hz}, 1 \mathrm{H}), 7.49-7.44(\mathrm{~m}, 2 \mathrm{H}), 7.26(\mathrm{dd}, J$ $=6.8,5.8 \mathrm{~Hz}, 2 \mathrm{H}), 7.09(\mathrm{dd}, J=15.2,8.7 \mathrm{~Hz}, 4 \mathrm{H}), 7.01(\mathrm{t}, J=7.5 \mathrm{~Hz}, 1 \mathrm{H}), 6.94(\mathrm{~d}, J=7.3 \mathrm{~Hz}$, $2 \mathrm{H}), 6.85(\mathrm{t}, J=7.6 \mathrm{~Hz}, 1 \mathrm{H}), 6.77(\mathrm{~s}, 1 \mathrm{H}), 5.66(\mathrm{~d}, J=8.7 \mathrm{~Hz}, 1 \mathrm{H}), 2.26(\mathrm{~s}, 3 \mathrm{H}), 2.19(\mathrm{~s}, 3 \mathrm{H})$. 

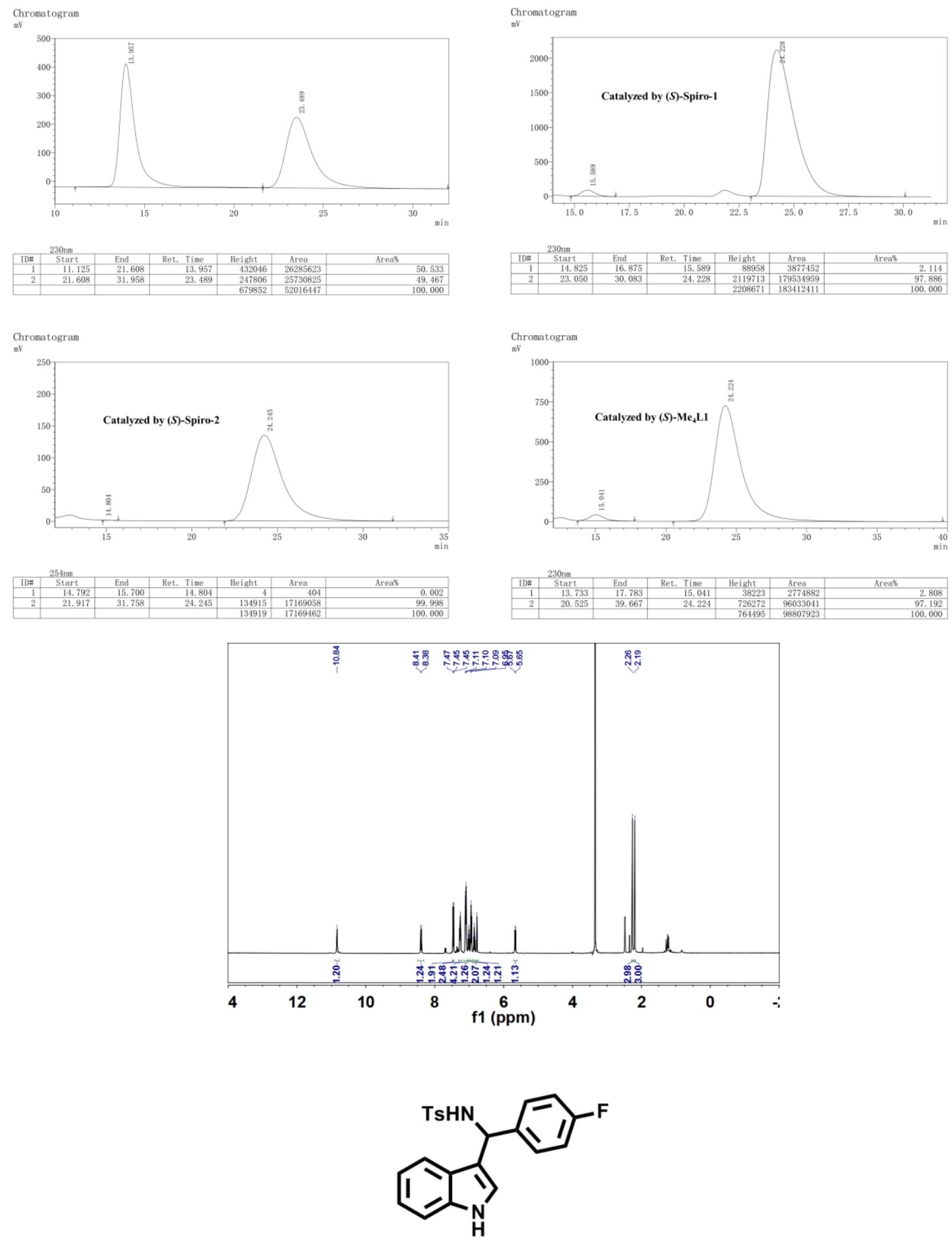

Enantiomeric excess was determined by HPLC with a chiralcel OD-H column (hexane/iPrOH = $70 / 30,0.6 \mathrm{~mL} / \mathrm{min}), \mathrm{t}_{\text {minor }}=17.766 \mathrm{~min}, \mathrm{t}_{\text {major }}=26.204 \mathrm{~min}$; ee $=99.9 \% .{ }^{1} \mathrm{H} \mathrm{NMR}(400 \mathrm{MHz}$, $d_{6}$-DMSO) $\delta 10.97(\mathrm{~s}, 1 \mathrm{H}), 8.64(\mathrm{~d}, J=8.6 \mathrm{~Hz}, 1 \mathrm{H}), 8.09-7.89(\mathrm{~m}, 2 \mathrm{H}), 7.52(\mathrm{ddd}, J=18.0,6.2$, $2.1 \mathrm{~Hz}, 4 \mathrm{H}$ ), 7.29 (dd, $J=8.2,0.7 \mathrm{~Hz}, 2 \mathrm{H}), 7.17-7.09$ (m, 2H), 7.04 (ddd, $J=8.3,7.1,1.0 \mathrm{~Hz}$, $1 \mathrm{H}), 6.89(\mathrm{ddd}, J=7.9,7.1,1.0 \mathrm{~Hz}, 1 \mathrm{H}), 6.77(\mathrm{~d}, J=2.5 \mathrm{~Hz}, 1 \mathrm{H}), 5.86(\mathrm{~d}, J=8.6 \mathrm{~Hz}, 1 \mathrm{H}), 2.25(\mathrm{~s}$, $3 \mathrm{H})$. 

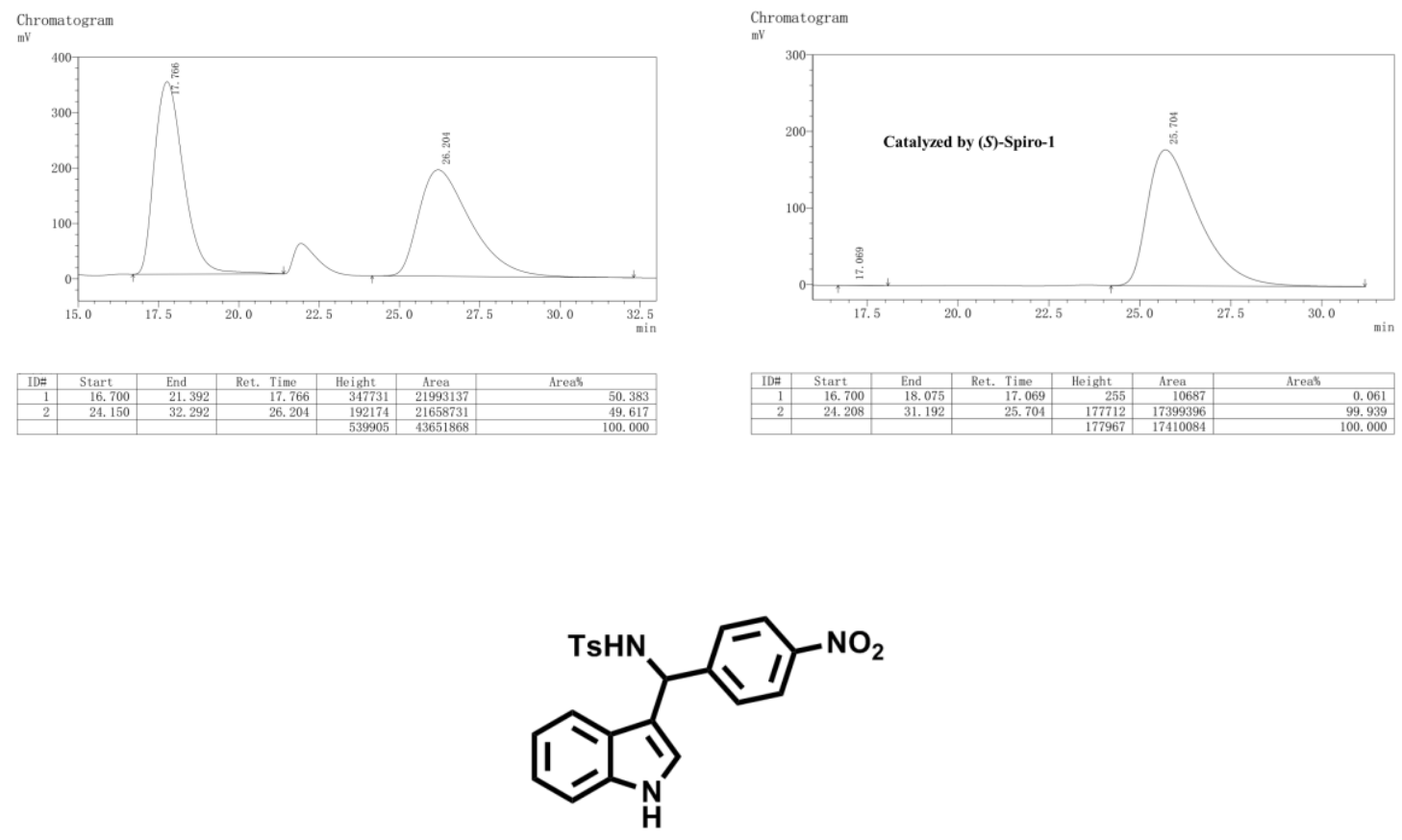

Enantiomeric excess was determined by HPLC with a chiralcel OD-H column (hexane/iPrOH $=$ $60 / 40,1.0 \mathrm{~mL} / \mathrm{min}), \mathrm{t}_{\text {minor }}=10.815 \mathrm{~min}, \mathrm{t}_{\text {major }}=17.189 \mathrm{~min}$; ee $=95 \% .{ }^{1} \mathrm{H} \mathrm{NMR}(400 \mathrm{MHz}$, $d_{6}$-DMSO) $\delta 10.97(\mathrm{~s}, 1 \mathrm{H}), 8.64(\mathrm{~d}, J=8.6 \mathrm{~Hz}, 1 \mathrm{H}), 8.06-7.96(\mathrm{~m}, 2 \mathrm{H}), 7.52$ (ddd, $J=18.0,6.2$, $2.1 \mathrm{~Hz}, 4 \mathrm{H}), 7.32-7.25(\mathrm{~m}, 2 \mathrm{H}), 7.17-7.07(\mathrm{~m}, 2 \mathrm{H}), 7.04$ (ddd, $J=8.3,7.1,1.0 \mathrm{~Hz}, 1 \mathrm{H}), 6.89$ (ddd, $J=7.9,7.1,1.0 \mathrm{~Hz}, 1 \mathrm{H}), 6.77(\mathrm{~d}, J=2.5 \mathrm{~Hz}, 1 \mathrm{H}), 5.86(\mathrm{~d}, J=8.6 \mathrm{~Hz}, 1 \mathrm{H}), 2.25(\mathrm{~s}, 3 \mathrm{H})$.
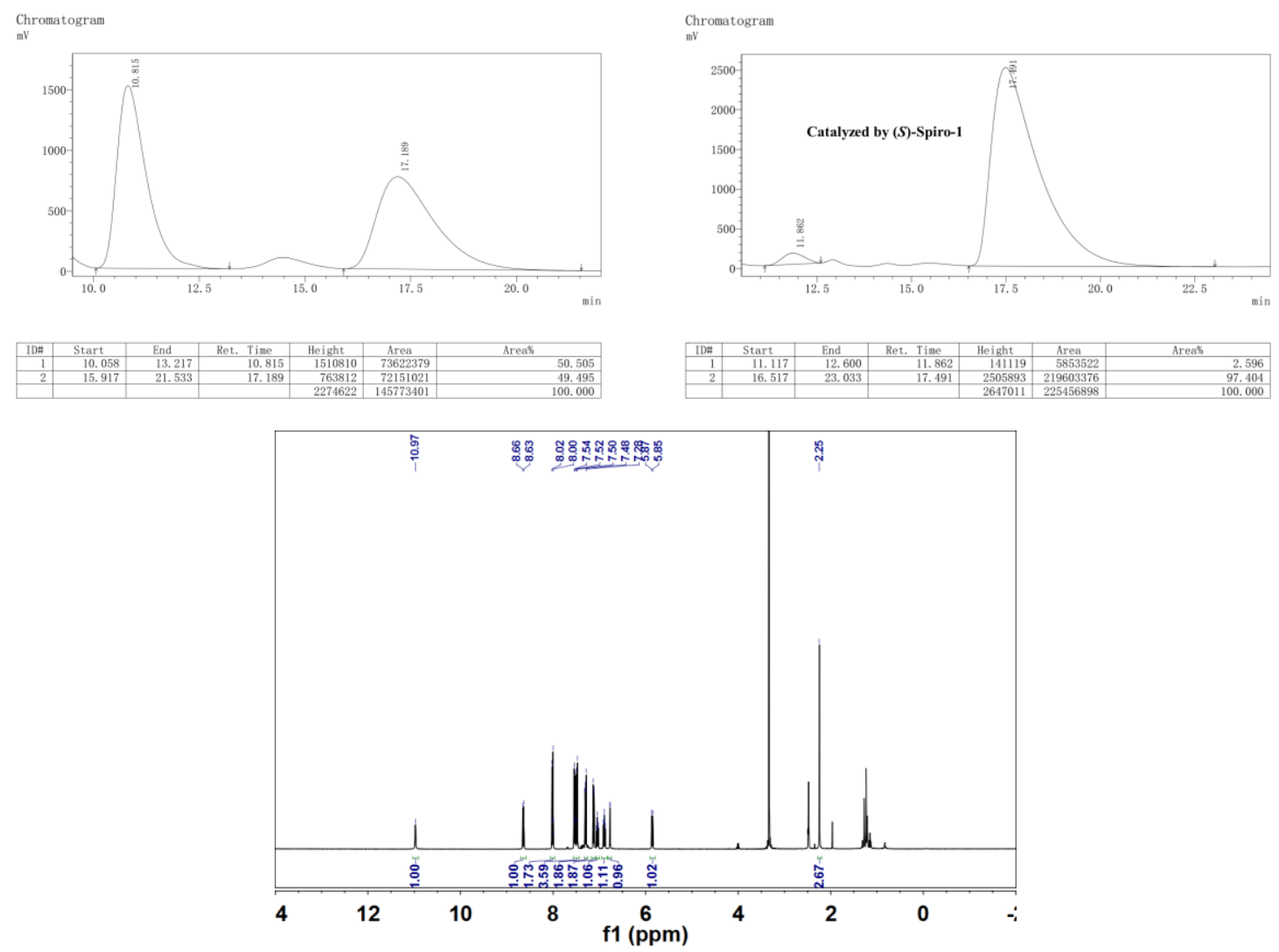


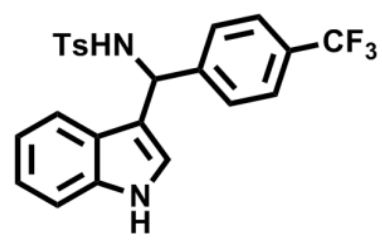

Enantiomeric excess was determined by HPLC with a chiralcel OD-H column (hexane/iPrOH = $70 / 30,0.6 \mathrm{~mL} / \mathrm{min}), \mathrm{t}_{\text {minor }}=17.215 \mathrm{~min}, \mathrm{t}_{\text {major }}=26.273 \mathrm{~min}$; ee $=99.9 \% .{ }^{1} \mathrm{H} \mathrm{NMR}(400 \mathrm{MHz}$, $d_{6}$-DMSO) $\delta 10.92(\mathrm{~s}, 1 \mathrm{H}), 8.57(\mathrm{~d}, J=8.9 \mathrm{~Hz}, 1 \mathrm{H}), 7.68(\mathrm{~d}, J=8.2 \mathrm{~Hz}, 2 \mathrm{H}), 7.45(\mathrm{~s}, 4 \mathrm{H}), 7.34(\mathrm{~d}$, $J=7.3 \mathrm{~Hz}, 3 \mathrm{H}), 7.30(\mathrm{~d}, J=8.1 \mathrm{~Hz}, 1 \mathrm{H}), 7.25(\mathrm{~s}, 2 \mathrm{H}), 7.03(\mathrm{~d}, J=8.0 \mathrm{~Hz}, 1 \mathrm{H}), 6.90(\mathrm{t}, J=7.5 \mathrm{~Hz}$, $1 \mathrm{H}), 6.75(\mathrm{~d}, J=2.3 \mathrm{~Hz}, 1 \mathrm{H}), 5.80(\mathrm{~d}, J=8.9 \mathrm{~Hz}, 1 \mathrm{H}), 2.35$ (s, 3H).
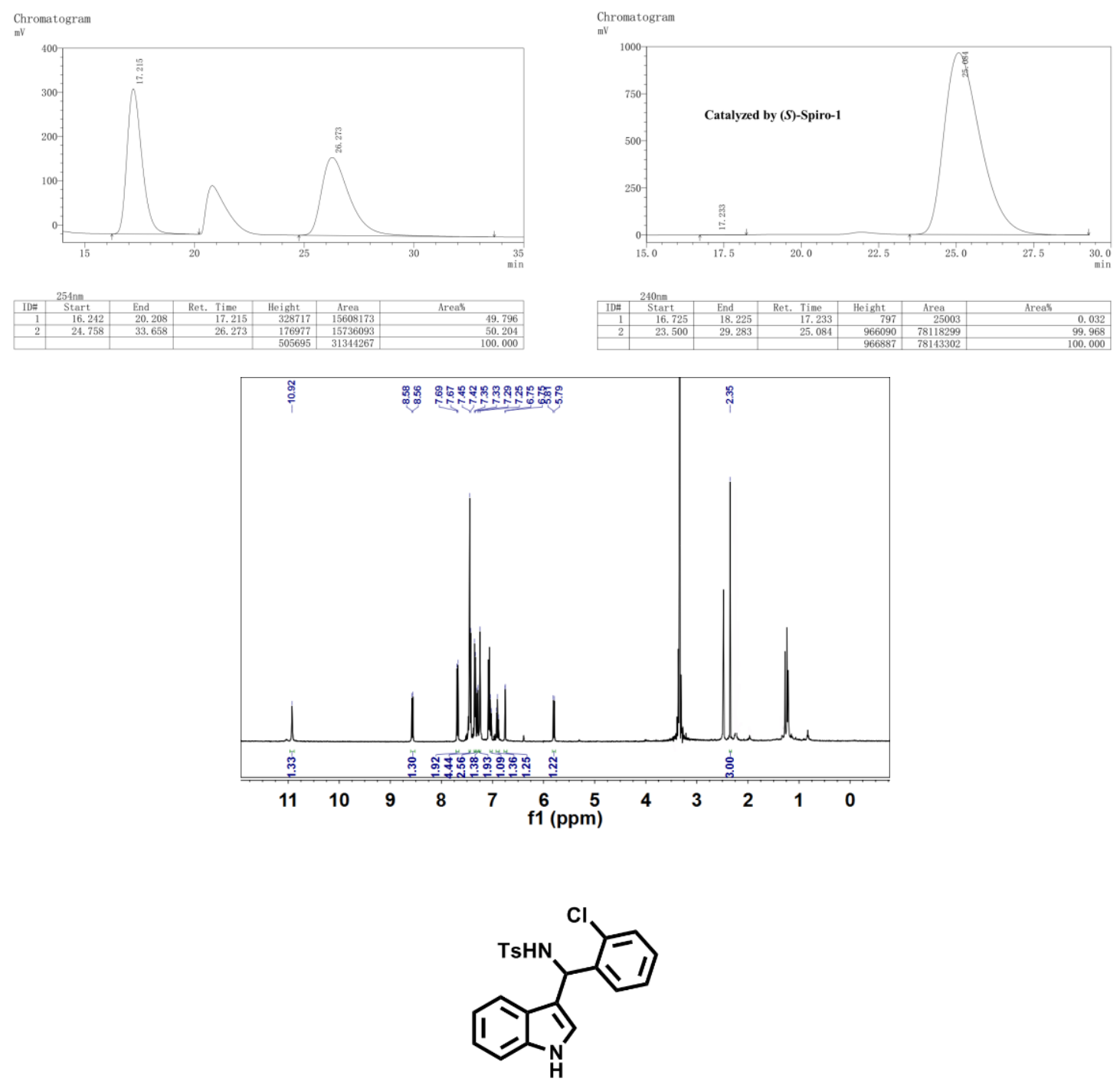

Enantiomeric excess was determined by HPLC with a chiralcel OD-H column (hexane/iPrOH $=$ $70 / 30,0.6 \mathrm{~mL} / \mathrm{min}), \mathrm{t}_{\text {minor }}=10.699 \mathrm{~min}, \mathrm{t}_{\text {major }}=22.614 \mathrm{~min}$; ee $=98 \% .{ }^{1} \mathrm{H} \mathrm{NMR}(400 \mathrm{MHz}$, $d_{6}$-DMSO) $\delta 10.91(\mathrm{~s}, 1 \mathrm{H}), 8.55(\mathrm{~d}, J=8.6 \mathrm{~Hz}, 1 \mathrm{H}), 7.69(\mathrm{~d}, J=8.2 \mathrm{~Hz}, 1 \mathrm{H}), 7.50(\mathrm{t}, J=7.5 \mathrm{~Hz}$, $2 \mathrm{H}), 7.37-7.22(\mathrm{~m}, 5 \mathrm{H}), 7.17-7.12(\mathrm{~m}, 3 \mathrm{H}), 7.06(\mathrm{dd}, J=11.1,4.0 \mathrm{~Hz}, 1 \mathrm{H}), 6.94-6.86(\mathrm{~m}$, $1 \mathrm{H}), 6.52(\mathrm{~d}, J=2.5 \mathrm{~Hz}, 1 \mathrm{H}), 6.14(\mathrm{~d}, J=8.6 \mathrm{~Hz}, 1 \mathrm{H}), 2.28(\mathrm{~s}, 3 \mathrm{H})$. 

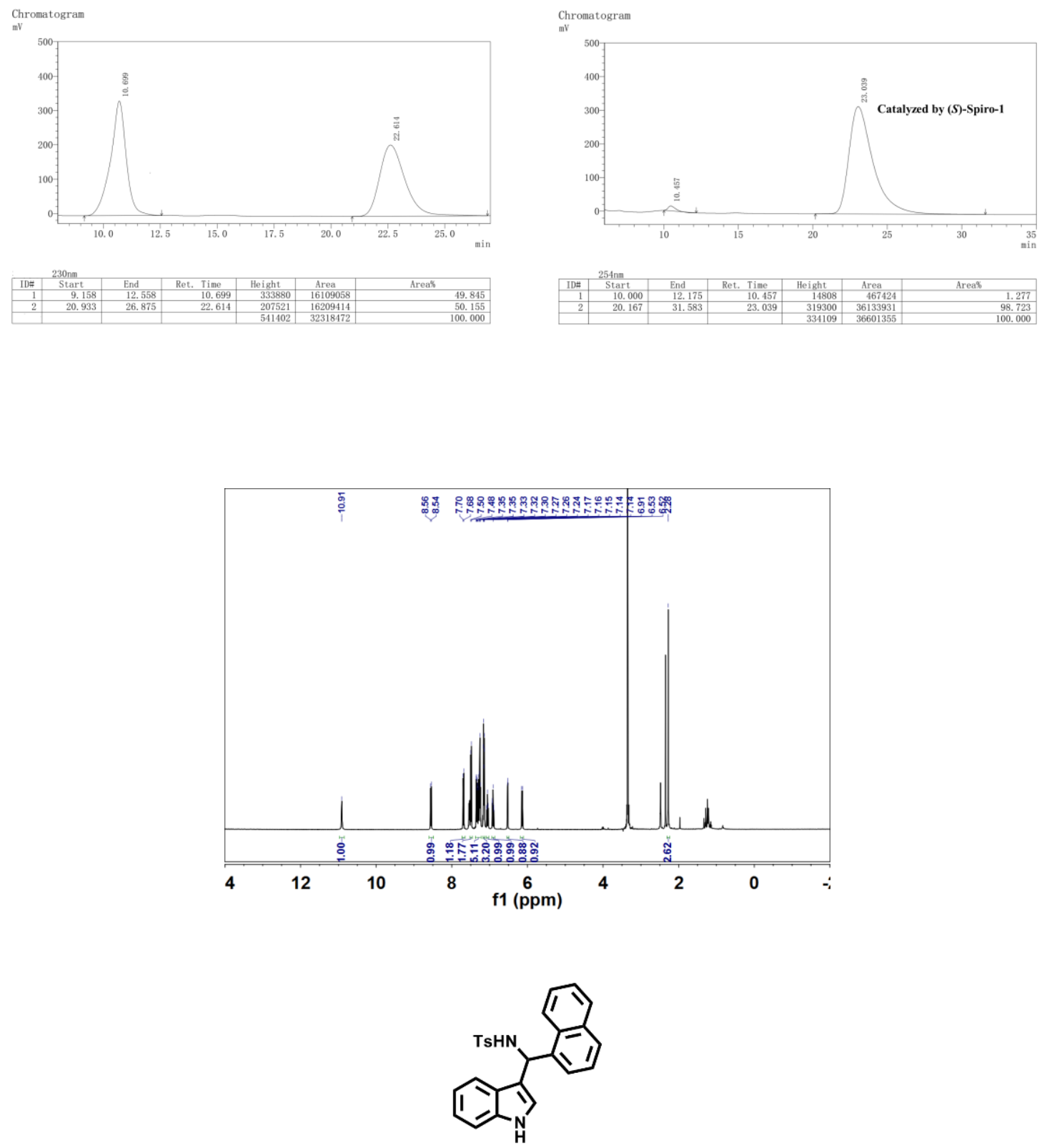

Enantiomeric excess was determined by HPLC with a chiralcel OD-H column (hexane/iPrOH $=$ $70 / 30,0.8 \mathrm{~mL} / \mathrm{min}), \mathrm{t}_{\text {minor }}=11.880 \mathrm{~min}, \mathrm{t}_{\text {major }}=21.178 \mathrm{~min}$; ee $=99 \% .{ }^{1} \mathrm{H} \mathrm{NMR}(400 \mathrm{MHz}$, $d_{6}$-DMSO) $\delta 10.83(\mathrm{t}, J=5.0 \mathrm{~Hz}, 1 \mathrm{H}), 8.57(\mathrm{~d}, J=8.2 \mathrm{~Hz}, 1 \mathrm{H}), 7.94(\mathrm{~d}, J=8.3 \mathrm{~Hz}, 1 \mathrm{H}), 7.89-$ $7.84(\mathrm{~m}, 1 \mathrm{H}), 7.75(\mathrm{~d}, J=8.2 \mathrm{~Hz}, 1 \mathrm{H}), 7.61(\mathrm{~d}, J=6.9 \mathrm{~Hz}, 1 \mathrm{H}), 7.50-7.32(\mathrm{~m}, 5 \mathrm{H}), 7.31-7.24$ $(\mathrm{m}, 1 \mathrm{H}), 7.21(\mathrm{~d}, J=8.0 \mathrm{~Hz}, 1 \mathrm{H}), 7.10-6.99(\mathrm{~m}, 3 \mathrm{H}), 6.86(\mathrm{ddd}, J=8.0,7.1,0.9 \mathrm{~Hz}, 1 \mathrm{H}), 6.59(\mathrm{~d}$, $J=2.4 \mathrm{~Hz}, 1 \mathrm{H}), 6.52(\mathrm{~d}, J=8.2 \mathrm{~Hz}, 1 \mathrm{H}), 2.25(\mathrm{~s}, 3 \mathrm{H})$. 

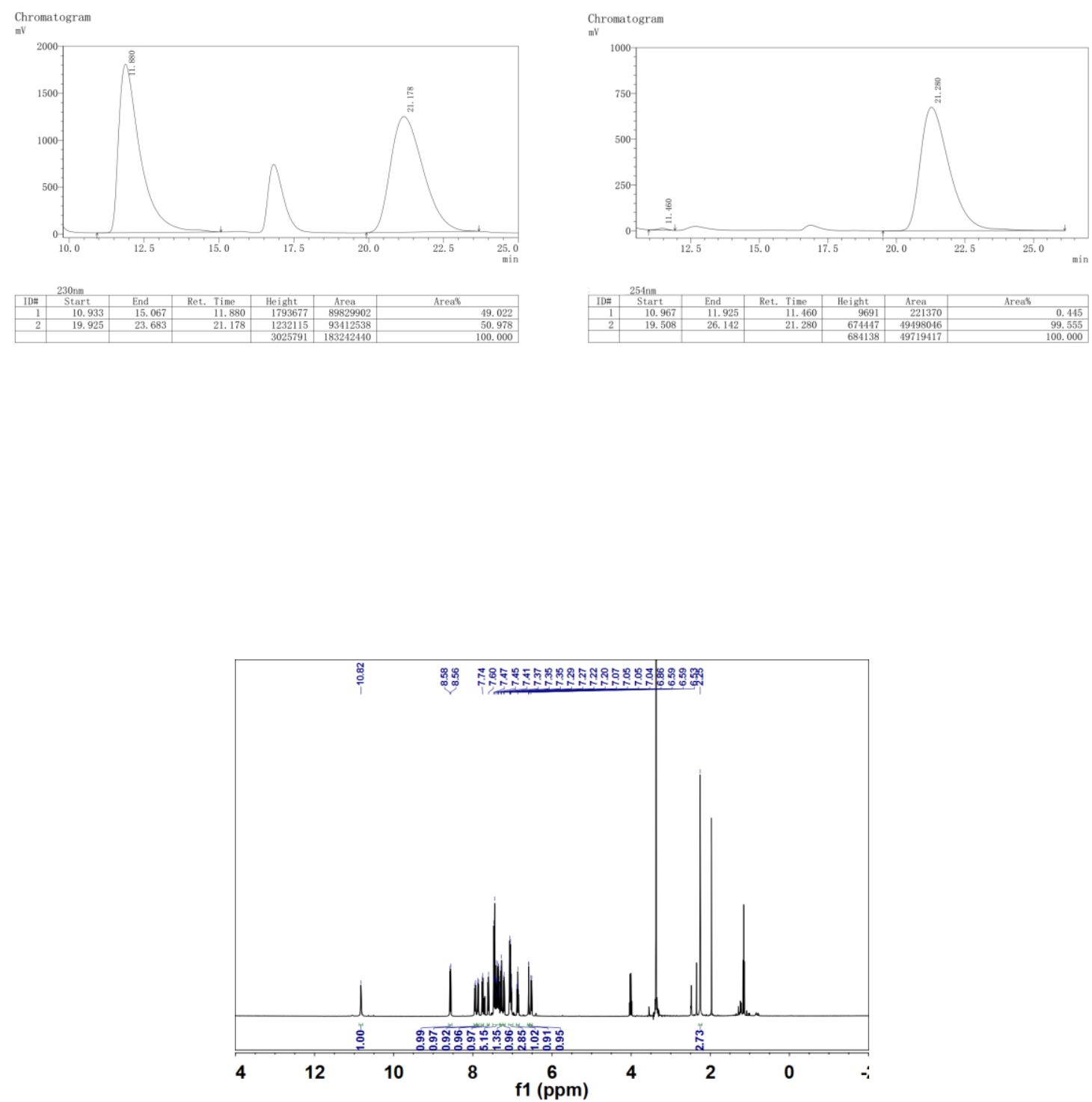

20.5 Three-Component Tandem Friedel-Crafts Reaction of Indole with Aldehydes and p-Toluenesulfonamide
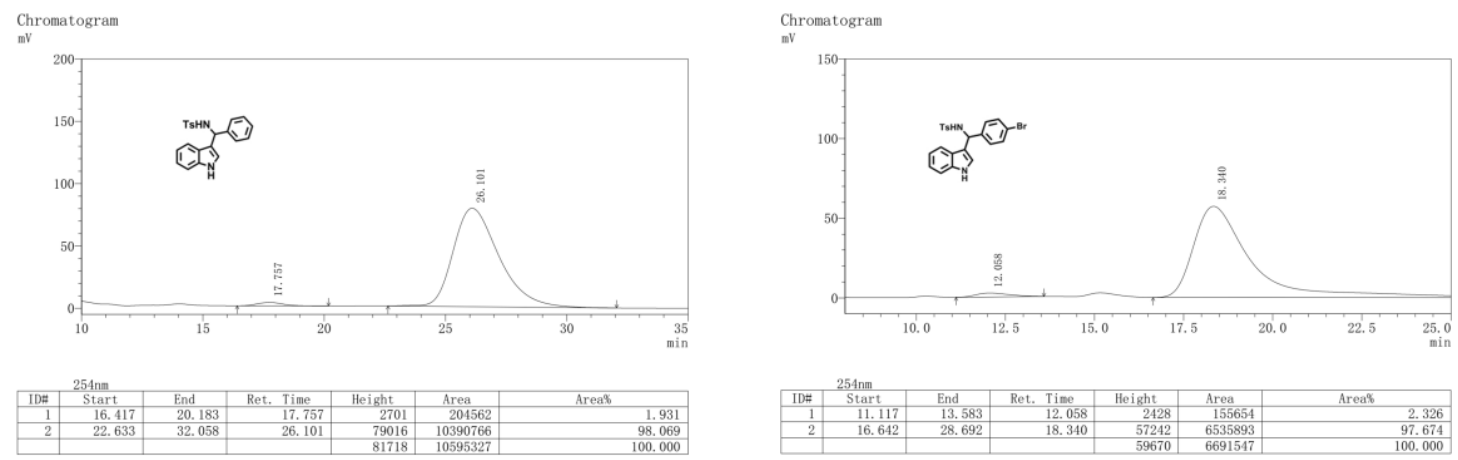
Chromatogram

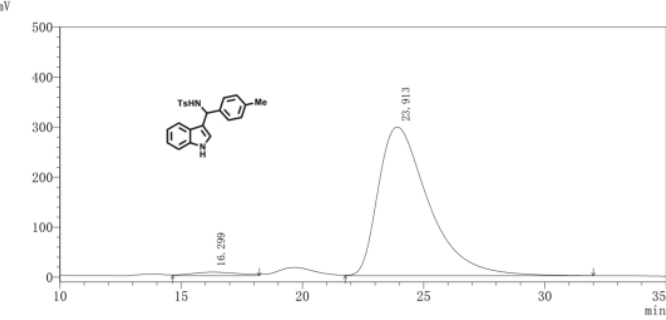

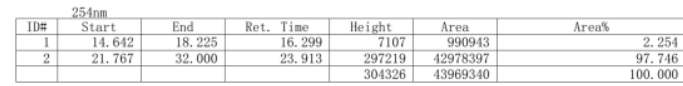

Chromatogram

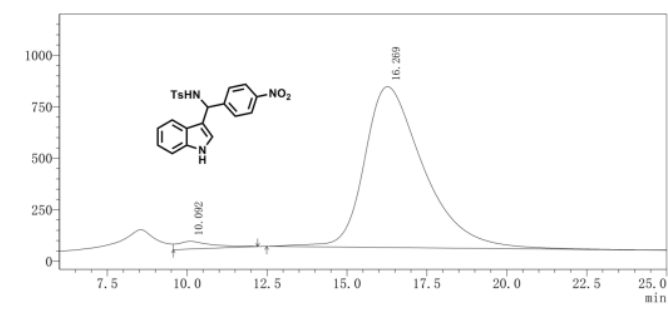

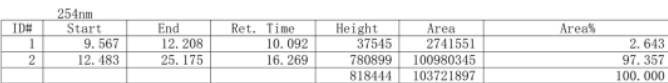
Chromatogram

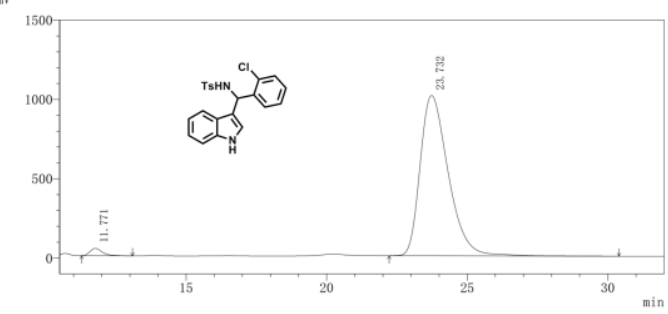

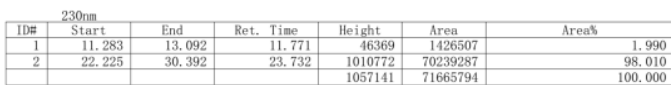

Chromatogram

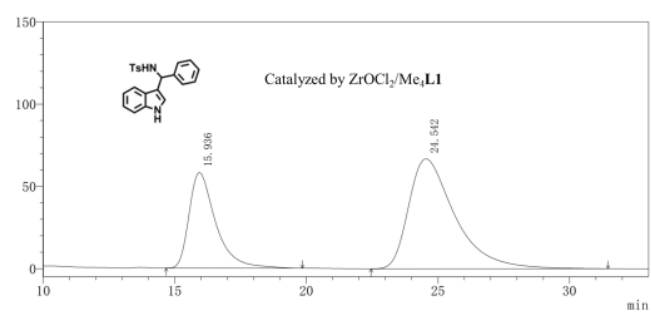

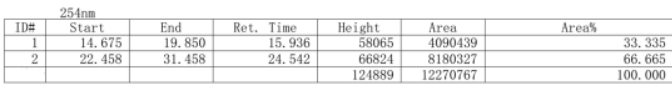

Chromatogram

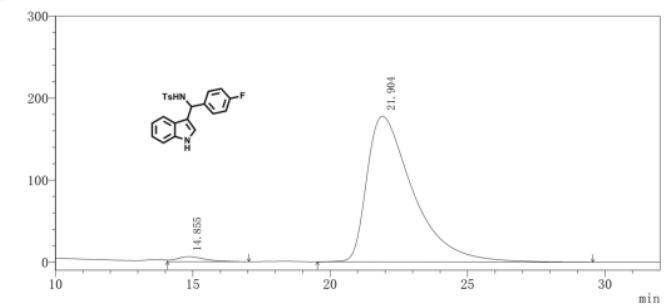

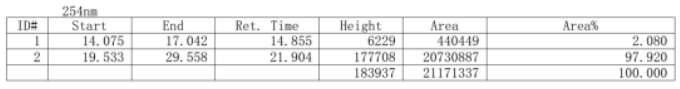

$\underset{\mathrm{n}}{\mathrm{C} \text { Chromatogram }}$

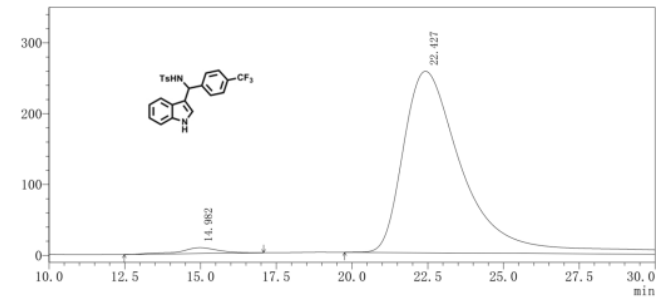

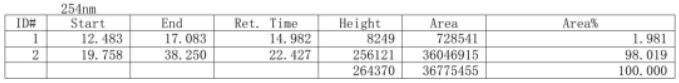

Chromatogram

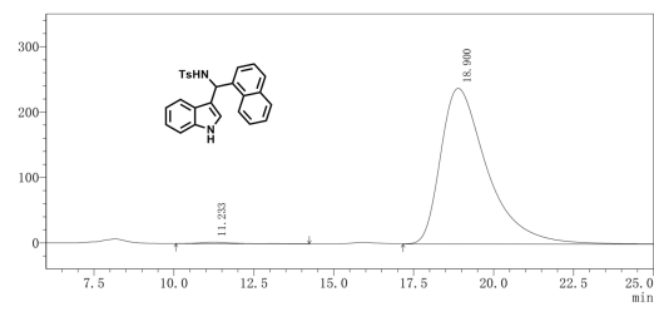

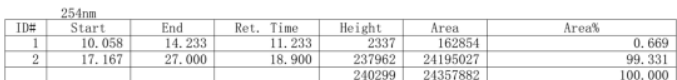

Chromatogran

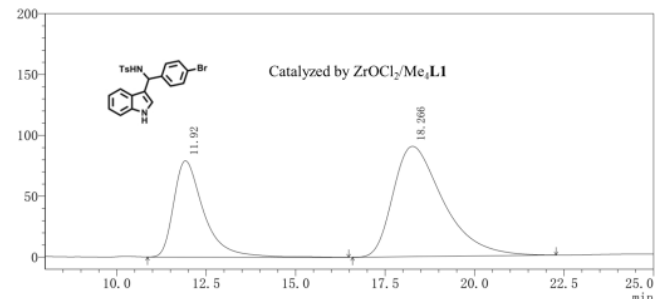

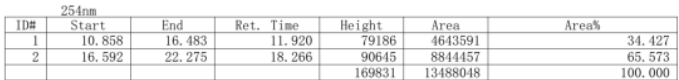



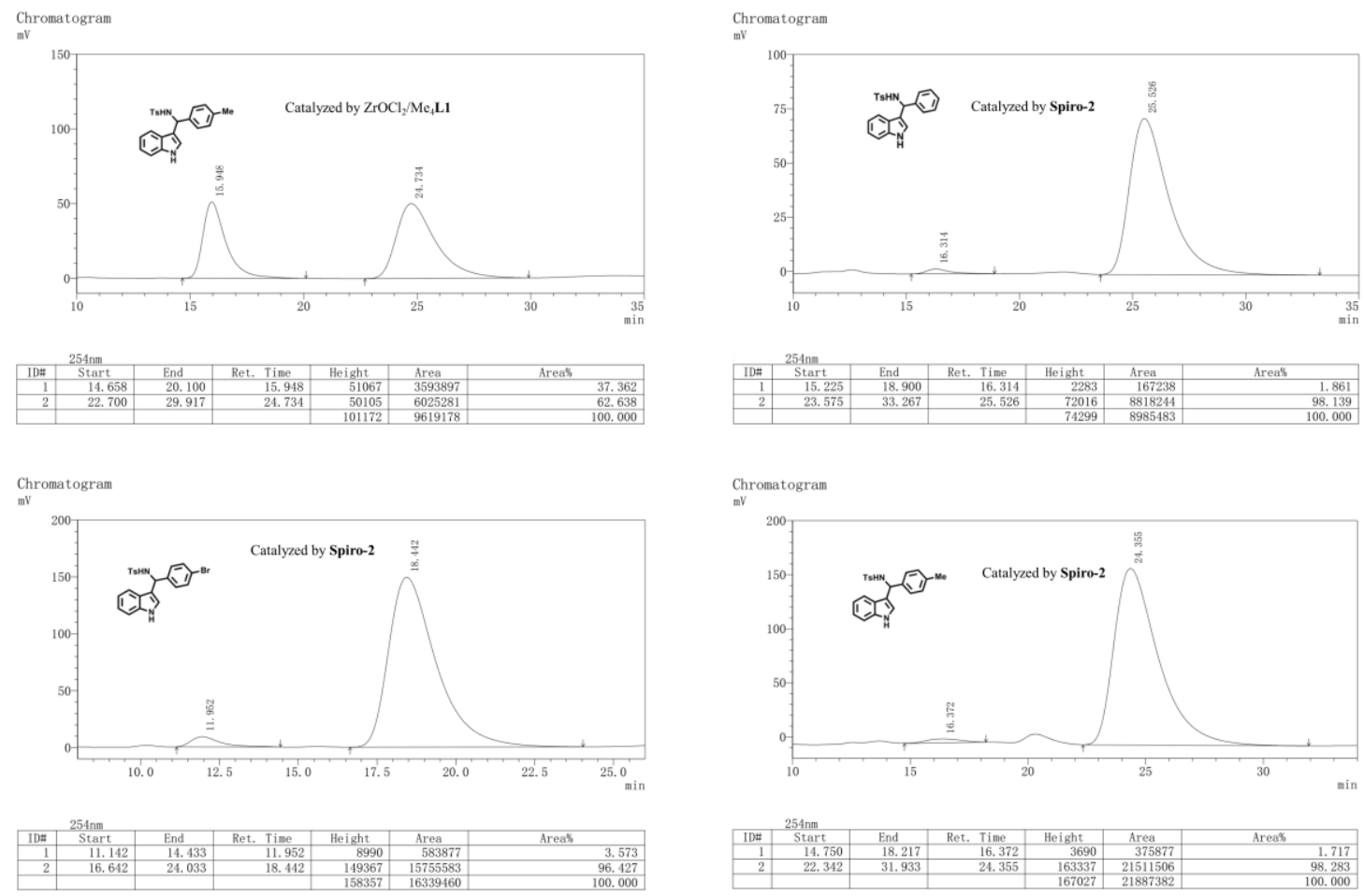

20.6 Iso-Pictet-Spengler Reaction of $o$-amino-benzylindole with Trifluoromethylated Ketones

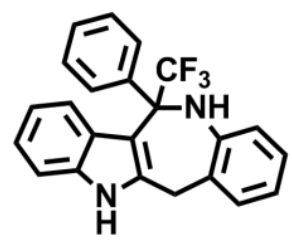

Enantiomeric excess was determined by HPLC with a chiralcel AD-H column (hexane/iPrOH $=$ 95/5, $0.8 \mathrm{~mL} / \mathrm{min}), \mathrm{t}_{\text {minor }}=17.684 \mathrm{~min}, \mathrm{t}_{\text {major }}=20.143 \mathrm{~min} ;$ ee $=90 \% .{ }^{1} \mathrm{H} \mathrm{NMR}\left(400 \mathrm{MHz}, \mathrm{CDCl}_{3}\right)$ $\delta 8.12(\mathrm{~s}, 1 \mathrm{H}), 7.36-7.24(\mathrm{~m}, 6 \mathrm{H}), 7.20(\mathrm{~d}, J=7.4 \mathrm{~Hz}, 1 \mathrm{H}), 7.11-6.97(\mathrm{~m}, 3 \mathrm{H}), 6.81(\mathrm{dd}, J=9.9$, $5.3 \mathrm{~Hz}, 1 \mathrm{H}), 6.63(\mathrm{~d}, J=8.2 \mathrm{~Hz}, 1 \mathrm{H}), 6.50(\mathrm{~d}, J=7.6 \mathrm{~Hz}, 1 \mathrm{H}), 4.51(\mathrm{~d}, J=15.1 \mathrm{~Hz}, 1 \mathrm{H}), 3.95-$ $3.80(\mathrm{~m}, 2 \mathrm{H})$.
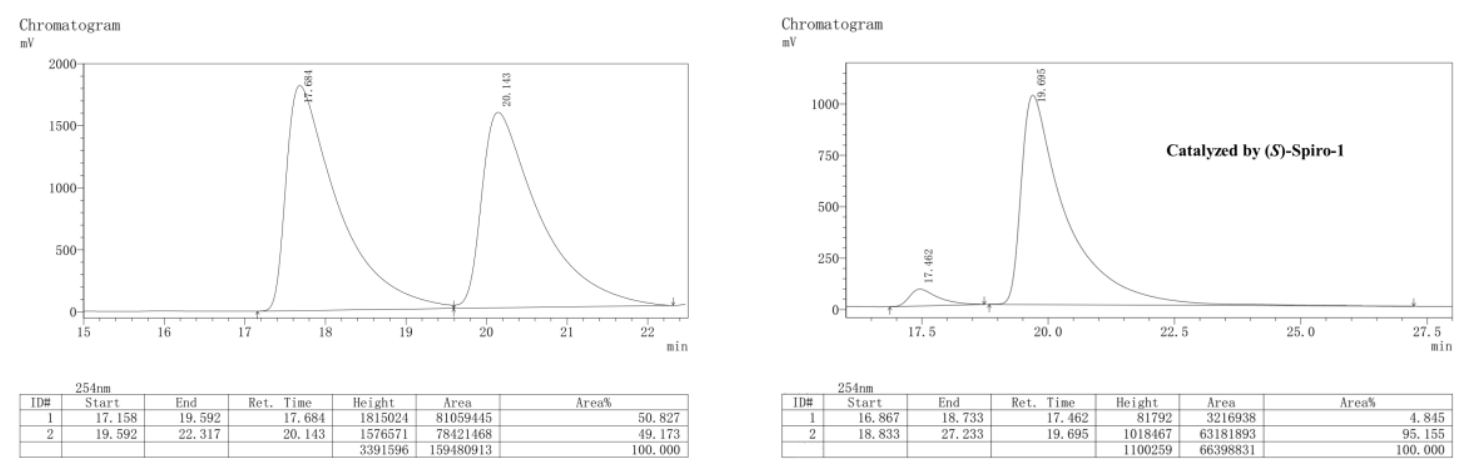

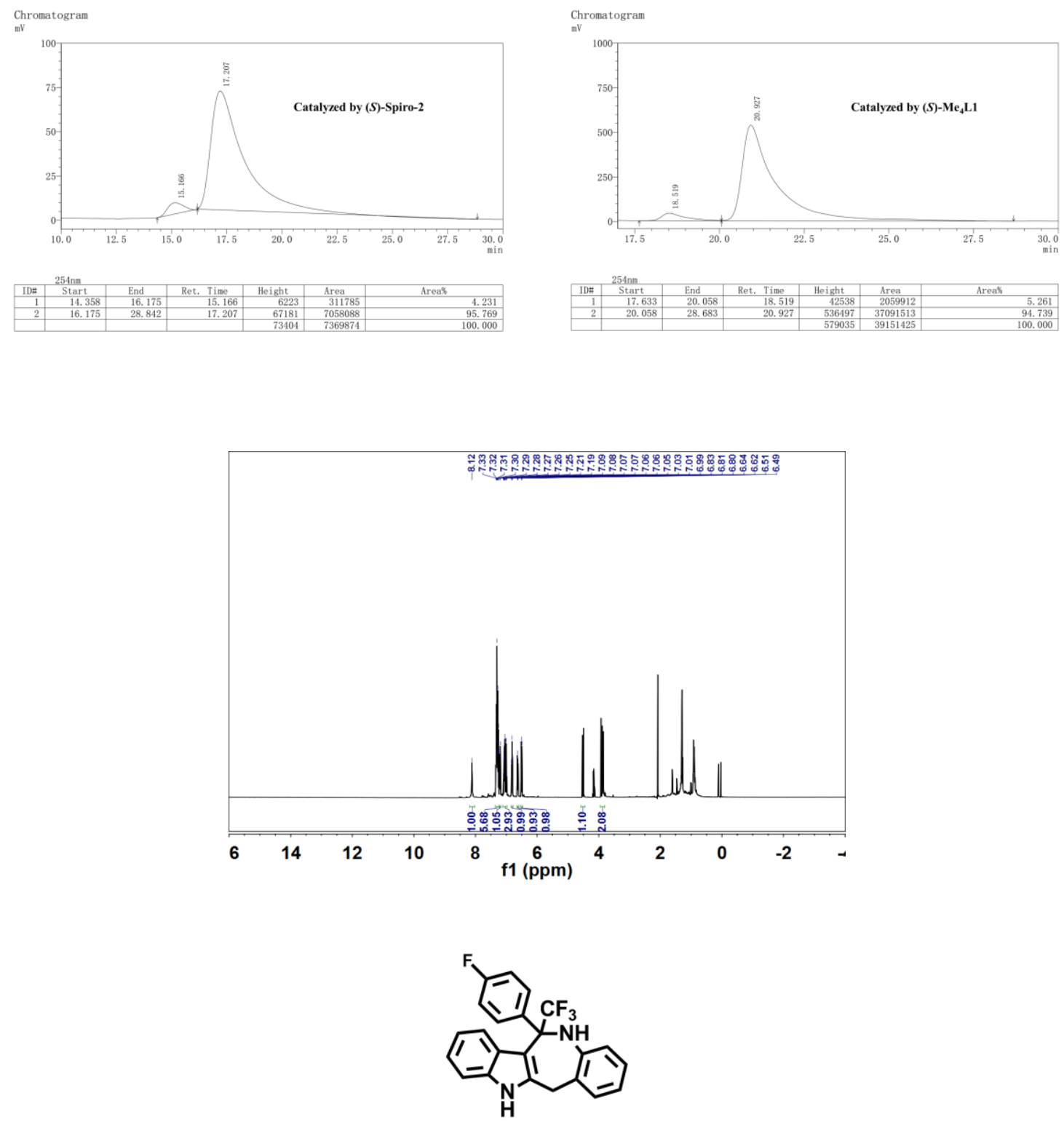

Enantiomeric excess was determined by HPLC with a chiralcel AD-H column (hexane/iPrOH = $95 / 5,0.8 \mathrm{~mL} / \mathrm{min}), \mathrm{t}_{\text {minor }}=15.955 \mathrm{~min}, \mathrm{t}_{\text {major }}=20.930 \mathrm{~min} ; \mathrm{ee}=90 \% .{ }^{1} \mathrm{H} \mathrm{NMR}\left(400 \mathrm{MHz}, \mathrm{CDCl}_{3}\right)$ $\delta 8.13(\mathrm{~s}, 1 \mathrm{H}), 7.30-7.22(\mathrm{~m}, 3 \mathrm{H}), 7.19(\mathrm{~d}, J=7.2 \mathrm{~Hz}, 1 \mathrm{H}), 7.09-6.90(\mathrm{~m}, 5 \mathrm{H}), 6.85-6.78(\mathrm{~m}$, $1 \mathrm{H}), 6.59(\mathrm{~d}, J=8.2 \mathrm{~Hz}, 1 \mathrm{H}), 6.47(\mathrm{~d}, J=7.5 \mathrm{~Hz}, 1 \mathrm{H}), 4.51(\mathrm{~d}, J=15.0 \mathrm{~Hz}, 1 \mathrm{H}), 3.95-3.83(\mathrm{~m}$, $1 \mathrm{H}), 3.77(\mathrm{~s}, 1 \mathrm{H})$.
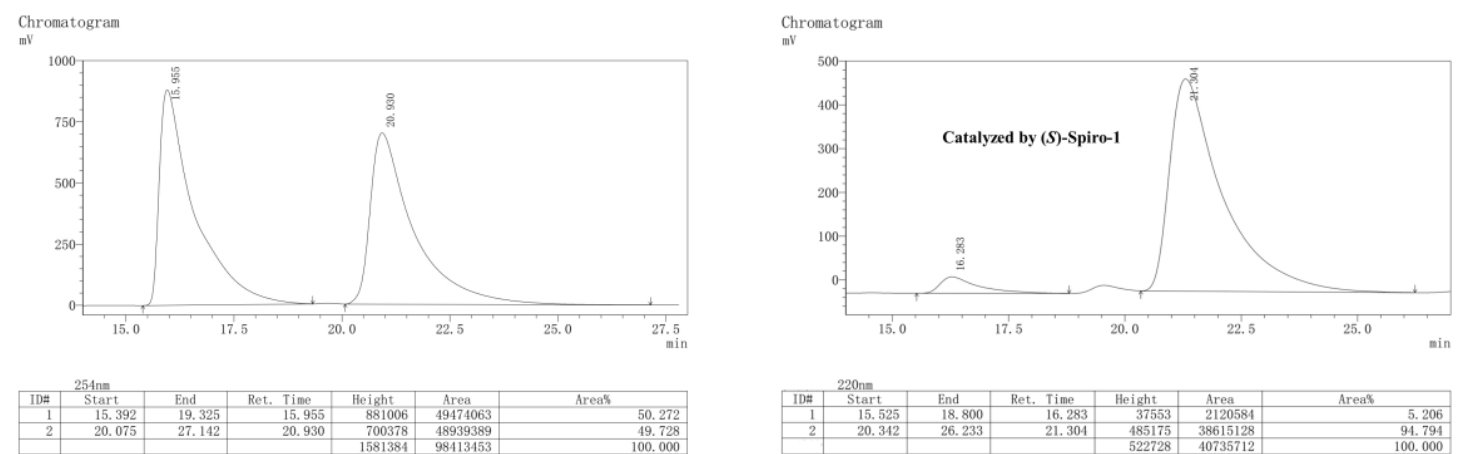
$\underset{\mathrm{mV}}{\mathrm{M} \text { Chrotogram }}$
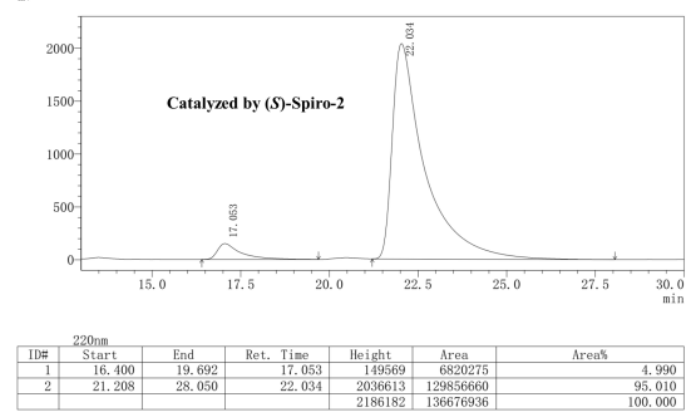

$\underset{\mathrm{mV}}{\text { Chromatogram }}$
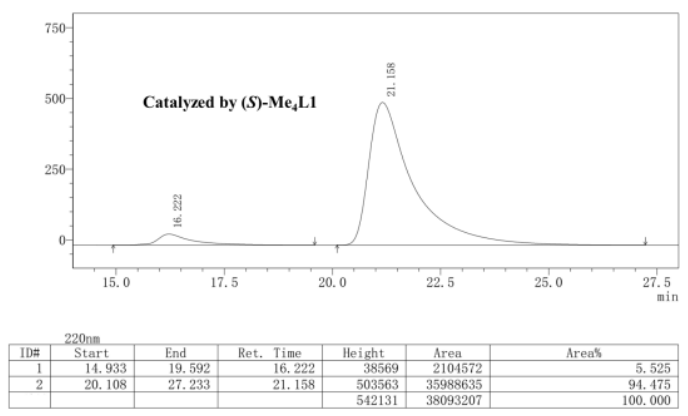
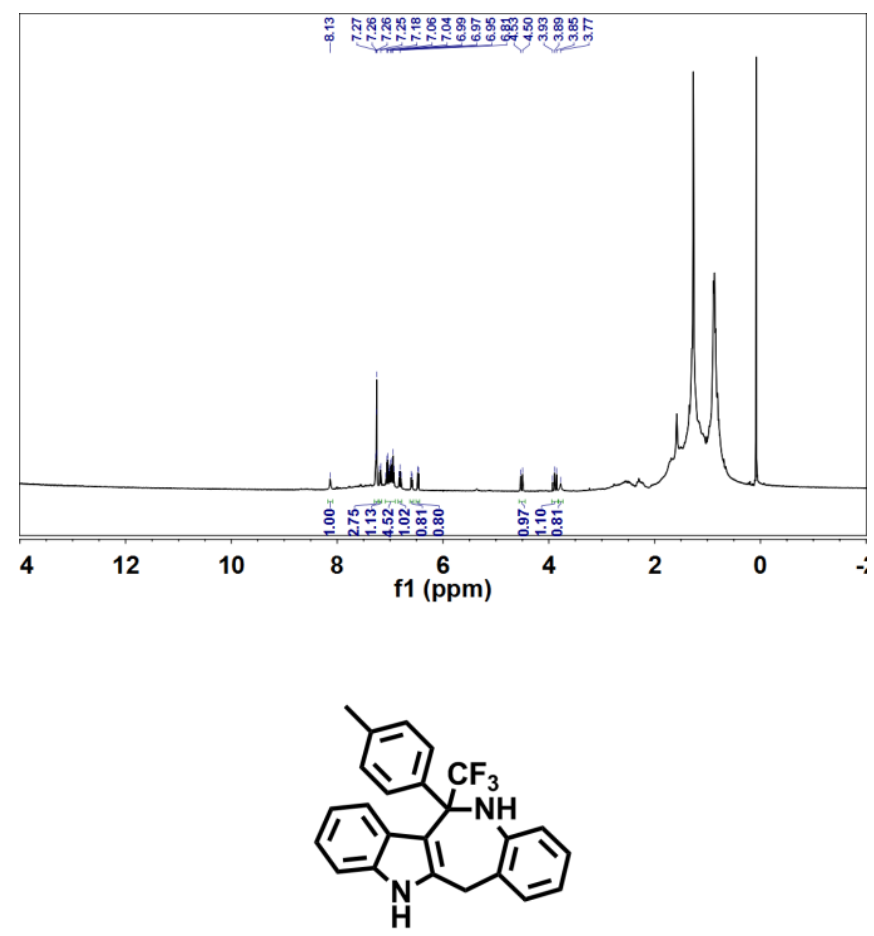

Enantiomeric excess was determined by HPLC with a chiralcel AD-H column (hexane/iPrOH $=$ $95 / 5,0.8 \mathrm{~mL} / \mathrm{min}), \mathrm{t}_{\text {minor }}=15.840 \mathrm{~min}, \mathrm{t}_{\text {major }}=19.239 \mathrm{~min}$; ee $=89 \% .{ }^{1} \mathrm{H} \mathrm{NMR}\left(400 \mathrm{MHz}, \mathrm{CDCl}_{3}\right)$ $\delta 8.10(\mathrm{~s}, 1 \mathrm{H}), 7.25(\mathrm{t}, J=5.0 \mathrm{~Hz}, 1 \mathrm{H}), 7.19(\mathrm{~d}, J=7.9 \mathrm{~Hz}, 3 \mathrm{H}), 7.10-6.93(\mathrm{~m}, 5 \mathrm{H}), 6.80(\mathrm{t}, J=$ $7.6 \mathrm{~Hz}, 1 \mathrm{H}), 6.63(\mathrm{~d}, J=8.3 \mathrm{~Hz}, 1 \mathrm{H}), 6.51(\mathrm{~d}, J=7.6 \mathrm{~Hz}, 1 \mathrm{H}), 4.47(\mathrm{~d}, J=15.1 \mathrm{~Hz}, 1 \mathrm{H}), 3.91(\mathrm{~d}$, $J=15.1 \mathrm{~Hz}, 1 \mathrm{H}), 3.77(\mathrm{~s}, 1 \mathrm{H}), 2.32(\mathrm{~s}, 3 \mathrm{H})$.
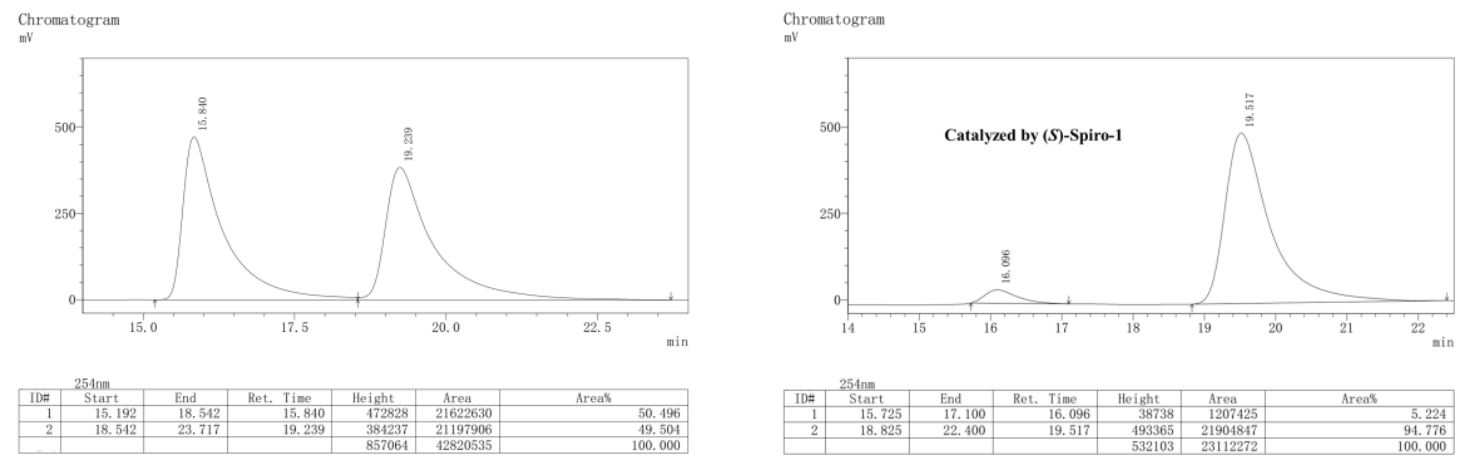

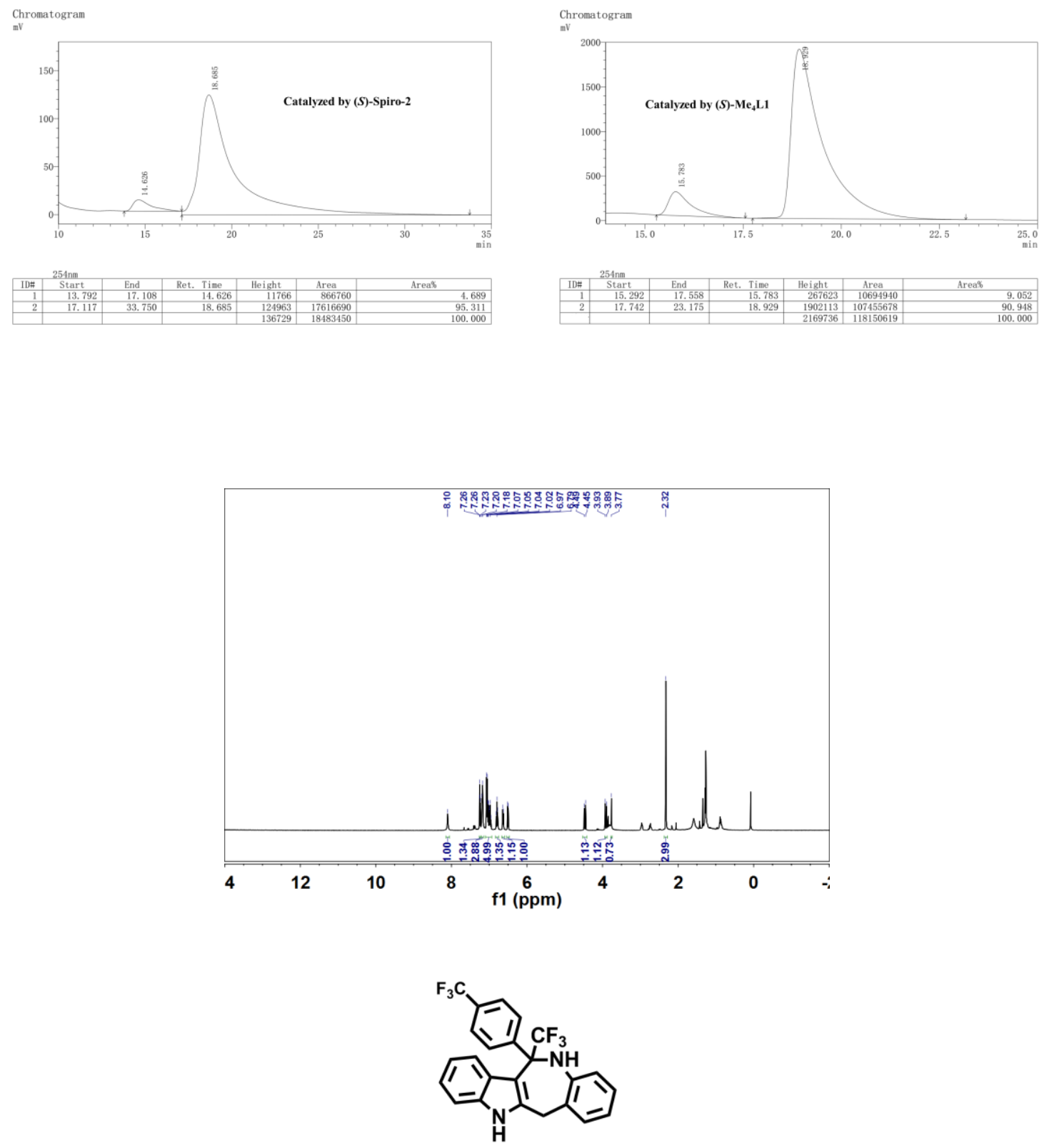

Enantiomeric excess was determined by HPLC with a chiralcel AD-H column (hexane/iPrOH $=$ $95 / 5,0.8 \mathrm{~mL} / \mathrm{min}), \mathrm{t}_{\text {minor }}=13.691 \mathrm{~min}, \mathrm{t}_{\text {major }}=16.590 \mathrm{~min}$; ee $=96 \% .{ }^{1} \mathrm{H} \mathrm{NMR}\left(400 \mathrm{MHz}, \mathrm{CDCl}_{3}\right)$ $\delta 8.23-8.12(\mathrm{~m}, 1 \mathrm{H}), 7.52(\mathrm{~d}, J=8.5 \mathrm{~Hz}, 2 \mathrm{H}), 7.39(\mathrm{~d}, J=8.1 \mathrm{~Hz}, 2 \mathrm{H}), 7.30-7.24(\mathrm{~m}, 1 \mathrm{H}), 7.20$ $(\mathrm{d}, J=7.1 \mathrm{~Hz}, 1 \mathrm{H}), 7.03(\mathrm{dq}, J=14.7,7.3 \mathrm{~Hz}, 3 \mathrm{H}), 6.81(\mathrm{t}, J=7.6 \mathrm{~Hz}, 1 \mathrm{H}), 6.54(\mathrm{~d}, J=8.2 \mathrm{~Hz}$, $1 \mathrm{H}), 6.42(\mathrm{~d}, J=7.5 \mathrm{~Hz}, 1 \mathrm{H}), 4.57(\mathrm{~d}, J=15.0 \mathrm{~Hz}, 1 \mathrm{H}), 3.90-3.74(\mathrm{~m}, 2 \mathrm{H})$.
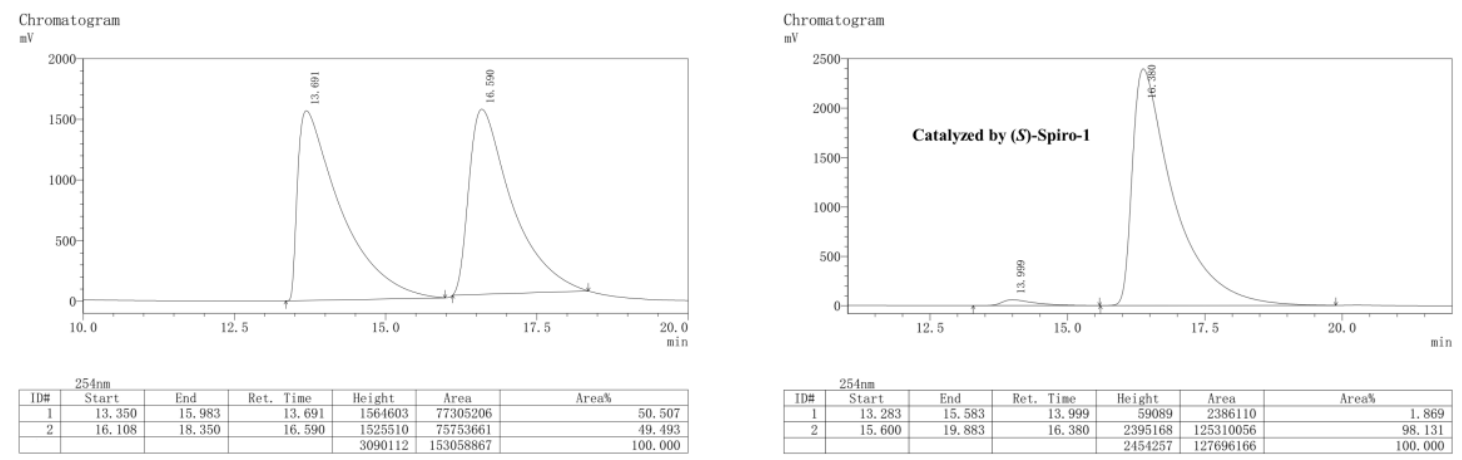

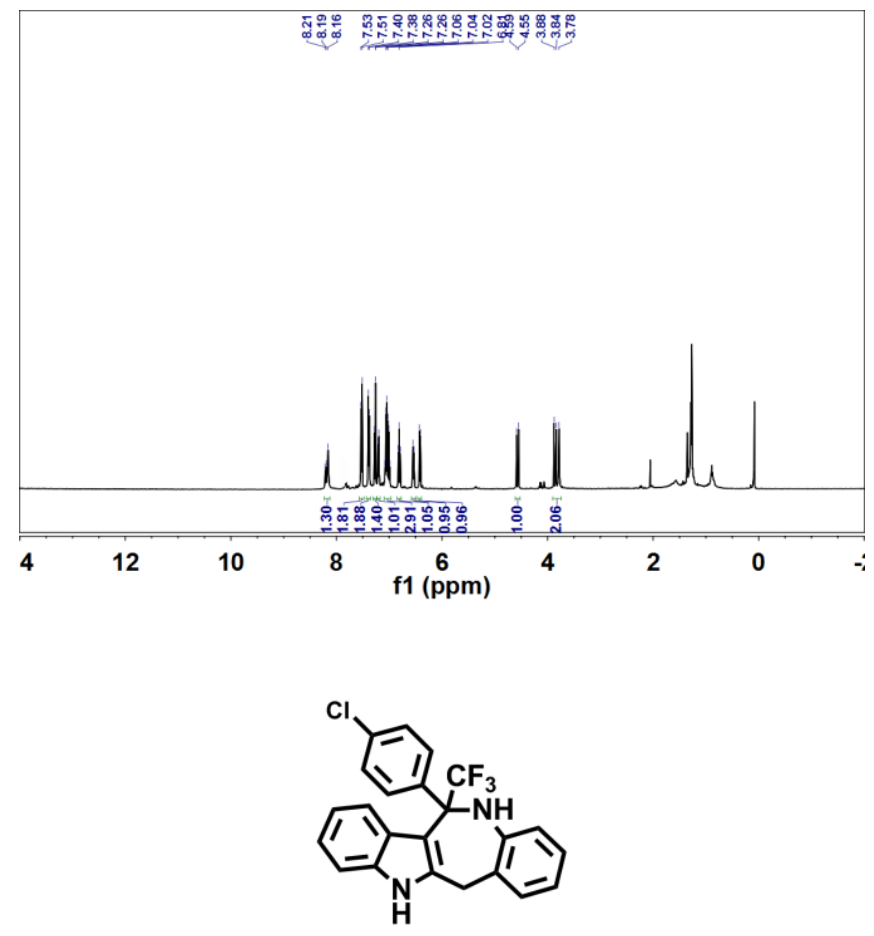

Enantiomeric excess was determined by HPLC with a chiralcel AD-H column (hexane/iPrOH $=$ 95/5, $0.8 \mathrm{~mL} / \mathrm{min}), \mathrm{t}_{\text {minor }}=17.075 \mathrm{~min}, \mathrm{t}_{\text {major }}=20.137 \mathrm{~min}$; ee $=96 \% .{ }^{1} \mathrm{H} \mathrm{NMR}\left(400 \mathrm{MHz}, \mathrm{CDCl}_{3}\right)$ $\delta 8.15(\mathrm{~s}, 1 \mathrm{H}), 7.23(\mathrm{ddd}, J=18.7,10.8,5.8 \mathrm{~Hz}, 6 \mathrm{H}), 7.03(\mathrm{dqd}, J=16.0,7.4,1.4 \mathrm{~Hz}, 3 \mathrm{H}), 6.82$ (ddd, $J=8.1,7.1,1.0 \mathrm{~Hz}, 1 \mathrm{H}), 6.60(\mathrm{~d}, J=8.1 \mathrm{~Hz}, 1 \mathrm{H}), 6.46(\mathrm{~d}, J=7.5 \mathrm{~Hz}, 1 \mathrm{H}), 4.54(\mathrm{~d}, J=15.1$ $\mathrm{Hz}, 1 \mathrm{H}), 3.85(\mathrm{~d}, J=15.1 \mathrm{~Hz}, 1 \mathrm{H})$.
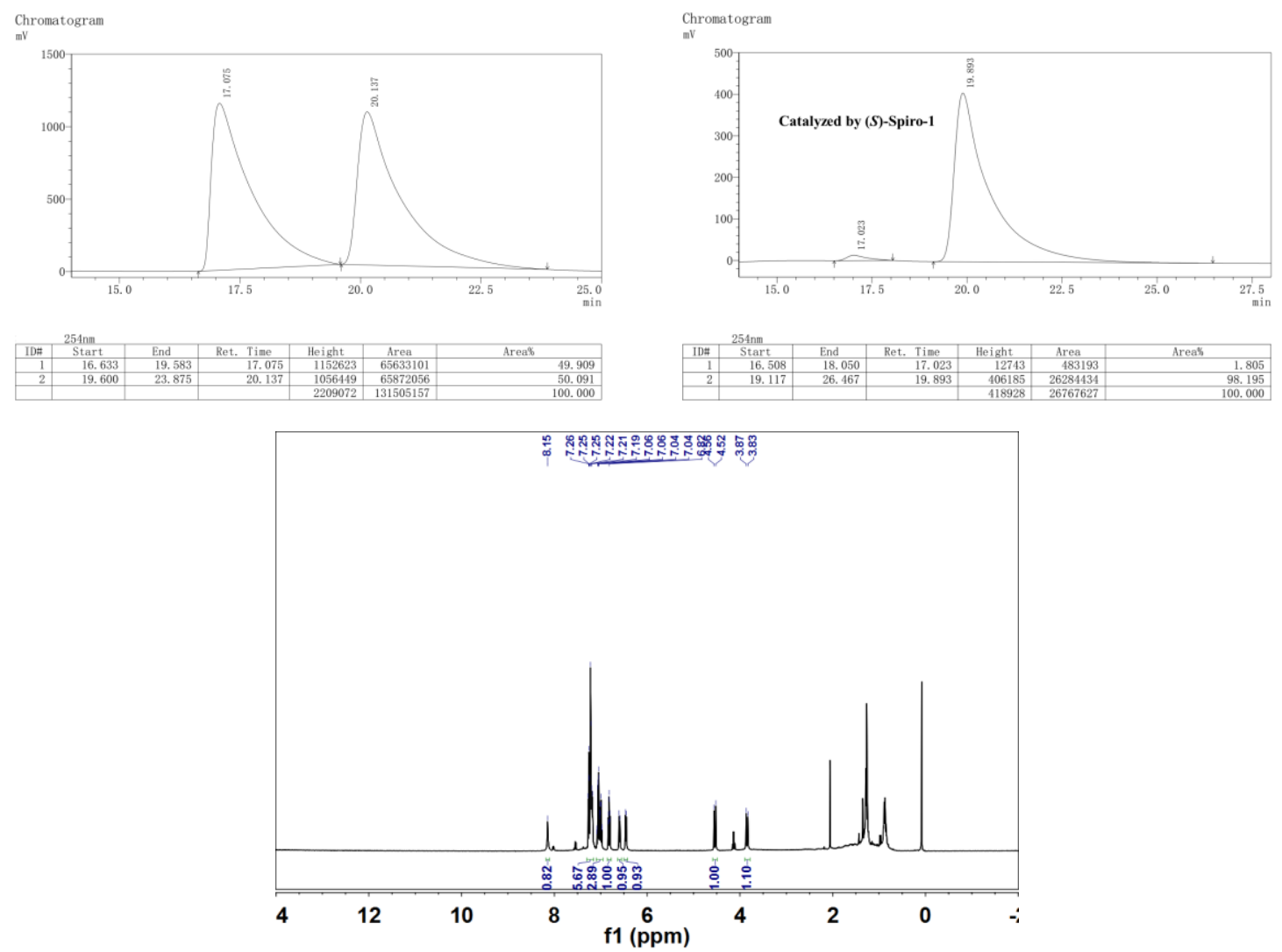


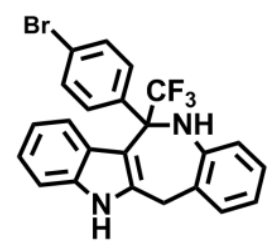

Enantiomeric excess was determined by HPLC with a chiralcel AD-H column (hexane/ $\mathrm{PrOH}=$ 95/5, $0.8 \mathrm{~mL} / \mathrm{min}), \mathrm{t}_{\text {minor }}=17.288 \mathrm{~min}, \mathrm{t}_{\text {major }}=20.419 \mathrm{~min}$; ee $=96 \% .{ }^{1} \mathrm{H} \mathrm{NMR}\left(400 \mathrm{MHz}, \mathrm{CDCl}_{3}\right)$ $\delta 8.12(\mathrm{~s}, 1 \mathrm{H}), 7.37-7.22(\mathrm{~m}, 5 \mathrm{H}), 7.20(\mathrm{~d}, J=7.4 \mathrm{~Hz}, 1 \mathrm{H}), 7.12-6.96(\mathrm{~m}, 3 \mathrm{H}), 6.86-6.77(\mathrm{~m}$, $1 \mathrm{H}), 6.63(\mathrm{~d}, J=8.2 \mathrm{~Hz}, 1 \mathrm{H}), 6.50(\mathrm{~d}, J=7.6 \mathrm{~Hz}, 1 \mathrm{H}), 4.51(\mathrm{~d}, J=15.1 \mathrm{~Hz}, 1 \mathrm{H}), 3.95-3.82(\mathrm{~m}$, $2 \mathrm{H})$.
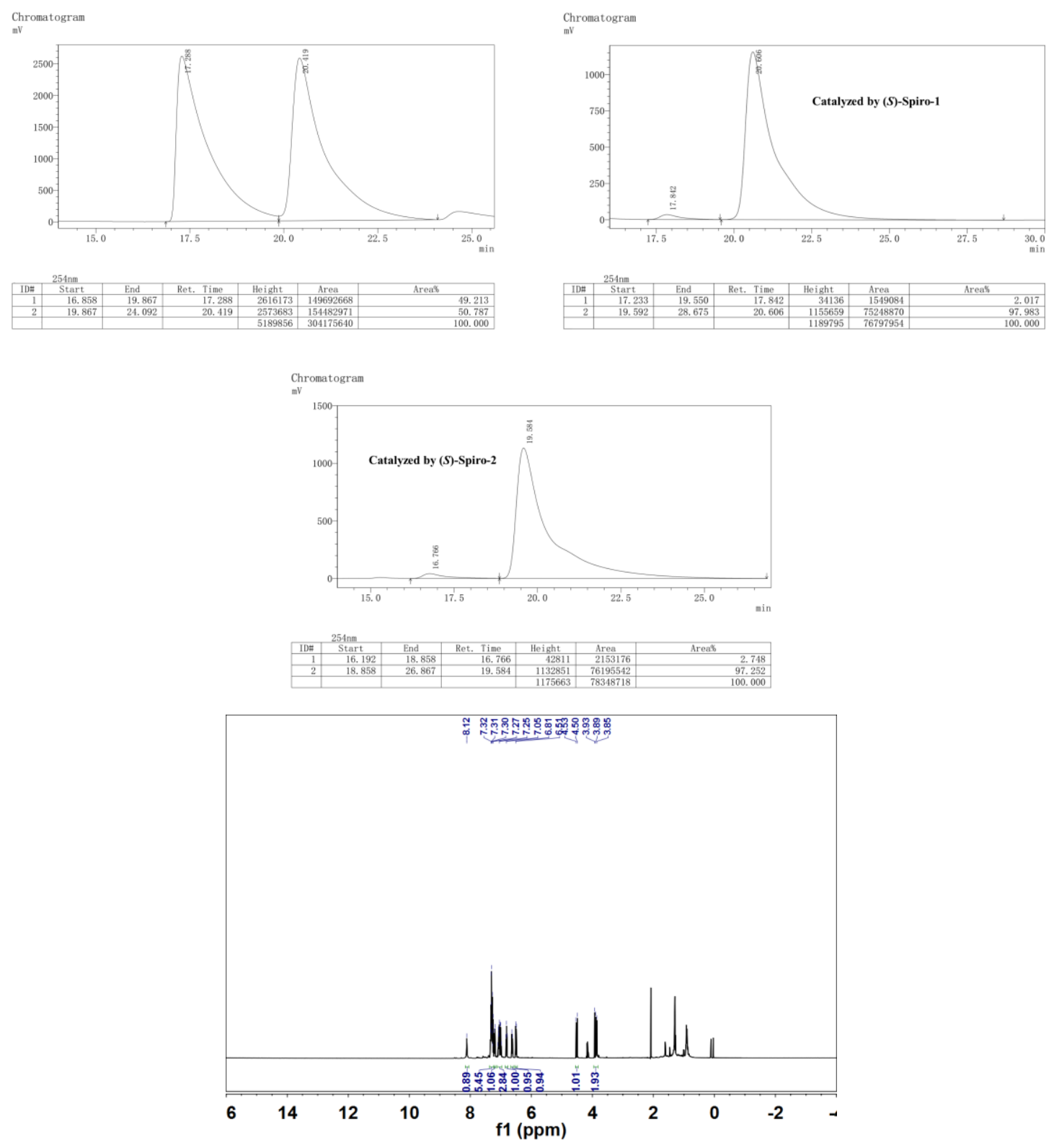


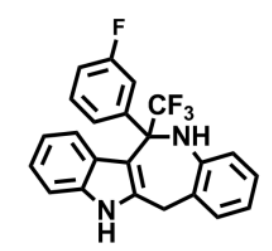

Enantiomeric excess was determined by HPLC with a chiralcel AD-H column (hexane/ $\mathrm{PrOH}=$ $95 / 5,0.8 \mathrm{~mL} / \mathrm{min}), \mathrm{t}_{\text {minor }}=21.176 \mathrm{~min}, \mathrm{t}_{\text {major }}=25.852 \mathrm{~min} ;$ ee $=90 \% .{ }^{1} \mathrm{H} \mathrm{NMR}\left(400 \mathrm{MHz}, \mathrm{CDCl}_{3}\right)$ $\delta 8.14(\mathrm{~s}, 1 \mathrm{H}), 7.23(\mathrm{ddd}, J=18.0,11.9,7.3 \mathrm{~Hz}, 3 \mathrm{H}), 7.11-6.95(\mathrm{~m}, 6 \mathrm{H}), 6.82(\mathrm{t}, J=7.6 \mathrm{~Hz}, 1 \mathrm{H})$, $6.63(\mathrm{~d}, J=8.2 \mathrm{~Hz}, 1 \mathrm{H}), 6.47(\mathrm{~d}, J=7.6 \mathrm{~Hz}, 1 \mathrm{H}), 4.53(\mathrm{~d}, J=15.1 \mathrm{~Hz}, 1 \mathrm{H}), 3.86(\mathrm{~d}, J=15.1 \mathrm{~Hz}$, $1 \mathrm{H})$.
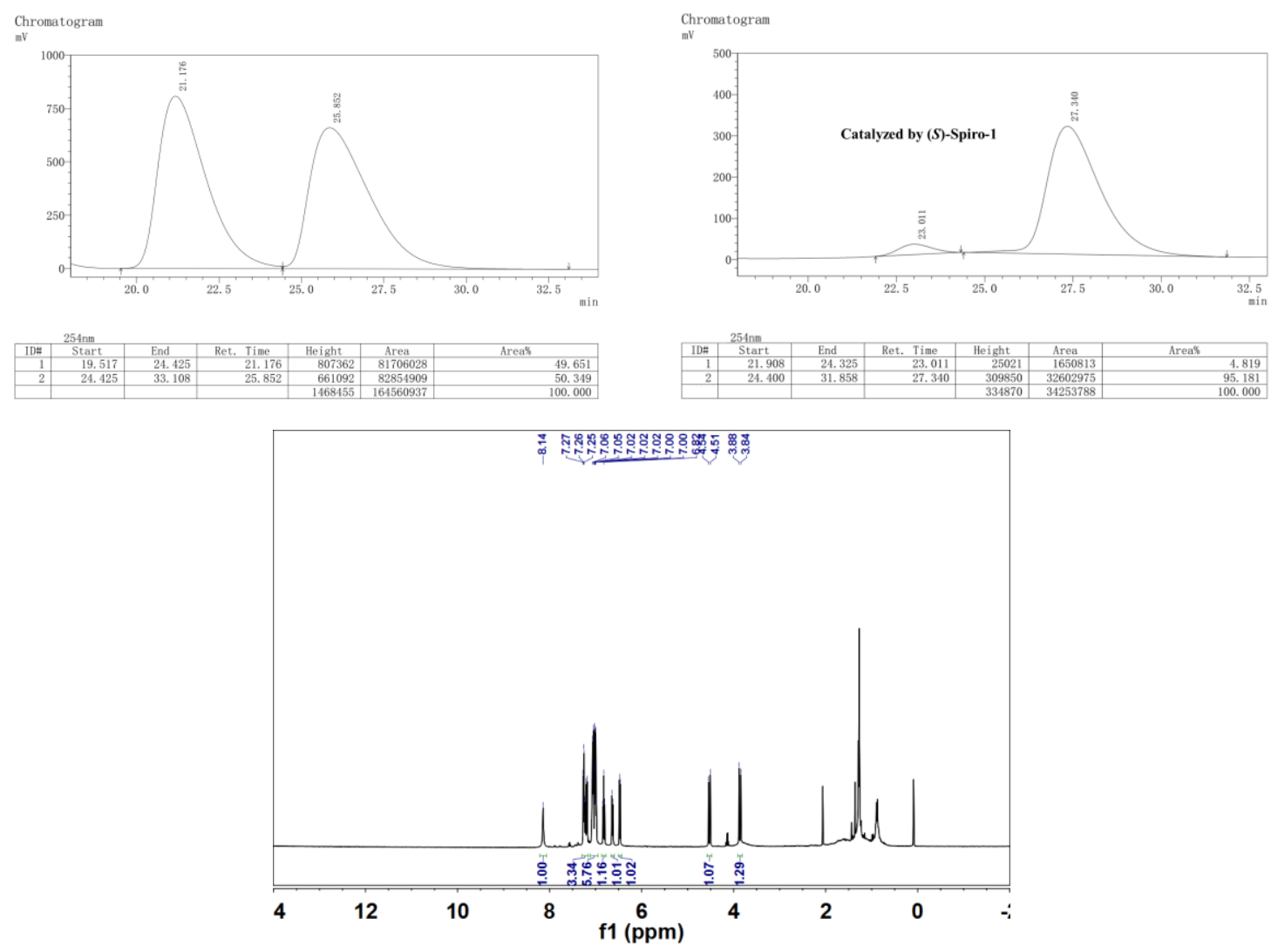

20.7 HPLC spectra of recycle experiment for Spiro-1 catalyzed acetalization reaction of 2-aminobenzamide with 4-bromobenzaldehyde
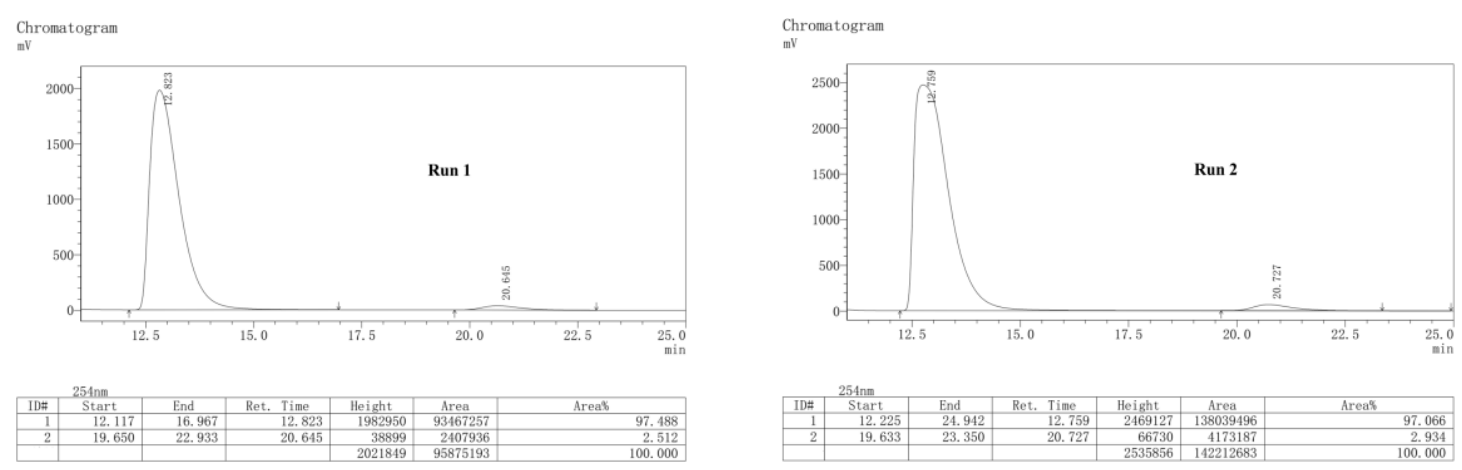
$\underset{\mathrm{mV}}{\mathrm{M}} \mathrm{Chratogram}$
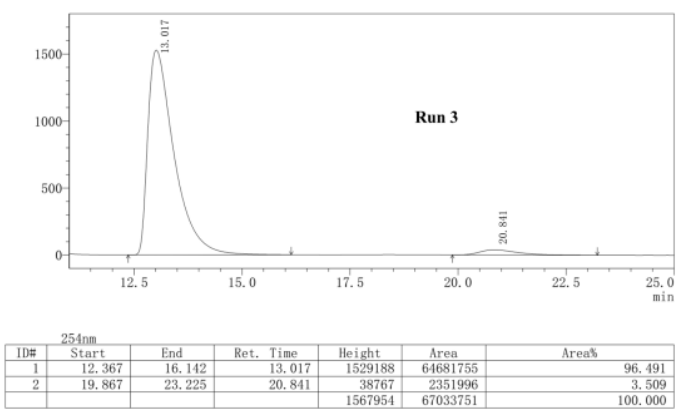

$\underset{\mathrm{N}}{\mathrm{C} \text { Chromatogram }}$
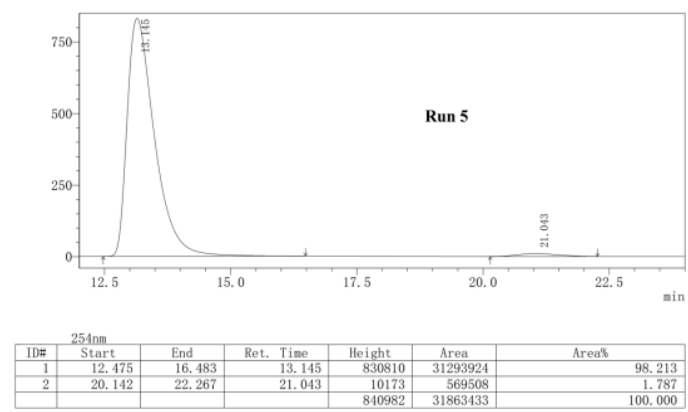

Chromatogram
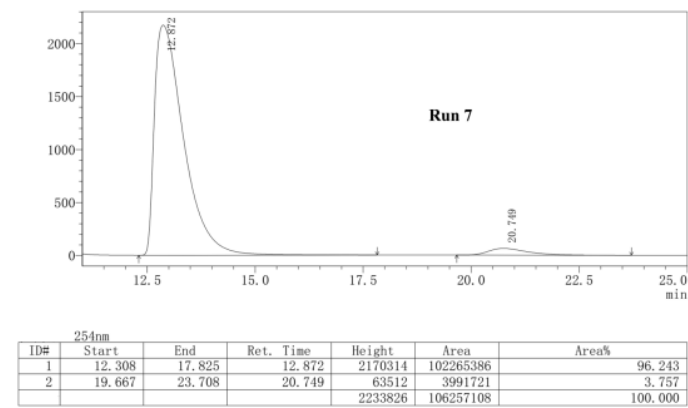

Chromatogram

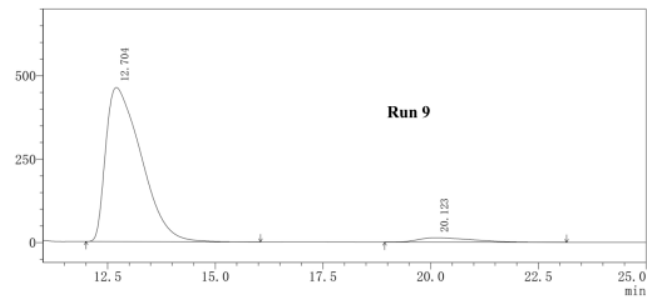

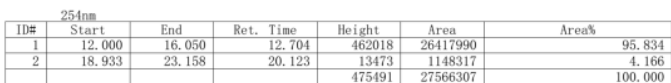

Chromatogram
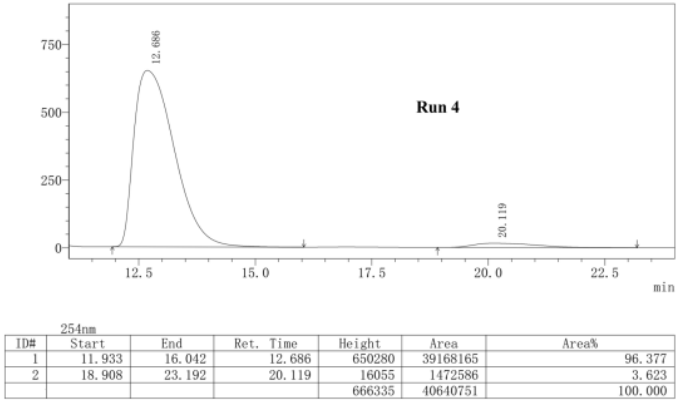

Chromatogram
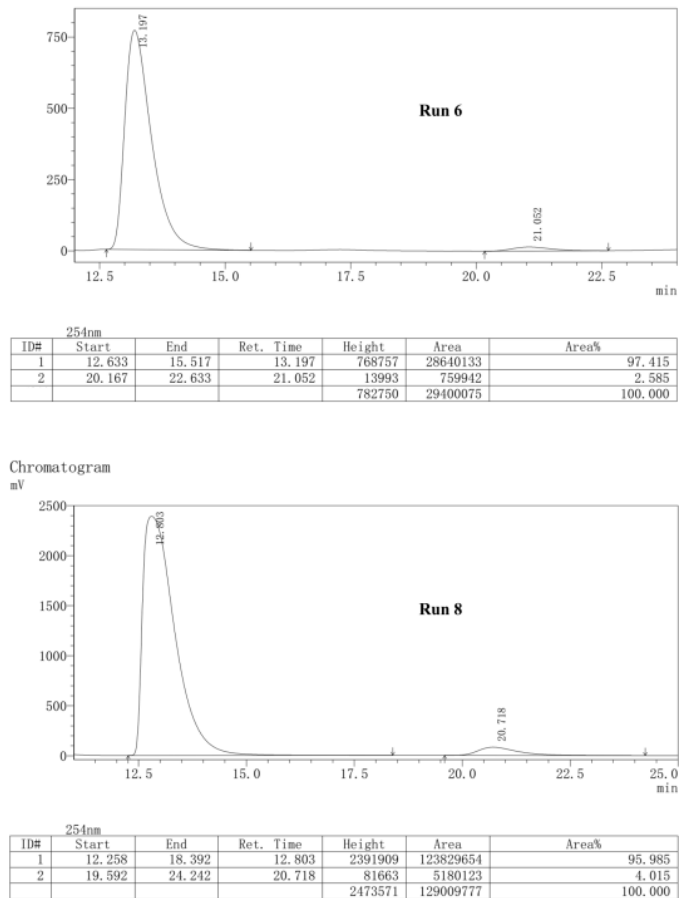

Chromatogran
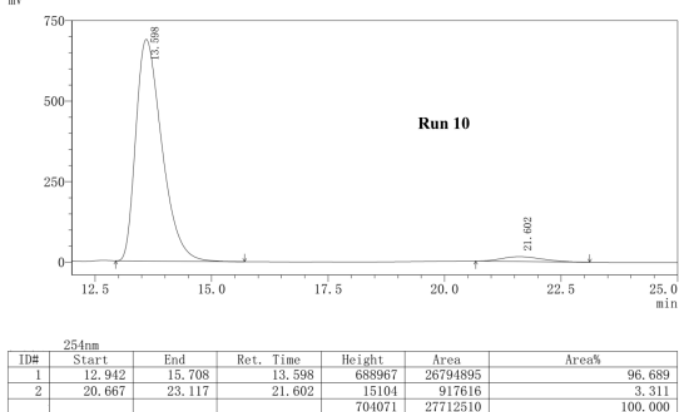

20.8 HPLC spectra of recycle experiment for Spiro-1 catalyzed Friedel-Crafts reaction of $\mathrm{N}$-Sulfonyl 4-bromoaldimine with indole 
$\underset{\mathrm{mV}}{\text { Chromatogram }}$

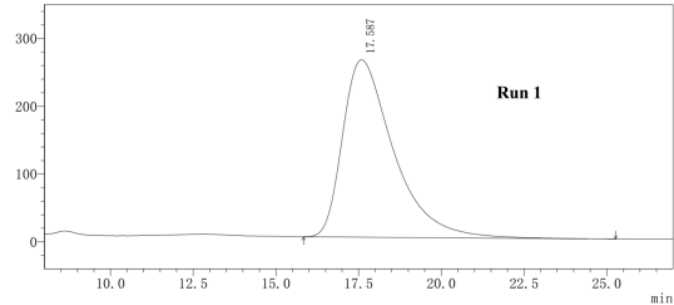

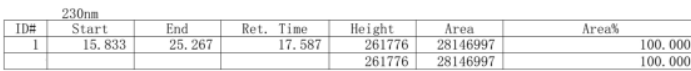
Chromatogram
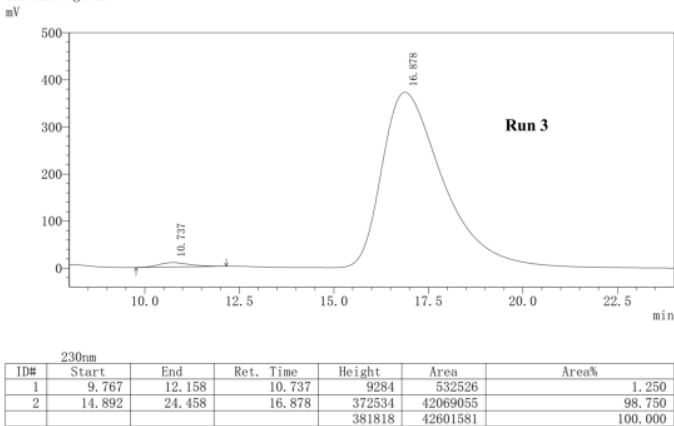
Chromatogram
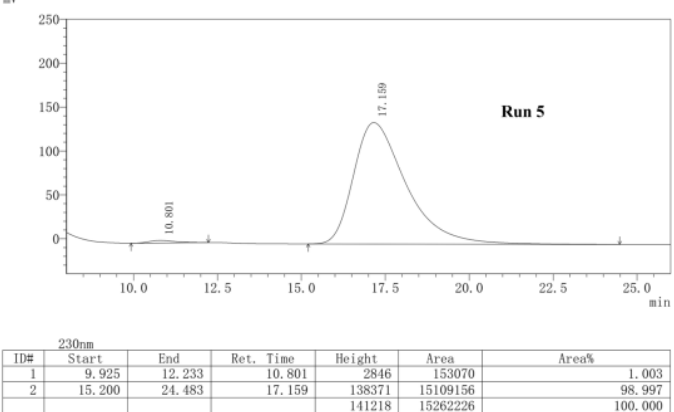

Chromatogram

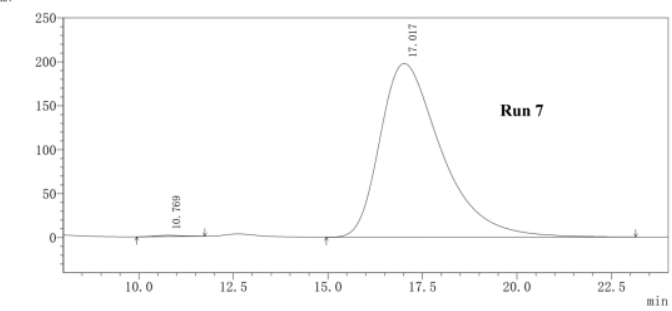

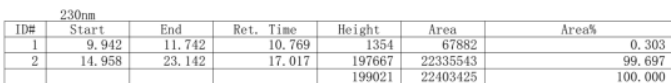

Chromatogram

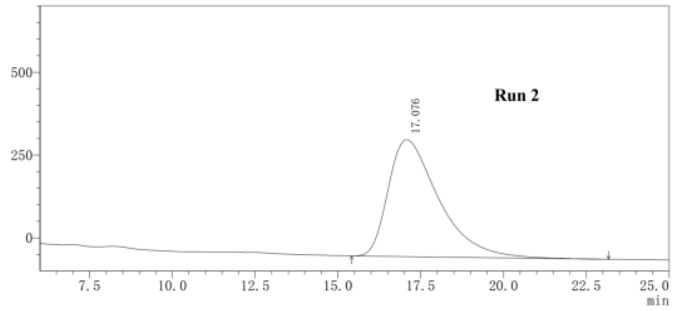

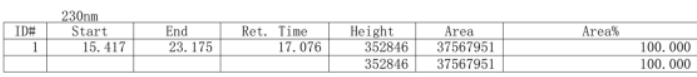

Chromatogran
my
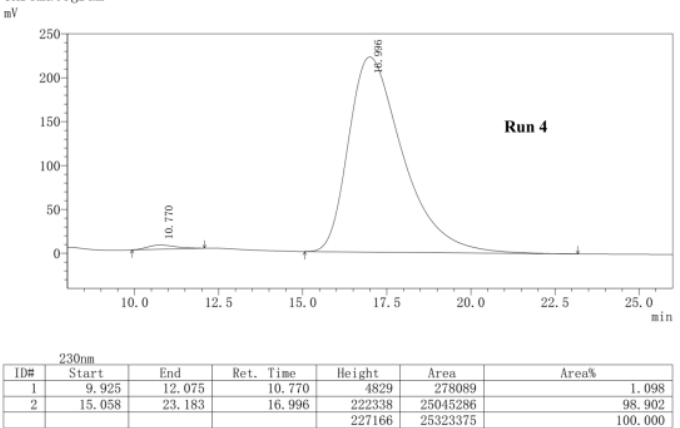

Chromatogram

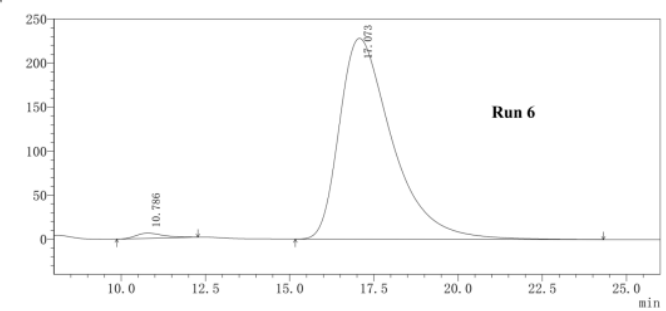

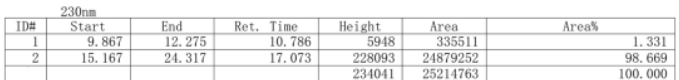

Chromatogram

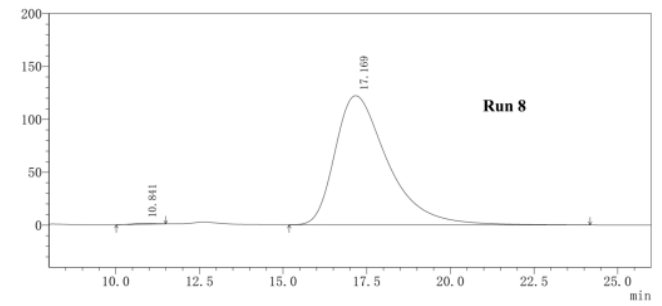

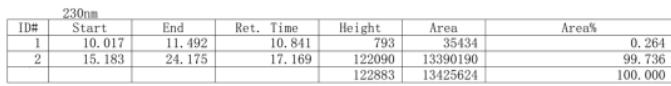


Chromatogram
$\mathrm{mV}$

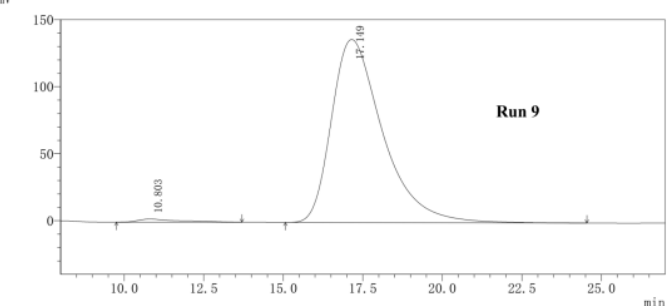

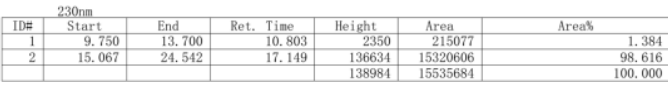

Chromatogram
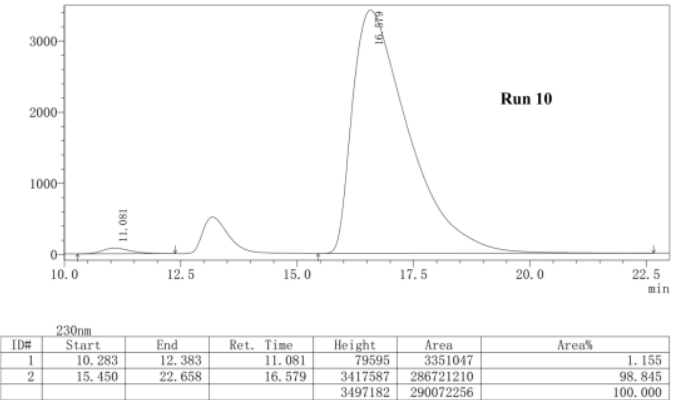

20.9 HPLC spectra of recycle experiment for Spiro-1 catalyzed iso-Pictet-Spengler Reaction of $o$-amino-benzylindole with Trifluoromethylated Ketone
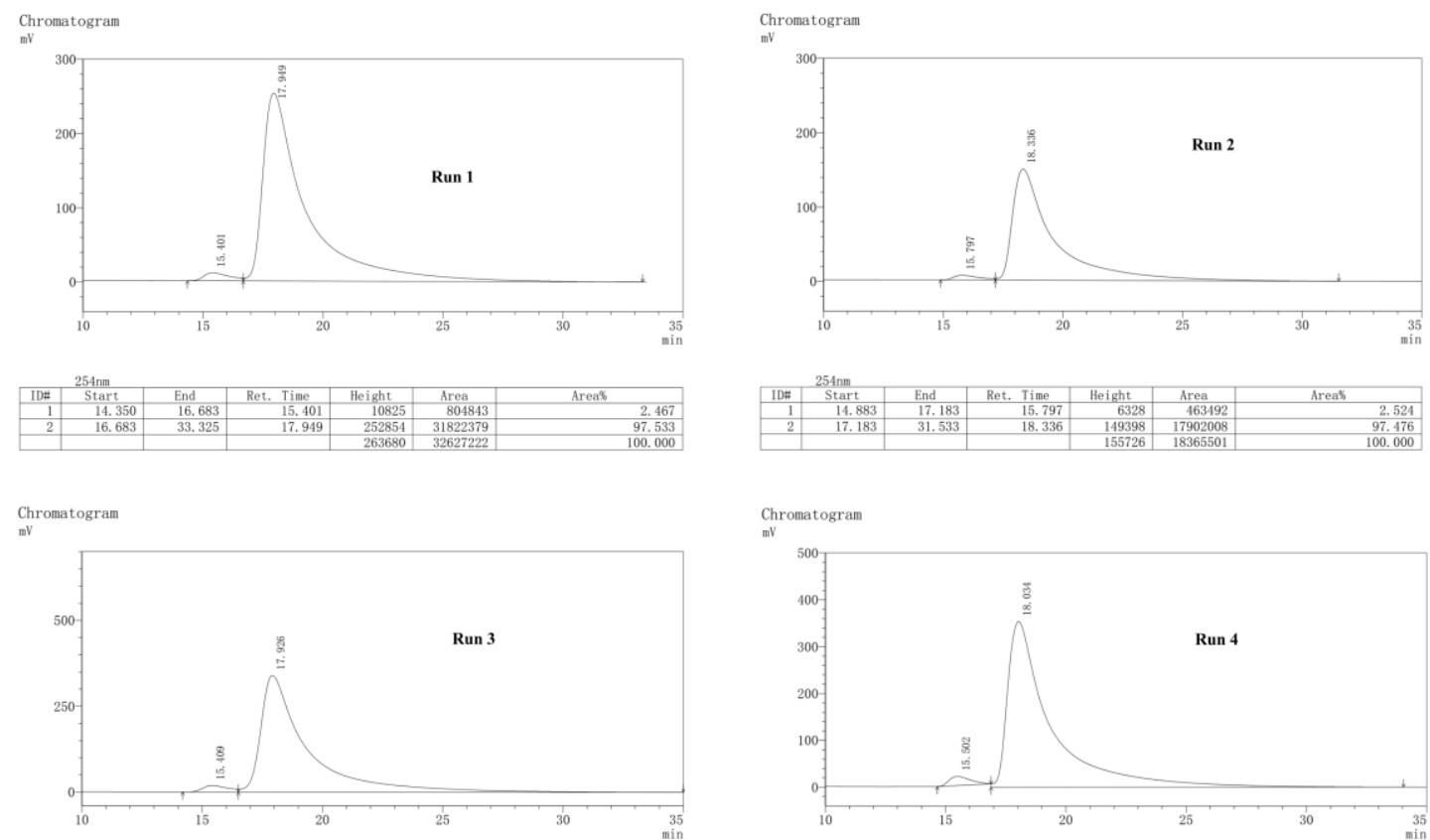

Chromatogram
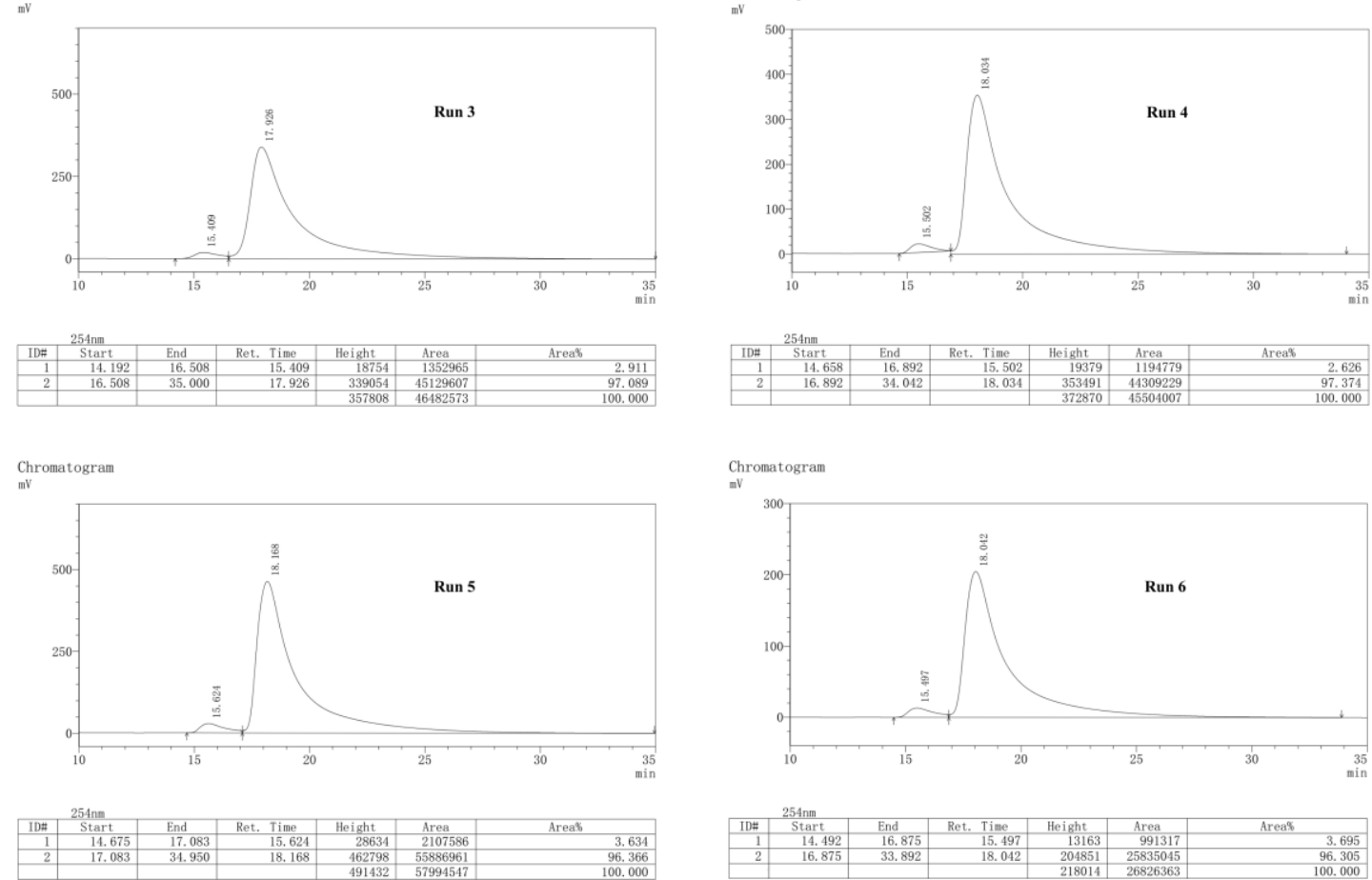

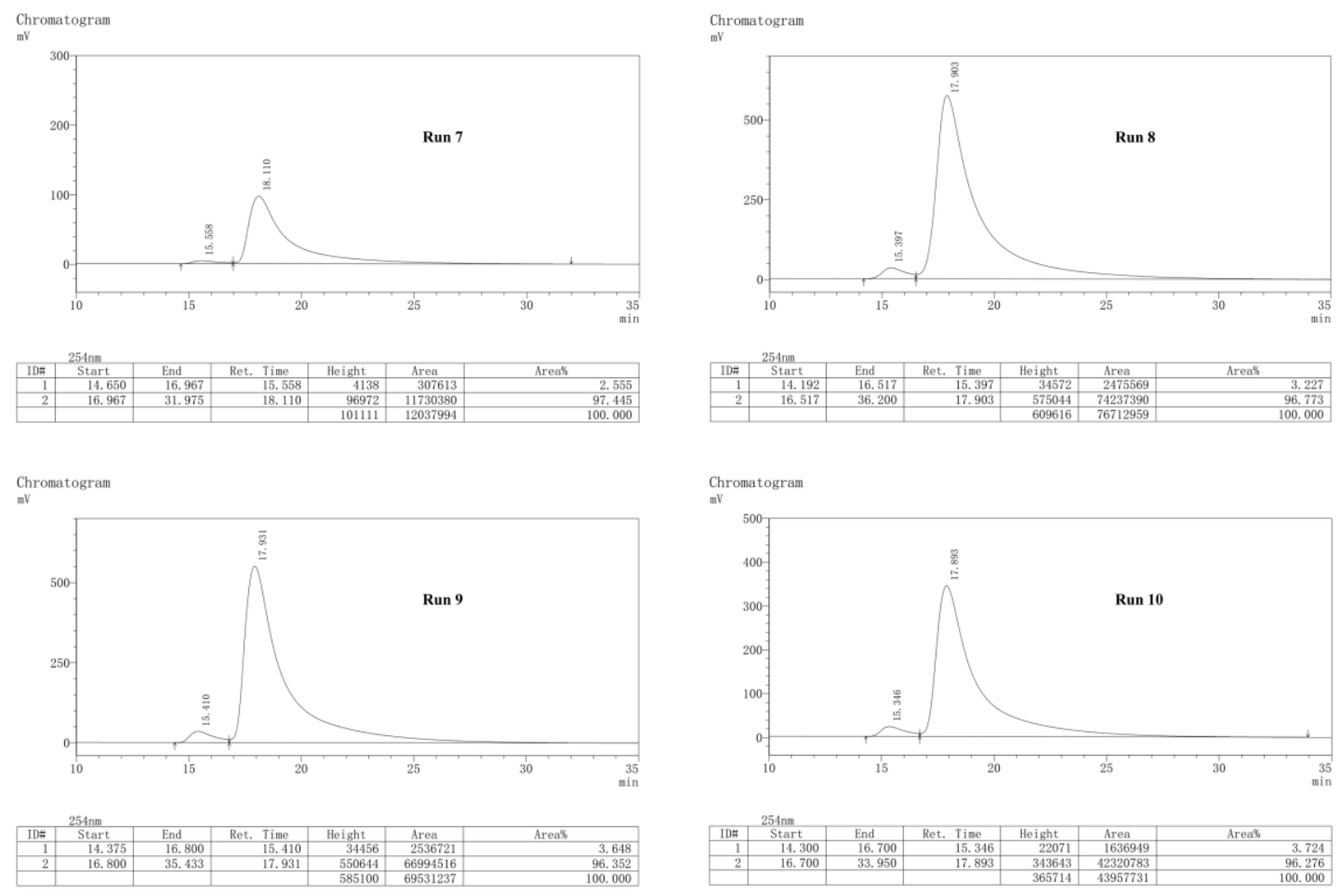

\section{REFERENCES}

(1) V. B. Birman.; A. L. Rheingold.; K. Lam. Tetrahedron: Asymmetry, 1999, 10, 125-131.

(2) J. H. Zhang.; J. Liao.; X. Cui.; Yu, K. B.; Zhu, J.; Deng, J. G.; Zhu, S. F.; Wang, L. X.; Zhou, Q.

L .; Chung, L. W.; Ye, T. Tetrahedron: Asymmetry, 2002,13, 1363-1366.

(3) Z. Y. Yang.; Y. X. Ni.; R. Liu.; Song, K. X.; Lin, S. H.; Pan, Q. M. Tetrahedron Letters, 2017, 58, 2034-2037.

(4) S. K. Sharma.; S. Sharma, P. K.; Agarwal, B. Kundu.; Eur. J. Org. Chem. 2009, 1309-1312. 